\title{
Defektabhängige Transporteigenschaften von Praseodym-Kalzium-Manganat
}

\author{
Dissertation \\ zur Erlangung des Doktorgrades \\ der Mathematisch-Naturwissenschaftlichen Fakultäten \\ der Georg-August-Universität zu Göttingen
}

vorgelegt von

Peter Moschkau

aus

Bad Salzungen

Göttingen 2009 
Referent:

Korreferent:
Prof. Dr. Christian Jooß

PD Dr. Vasily Moshnyaga

Tag der mündlichen Prüfung: 13.08 .2009 


\section{Inhaltsverzeichnis}

1 Einleitung 1

2 Grundlagen 3

2.1 Kristallstruktur . . . . . . . . . . . . . . 3

2.1.1 Struktur bei höheren Temperaturen . . . . . . . . . . . 3

2.1.2 Ordnungseinstellungen bei tiefen Temperaturen . . . . . 5

2.2 Defekte ......................... 6

2.2.1 Herstellungsbedingte Defekte . . . . . . . . . . . 6

2.2.2 Zwillingsbildung beim Wachstum auf STO . . . . . . . 7

2.2 .3 Vizinale Substrate . . . . . . . . . . . . . . 8

2.3 Polaronen . . . . . . . . . . . . . . . . . . . . . . . 9

2.3.1 Dynamik kleiner Polaronen für höhere Temperaturen . . . 11

2.3.2 Dynamik kleiner Polaronen bei tiefen Temperaturen . . . . 12

2.3.3 Dynamik großer Polaronen . . . . . . . . . . . . . . . . 12

2.4 Kolossale Widerstandseffekte in $\mathrm{PCMO}$. . . . . . . . . . . . 13

3 Ergebnisse $\quad 15$

3.1 Strukturuntersuchungen . . . . . . . . . . . . . . 17

3.1 .1 Oberflächenuntersuchungen . . . . . . . . . . 17

3.1.2 Spannungsanalyse durch Röntgenbeugung . . . . . . . . 18

3.1 .3 TEM Untersuchungen . . . . . . . . . . . . . . 21

3.1.4 SQUID Magnetisierungsmessungen . . . . . . . . . . 22

3.1.5 Interaktion an der Grenzfläche . . . . . . . . . . . . 25

3.2 Der Kolossale Magnet-Widerstands-Effekt . . . . . . . . . . . 26

3.3 Filme auf vizinalen Substraten . . . . . . . . . . . . . . 30 
3.4 Zusammenspiel B- und E-Feld . . . . . . . . . . . . . . . 33

3.4.1 Kinetik der Phasenübergänge . . . . . . . . . . . 33

3.4.2 Abhängigkeit des Widerstandes vom elektr. Feld . . . . . . 34

3.4.3 Elektrische Felder im Übergangsbereich . . . . . . . . . . 37

3.4.4 Remanenz der elektrisch leitfähigen Phase . . . . . . . . 39

3.4 .5 Vizinale Filme . . . . . . . . . . . . . . . . 44

4 Diskussion $\quad 47$

4.1 Transport in den unterschiedlichen Phasen . . . . . . . . 50

4.1 .1 ungeordnete Phase . . . . . . . . . . . . . 50

4.1.2 Ferromagnetisch-metallartige Phase . . . . . . . . 50

4.1 .3 Übergangsbereich . . . . . . . . . . . . . . . . . . . 52

4.2 Die Rolle der Defekte . . . . . . . . . . . . . . . . . . . . 54

4.2.1 Nachträgliche thermische Behandlung . . . . . . . . . . 54

4.2.2 Einfluss von Defekten auf die Aktivierungsenergie . . . . 58

4.3 CMR- und CER-Effekt . . . . . . . . . . . . . . 60

4.3 .1 Zustandsdiagramm . . . . . . . . . . . . 60

4.3.2 CMR- und CER-Effekt als konkurrierende Effekte . . . . . 62

4.3.3 Die elektrisch leitfähige Remanenz _ . . . . . . . . . . . 63

4.4 Vizinale Substrate . . . . . . . . . . . . . . . . . 64

4.5 Ladungsträger in der ferromagn.-metallart. Phase . . . . . . . . 67

$\begin{array}{lll}5 & \text { Zusammenfassung } & 71\end{array}$

$\begin{array}{ll}\text { A Experimentelles } & 73\end{array}$

A.1 Schichtherstellung . . . . . . . . . . . . . . . . 73

A.1.1 Gepulste Laserdeposition . . . . . . . . . . . . . . 73

A.1.2 Target . . . . . . . . . . . . . . . 74

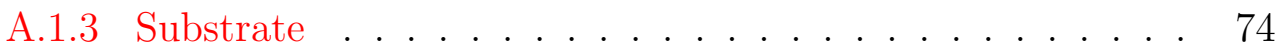

A.1.4 Strukturierung . . . . . . . . . . . . . . 75

A.1.5 Goldkontakte . . . . . . . . . . . . . . 76

A.2 Strukturelle Charakterisierung . . . . . . . . . . 76

A.2.1 Röntgendiffraktometrie . . . . . . . . . . . . 76

A.2.2 Oberflächenuntersuchung . . . . . . . . . . . . 78 
A.2.3 Magnetisierungsmessungen . . . . . . . . . . 78

A.3 Elektronische Charakterisierung . . . . . . . . . . . . . . . . 78

A.3.1 Messungen im elektrischen Feld . . . . . . . . . . . 78

A.3.2 Messungen im Magnetfeld . . . . . . . . . . . . . . 79

A.3.3 Zeitaufgelöste Widerstandsmessungen . . . . . . . . . . . 82

Literatur

Danksagung 


\section{Kapitel 1}

\section{Einleitung}

Hoch korrelierte Elektronensysteme sind durch eine enge Verknüpfung der Struktur mit den Transporteigenschaften gekennzeichnet. Das Einstellen von Ordnung, wie z. B. einer Ladungs-, Orbital oder Spinordnung, führt zu Phasenseparation, welche in den perowskitischen Manganaten $A_{1-x} B_{x} M_{n} O_{3}$ als Hauptmerkmal der komplexen Transporteigenschaften gedeutet werden [15]. Die Phasenseparation wird dabei auch als ,elektronsich" bezeichnet, da die involvierten Phasen eine identische chemische Zusammensetzung, eine ähnliche Struktur aber gravierende Unterschiede in den elektrischen Eigenschaften aufweisen.

Durch externe Stimulationen, wie z. B. dem Anlegen von elektrischen oder magnetischen Feldern, hydrostatischem Druck, Beleuchtung mit Licht oder Röntgenstrahlung, kann in diesen Materialien der Widerstand um viele Größenordnungen verändert werden [3, 14, 25, 36, 47, 63, 71, 72, 82, 83]. Die Mechanismen, die zu diesen Widerstandseffekten führen (z. B. kolossaler Magnetowiderstandseffekt (CMR-Effekt), kolossaler Elektrowiderstandseffekt (CER-Effekt)), sind nicht leicht zu verstehen und Gegenstand aktueller Forschung.

In dieser Arbeit wurde der Ladungsträgertransport an dem ladungs- und orbitalgeordneten System $\mathrm{Pr}_{0,68} \mathrm{Ca}_{0,32} \mathrm{MnO}_{3}$ (PCMO) untersucht [37], welches eine sehr starke Elektronen-Phononen-Kopplung zeigt. Aufgrund des niedrigen Toleranzfaktors von 0,94 [93] besitzt PCMO eine orthorhombische Kristallstruktur, wobei der Mn-O-Mn-Bindungswinkel mit $155^{\circ}$ in der ungeordneten Phase deutlich von dem ideal-kubischen von $180^{\circ}$ abweicht. Dies führt zu einer deutlichen Reduktion der elektronischen Bandbreite [67] bzw. zu einer Verringerung des Doppelaustausches im Sinne der Goodenough-Regeln [28, 52]. Nach [62, 61] sind die Ladungsträger in den Manganaten Polaronen. Dabei werden für Manganate mit kleinem Toleranzfaktor und starker Lokalisierung der Ladungen kleine Polaronenmodelle [34, 60,76], bzw. für Materialien mit großem Torleranzfaktor große Polaronenmodelle [34, 12] favorisiert. Da die Mobilität kleiner Polaronen durch thermisch aktivierte Hüpfprozesse bedingt ist, während große Polaronen einen bandartigen Transport zeigen [20], werden z. B. Übergänge von kleinen zu 
großen Polaronen im Magnetfeld als mögliche Ursache für den CMR diskutiert. Die Transporteigenschaften der Manganate hängen aber auch sensitiv von mechanischen Spannungen und kristallographischen Defekten ab. In PCMO Dünnfilmen reduzieren z. B. Zugspannungen das magnetische Feld, welches zum Aufschmelzen der geordneten Phase benötigt wird [73] und erhöhen die Ordnungstemperatur [94], wobei die Néel-Temperatur nahezu unberührt bleibt [50]. Ein ähnliches Verhalten wird für die Anwesenheit von Unordnung erwartet [60], welche z. B. durch Defekte in das System eingebracht wird.

In dieser Arbeit soll nun untersucht werden, wie sich die Existenz einer kristallinen Unordnung auf den Ladungsträgertransport auswirkt. Die Variation der Defektdichte geschieht hierbei über thermische Nachbehandlung von Dünnfilmen, die mittels gepulster Laserdeposition (PLD) hergestellt wurden. Des Weiteren werden über vizinale Substrate gezielt periodische Defekte eingebracht. In Anlehnung an das strukturell verwandte System der Hochtemperatursupraleiter $\mathrm{YBaCuO}$ werden mit der Herstellung von Filmen auf vizinalen Substraten wohlorientierte Antiphasengrenzen erwartet [32, 43].

In dem ersten Kapitel werden die physikalischen Grundlagen erarbeitet, welche für die Diskussion der Ergebnisse notwendig sind. Zunächst wird ein Überblick über die Kristallstruktur von PCMO gegeben, anschließend die Defekte im System vorgestellt und schließlich die Ladungsträger mit ihrer Dynamik dargelegt. Die Ergebnisse werden im Anschluss dargestellt. Den Anfang in diesem Kapitel machen die Strukturuntersuchungen, gefolgt von Transportmessungen zum kolossalen Magnet-Widerstands-Effekt, den Untersuchungen an vizinalen Substraten und letztlich dem Zusammenspiel von elektrischen und magnetischen Feldern sowie dem elektrischen Strom.

Für die Diskussion der Ergebnisse ist zu beachten, dass in der Literatur eine Kontroverse darüber besteht, ob die Ladungsordnung und der Ladungstransport im Bilde einer gemischten $\mathrm{Mn}^{3+} / \mathrm{Mn}^{4+}$-Valenz [31] oder durch Löcher in Sauerstoffähnlichen Zuständen (bindungszentrierte Ordnung der Zenerpolaronen) [17] bestimmt ist. In dieser Arbeit wird aufgrund der vorliegenden Ergebnisse [17, 42, 93] das Zener-Polaronen-Szenario favorisiert. Im Anhang werden die verwendeten experimentellen Techniken dargestellt. 


\section{Kapitel 2}

\section{Grundlagen}

In diesem Kapitel soll der für die Manganate typische enge Zusammenhang von Struktur, elektrischer Leitfähigkeit sowie magnetischer Ordnung am Beispiel des ladungsgeordneten, halbleitend bis isolierenden $\operatorname{Pr}_{1-x} \mathrm{Ca}_{x} \mathrm{MnO}_{3}$ Systems (PCMO) dargestellt werden. Dazu wird zunächst auf die Kristallstruktur bei hohen Temperaturen (oberhalb der Ladungsordnungstemperatur $T_{C O}$ ) und die Einstellung von Ordnungen bei tiefen Temperaturen (unterhalb der Ladungsordnungstemperatur $T_{C O}$ ) eingegangen. Der zweite Teil dieses Kapitels befasst sich mit der Frage der Defekte in dieser Struktur, insbesondere mit der Defektstruktur von epitaktischen PCMO-Schichten. Den Abschluss dieses Kapitels bilden die Darstellung der Transporteigenschaften im Rahmen von Polaronen-Modellen sowie die kolossalen Widerstandseffekte.

\subsection{Kristallstruktur}

\subsubsection{Struktur bei höheren Temperaturen}

In Abbildung 2.1 ist das Phasendiagramm für $\mathrm{Pr}_{1-x} \mathrm{Ca}_{x} \mathrm{MnO}_{3}$ über die KalziumDotierung $x$ in Anlehnung an Hill et al. [37] dargestellt. Die im gezeigten Phasendiagramm mit PI gekennzeichnete paramagnetisch isolierende Phase besitzt eine orthorombische Pnma Struktur. Ausgehend von einer idealen kubischen Perowskit-Einheitszelle erhält man im PCMO mit dem Toleranzfaktor von 0,94 [93] eine oktaedrische Verkippung, wie sie in Abbildung 2.2 skizziert ist. Diese Verkippungen führen zu einem Mn-O-Mn Bindungswinkel, der kleiner als $180^{\circ}$ ist. Die Reduzierung des Bindungswinkels hat eine starke Reduktion der Leitfähigkeit, wie auch dem damit verbundenen ferromagnetischen Doppel-Austausch zur Folge [27]. Die durch die Verkippung entstehende Einheitszelle ist, bezogen auf die kubische Einheitszelle, um $45^{\circ}$ in der a-b-Ebene gedreht und um näherungsweise einen Faktor $\sqrt{2}$ in jeweils a- und b-Richtung vergrößert. In c- 


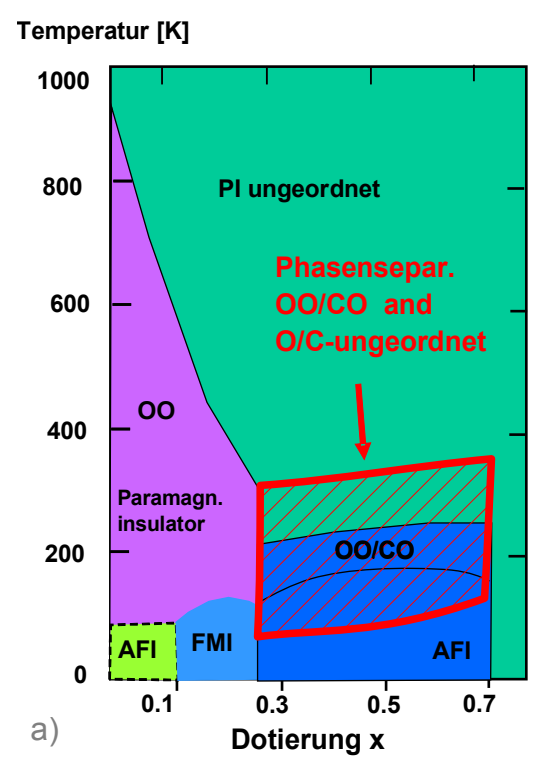

b)

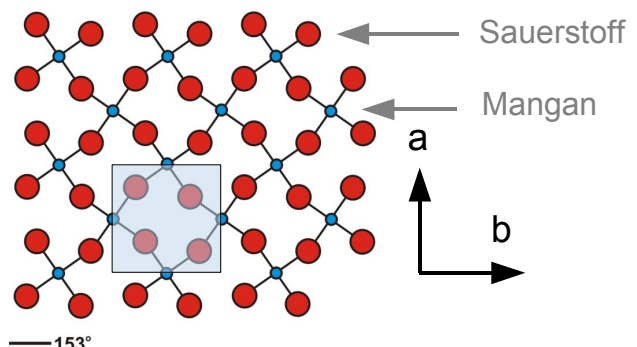

c)

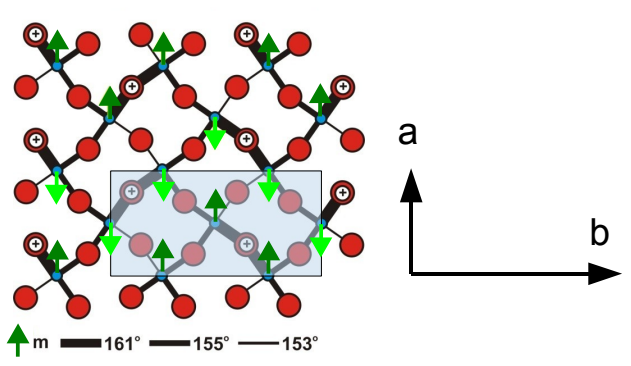

Abbildung 2.1: a) Phasendiagramm für $\mathrm{Pr}_{1-x} \mathrm{Ca}_{x} \mathrm{MnO}_{3}$; Idealisierung (ohne Praseodym/ Kalzium) als Netzwerk von $\mathrm{MnO}_{6}$ Oktaedern für die ungeordneten Phase Pnma b) und die $\mathrm{OO} / \mathrm{CO}$ Phase $P 2{ }_{1} m n$ c), mit eingezeichneter Einheitszelle jeweils in [001] Projektion

(verwendetet Abkürzungen: PI Paramagnetisch Isolierend, OO Orbitalordnung, CO Ladungsordnung, AFI Antiferromagnetisch Isolierend, FMI Ferromagnetisch Isolierend, O/C Orbital/Ladung).

Richtung kommt es etwa zu einer Verdopplung des Gitterparameters. Ein Übergang von der kubischen zur orthorombischen Struktur wird im PCMO, ähnlich wie bei anderen Manganaten (LCMO: [35], PSMO: [48]), bei Temperaturen von ca. $T=900^{\circ} \mathrm{C}$ erwartet. Durch die Kristallfeldaufspaltung kommt es zu einer Aufhebung der Entartung, welche aber für die Jahn-Teller-aktiven $\mathrm{Mn}^{3+}$ Ionen noch nicht komplett ist. Wie im Theorem des Jahn-Teller-Effektes [40] beschrieben, wird durch eine Verzerrung des Sauerstoffoktaeders diese Entartung aufgehoben. Dies geht mit einer Elongation des Sauerstoffoktaeders in z-Richtung bei einer gleichzeitigen Kompression in der $\mathrm{x}$ - und y-Richtung einher [15]. Die Dotierung des Praseodymmanganats mit Kalzium führt dazu, dass sich mobile Ladungsträger ausbilden (Polaronen). Die thermisch aktivierte Bewegung solcher dynamischen Jahn-Teller-Polaronen wird für den elektrischen Transport in der PI Phase verantwortlich gemacht [87]. 

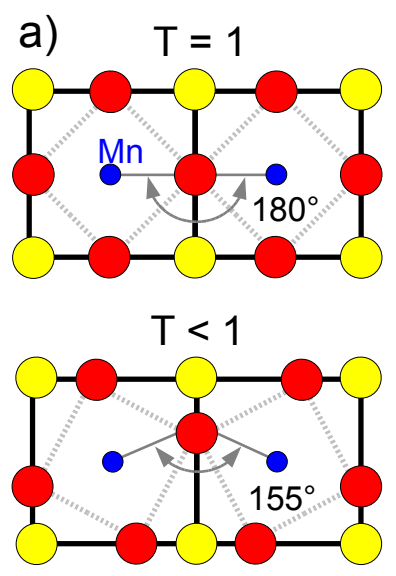

b)

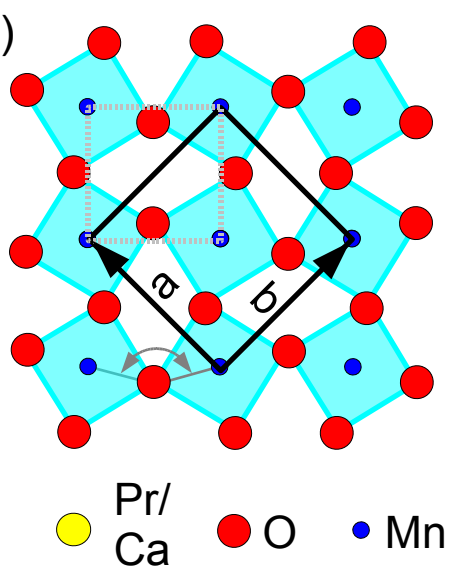

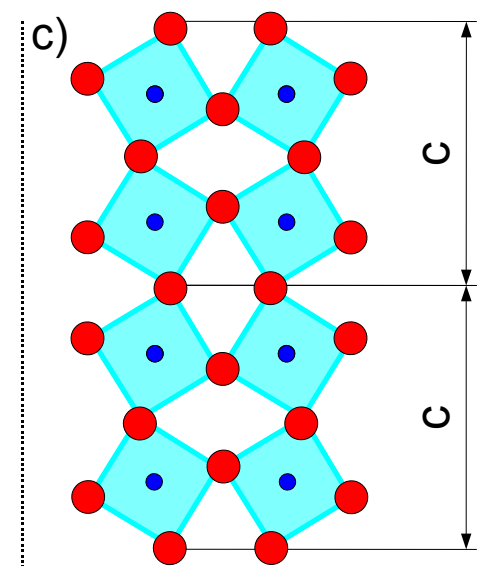

Abbildung 2.2: Schematische Darstellung der Verkippung der Sauerstoffoktaeder durch einen Toleranzfaktor $\neq 1$. a) Änderung des Mn-O-Mn Bindungswinkels, Veränderung der Einheitszelle in der a-b-Ebene (b) und entlang der dazu senkrechten c-Achse (c).

\subsubsection{Ordnungseinstellungen bei tiefen Temperaturen}

PCMO beschreitet bei ca. 230K (Ausgehend von einer 32\%-igen Kalziumdotierung, [31]) einen Phasenübergang in die Ladungs- und Orbitalgeordnete Phase $(\mathrm{OO} / \mathrm{CO})$. Die dynamischen Jahn-Teller-Verzerrungen gehen in statisch kooperative Verzerrungen über. Die resultierenden Gitterverzerrungen führen zu nichtgleichmäßigen Mn-O-Mn Bindungen (Abb. 2.1 c). Neben einfachen Typen von Jahn-Teller-Verzerrungen (uniaxiale Dehnungen und Stauchungen der $\mathrm{MnO}_{6}$ Oktaeder) kann es in Verbindung mit zusätzlichen Faktoren, wie einer Hybridisierung der 3d Orbitale von Mangan mit den 2p Orbitale vom Sauerstoff oder den 4f Orbitalen des Praseodyms, zu komplexeren Formen des Jahn-Teller Effektes kommen [85]. Die konkurrierenden Modelle (Manganplatz zentrierte Ladungsordnung gegenüber Mangan-Sauerstoff bindungszentrierte Ladungsordnung) hängen in subtiler Weise von den Details des Jahn-Teller Effektes ab [45], zum Beispiel nicht zentrosymetrischen Verzerrungen der $\mathrm{MnO}_{6}$ Oktaeder. Widesprüchliche Ergebnisse ([29, 31] versus [17, 42]) konnten erst in letzter Zeit durch eigene Beugungs- und Spektroskopieexperimente aufgeklärt werden [42]. Wesentlich hierfür ist die Beugung lokal in einzelnen Zwillingsdomänen, so war eine eindeutige Bestimmung der Raumgruppe $P 2{ }_{1} n m$ (entspricht einer bindungszentrierten Ladungsordung) möglich. Es kommt in PCMO zu einer Ladungsordnung, wobei die hinzudotierten Löcher bevorzugt an den Mn-O-Mn Bindungen mit den größten Bindungswinkeln lokalisieren. Paare von Jahn-Teller verzerrten ferromagnetisch gekoppelten $\mathrm{MnO}_{6}$ Oktaedern, verbunden durch diese Mn-O-Mn Bindungen mit starker Doppel-Ausstausch-Wechelwirkung [18, 99], formen dabei Zener- 
Polaronen [17]. Durch diese Ausrichtung der Orbitale zueinander kommt es neben der bindungszentrierten Ladungsordnung zu einer Orbitalordnung. Mit der Ausbildung der Zener-Polaronen kommt es zu einer Überstruktur durch Verdopplung der Einheitszelle vom PCMO entlang der b-Richtung. Der neue Gitterparameter dieser Überstruktur $b_{s}$ ist verglichen mit dem doppelten Gitterparameter $b_{0}$ der Pnma Struktur $\frac{b_{s}}{2 \cdot b_{0}}=0,983$. Dies impliziert eine deutliche Änderung des spezifischen Volumens, somit handelt es sich beim Übergang von der Pnma in die $P 2{ }_{1} m n$ Phase um einen Phasenübergang 1. Ordnung. Die Ordnungseinstellung erstreckt sich über einen Temperaturbereich von $300 \mathrm{~K}$ bis zu $80 \mathrm{~K}$ [93], wobei beide Phasen koexistieren (Phasenseparation). Unterhalb der Néel-Temperatur (140 K, [41]) tritt zusätzlich die antiferromagnetische Ordnung (CE-Typ) der Mn-O-Mn Dimere ein (Abb. 2.1 c) [17]. Diese ist mit einer zusätzlichen Verdopplung der Einheitszelle in a-Richtung verbunden.

\section{$2.2 \quad$ Defekte}

\subsubsection{Herstellungsbedingte Defekte}

Die in dieser Arbeit verwendeten Dünnfilmproben wurden mittels gepulster Laserdeposition (kurz PLD, siehe Anhang A.1.1) hergestellt. Des Weiteren wurden Proben untersucht, welche durch ein Ionenstrahlsputter-Verfahren hergestellt wurden. Bei der PLD wird das vom Target ablatierte Material abhängig vom
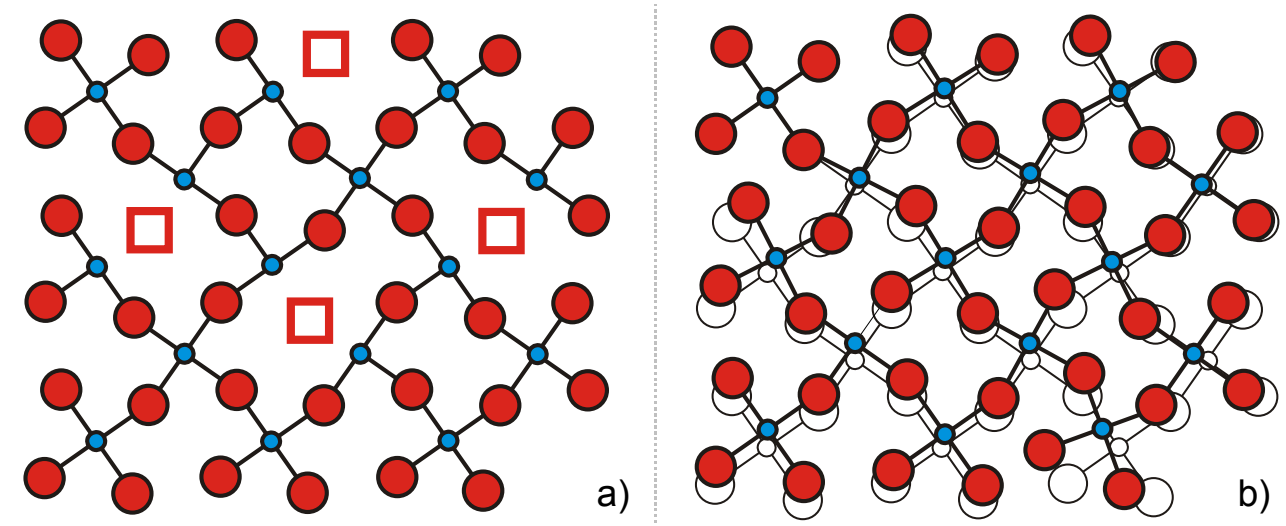

Abbildung 2.3: Herstellungsbedingte Defekte. a) Sauerstoffleerstellen, b) Fehler im oktaedrischen Kippsystem.

Sauerstoffdruck mit Energien von bis zu 100 eV auf dem Substrat deponiert. Bei dem verwendeten Sauerstoffdruck von 0,2 mbar treten typische Energien im eV 
Bereich auf. In den bereits deponierten Schichten kann es dadurch zum Beispiel zu Fehlern im oktaedrischen Kippsystem kommen (Abb. 2.3 b), [90]. Aufgrund der relativ hohen Depositionstemperaturen sollten diese Defekte aber bereits während der Herstellung größtenteils wieder ausheilen und somit keine große Rolle spielen. Ein ähnliches Verhalten findet man bei den Proben, die mittels Sputtern hergestellt wurden, wobei hier die Energien, mit denen das ablatierte Material auf dem Substrat ankommt, minimal höher liegen können. Eine weitere Möglichkeit für herstellungsbedingte Defekte sind Sauerstoffleerstellen (Abb. 2.3 a). Diese haben sehr starken Einfluss auf das Transportverhalten und den Magnetismus von Manganaten [4]. Durch die Gitterfehlpassungen zum Substrat entstehen im PCMO-Dünnfilm zudem mechanische Spannungen, die ebenfalls einen deutlichen Einfluss auf die Struktur und die Eigenschaften von Manganaten haben [13, 74, 94, 98]. Partiell können die Spannungen über Versetzungen auch wieder abgebaut werden $[26,30]$.

\subsubsection{Zwillingsbildung beim Wachstum auf STO}

Für diese Arbeit wurden PCMO-Dünnfilme auf dem Substrat Strontiumtitanat $\mathrm{SrTiO}_{3}$ (im Folgenden kurz STO) abgeschieden. PCMO kann auf STO in insgesamt 6 Zwillingsorientierungen aufwachsen (Abb. 2.4 c). Ausgehend von (001)-
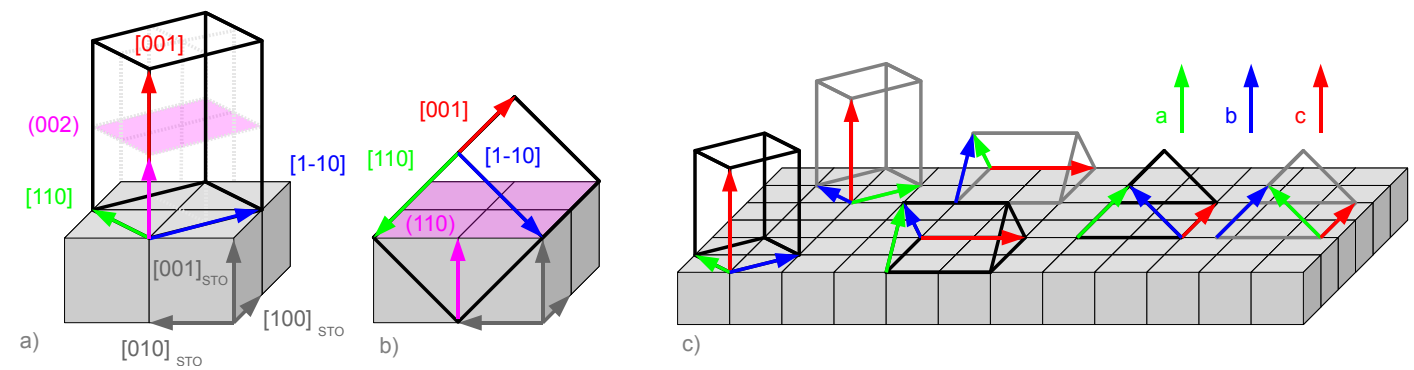

Abbildung 2.4: Mögliche Zwillingsorientierungen in PCMO auf STO, a) c-Achse „outof-plane“, b) c-Achse „in-plane“, c) Gesamtübersicht schematisch.

orientiertem STO kann PCMO zum einen mit der c-Achse ([001]PCMO) parallel zur [001]-Richtung von STO kristallisieren. Die beiden möglichen Epitaxiebeziehungen hierzu haben eine in-plane-Orientierung von PCMO[110][1-10]//STO[100][010] bzw. PCMO[1-10][110]//STO[100][010]. Zum Anderen kann die cAchse in der Ebene orientiert sein. Diese vier Zwillinge haben eine in-plane Orientierung von PCMO[-110][001]//STO[100][010] bzw. PCMO[001][1-10]//STO[100] [010] sowie PCMO[110][001]//STO[100][010] bzw. PCMO[001][-1-10]//STO[100] [010]. Die Wahrscheinlichkeit mit der die Zwillinge kristallisieren, scheint von 
der Kalziumdotierung abzuhängen. Für eine Dotierung von 30\% Kalzium ist mit einem Wachstum einer in-plane c-Achse zu rechnen [26], wohingegen für eine Dotierung von 50\% eher eine c-Achse senkrecht zum Substrat erwartet wird [75]. Unter Berücksichtigung der Einkristall-Werte der Einheitszelle für PCMO (Tabelle 2.1) im Verhältnis zu STO ergibt sich eine Gitterfehlpassung von 1,7\% für die c-Achse, wohingegen die Diagonale der a-b-Achse eine Gitterfehlpassung von 1,4\% hat. Daher ist für die vorliegende Kalziumdotierung ein Wachstum mit der c-Achse senkrecht zur Substratebene (out-of-plane) günstiger.

\begin{tabular}{rr|cc|c} 
STO & a-Achse & 3,905 & $\AA$ & \\
& Diagonale $\overline{a a}$ & 5,522 & $\AA$ & \\
$2 \cdot$ a-Achse & 7,809 & $\AA$ & Fehlpassung [\%] \\
\hline PCMO & a-Achse & 5,423 & $\AA$ & 1,8 \\
& b-Achse & 5,469 & $\AA$ & 1,0 \\
Diagonale $\overline{a b}$ & 7,702 & $\AA$ & 1,4 \\
c-Achse & 7,676 & $\AA$ & 1,7
\end{tabular}

Tabelle 2.1: Gitterparameter für die STO- und PCMO-Einheitszelle, die Fehlpassungen für $P C M O_{a-A c h s e}$ und $P C M O_{b-A c h s e}$ beziehen sich auf $S T O_{\overline{a a}}$, die anderen beiden Fehlpassungen von PCMO auf die doppelte Einheitszelle von STO. Daten für eine Dotierung von $\mathrm{x}=0,32$ (gemittelt, nach [41]).

\subsubsection{Vizinale Substrate}

Neben den zuvor beschriebenen Defekten wurden durch die Nutzung von vizinalen Substraten gewollt periodisch angeordnete Defekte eingebracht. An den Stufenkanten kann es zur Bildung von Anti-Phasen-Grenzen im Kippsystem der Oktaeder kommen, insofern sich die Systematik der Verkippungen der Sauerstoffoktaeder nicht über die Stufen fortsetzt. Dies ist in Abbildung 2.5 a) schematisch dargestellt und in c) als TEM-Bild an einer Probe aus dieser Arbeit zu sehen. Wie später anhand der Messdaten gezeigt wird, kommt es für PCMO auf vizinalen Substraten zusätzlich zu einer anisotropen Verspannung, ähnlich wie in Hochtemperatur-Supraleitern (HTSL) [7], was in Abbildung 2.5 b) skizziert ist. 


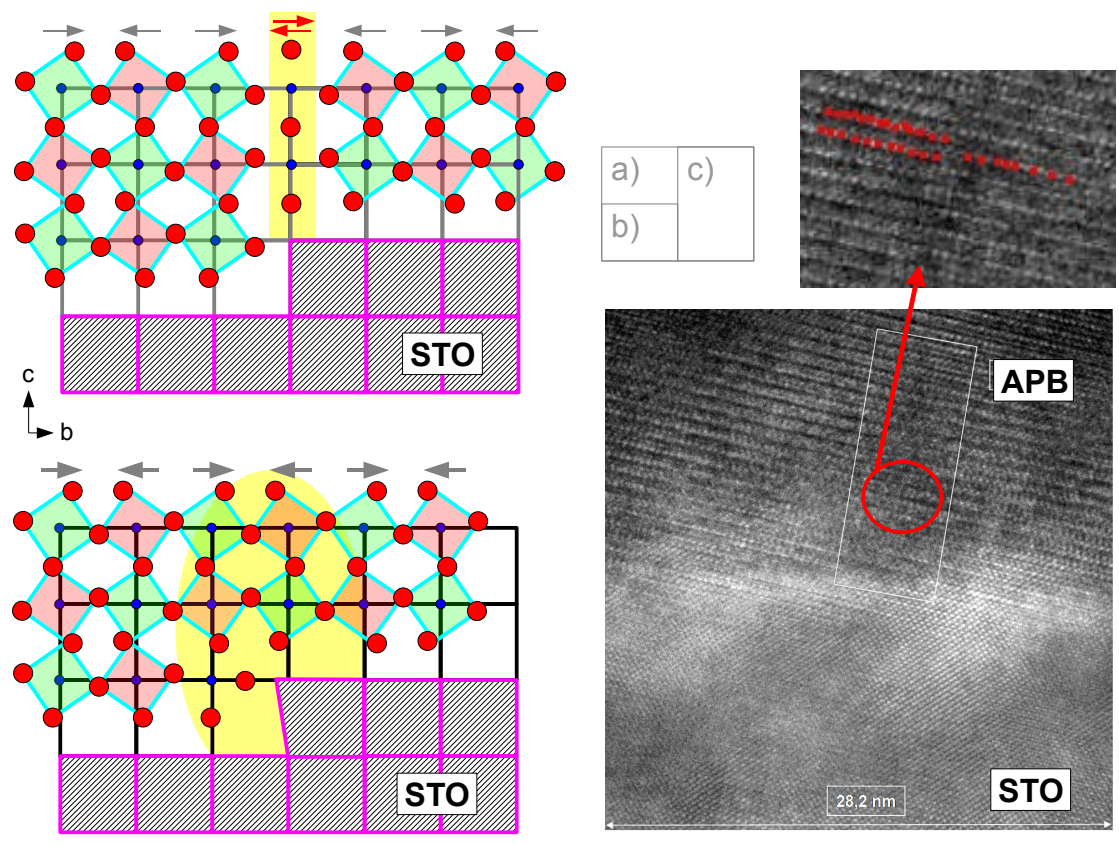

Abbildung 2.5: epitaktisch auf STO gewachsenes PCMO mit gezielt eingebrachten periodischen Defekten. a) Anti-Phasen-Grenze (APB) im Kippsystem, b) Ausbildung von Spannungen entlang der Stufenkanten, c) cross-section TEM-Bild einer APB.

\subsection{Polaronen}

Polaronen sind im einfachsten Bild Ladungsträger, die das umgebende Gitter polarisieren und verzerren. Zur näheren Betrachtung ist im Folgenden der „Holstein" -Hamilton-Operator [38] dargestellt. Er gibt ein an einen harmonischen Oszillator gekoppeltes Elektron wieder.

$$
H=\underbrace{+\hbar \omega \cdot \sum_{i}\left(a_{i}^{+} a_{i}+\frac{1}{2}\right)}_{\text {Term } 1} \underbrace{-t \cdot \sum_{i, k(N N)}\left(c_{i \sigma}^{+} c_{k \sigma}+c_{k \sigma}^{+} c_{i \sigma}\right)}_{\text {Term } 2} \underbrace{-g \cdot \sum_{i, \sigma} c_{i \sigma}^{+} c_{i \sigma}\left(a_{i}^{+}+a_{i}\right)}_{\text {Term } 3}
$$

Der erste Term beschreibt die Generierung bzw. Vernichtung eines Phonons mit der Energie $\hbar \omega$ am $i$-ten Gitterplatz. Der zweite Term bezieht sich auf die Elektronen und spiegelt die Reduktion der kinetischen Energie durch Platzwechselvorgänge nächster Nachbarn wieder, wobei t das Transferintegral und $c_{i \sigma}^{+}$und $c_{k \sigma}^{+}$die Erzeugungs- bzw. ohne + die Vernichtungsoperatoren der Elektronen am Platz $i$ bzw. $k$ darstellen. Der dritte Term beschreibt die Elektronen-Phononen- 
Kopplung, mit der Kopplungsenergie $g$. Damit ist die Bindungsenergie eines Polarons $J$ festgelegt:

$$
J \equiv \frac{g^{2}}{\hbar \omega}
$$

Für die Beschreibung von unterschiedlichen Arten von Polaronen sind die im Folgenden aufgeführten Kopplungskonstanten wichtig. Ihre Interpretation nach Feinberg [23] ist hinzugefügt.

$$
\begin{aligned}
& A \equiv \frac{\hbar \omega}{t}= \begin{cases}\ll 1 & \text { adiabatisch: das Elektron folgt den Ionen } \\
\gg 1 & \text { anti-adiabatisch: die Ionen folgen dem Elektron }\end{cases} \\
& \alpha \equiv \frac{g}{\hbar \omega}= \begin{cases}\ll 1 & \text { single-phonon: schwaches Polaron } \\
\gg 1 & \text { multi-phonon: starkes Polaron }\end{cases} \\
& \lambda \equiv \frac{J}{t}= \begin{cases}\ll 1 & \text { schwache Lokalisierung: großes Polaron } \\
\gg 1 & \text { starke Lokalisierung: kleines Polaron }\end{cases}
\end{aligned}
$$

Mit dem Wissen der im System auftretenden Energien und diesen Kopplungskonstanten ist eine Klassifizierung der Polaronen in verschiedene Regime möglich. Eine vereinfachte Skizze für zwei näher untersuchte Kombinationen ist in Abbildung 2.6 dargestellt: In a) ist ein stark lokalisiertes Polaron gezeigt $(\lambda \gg$
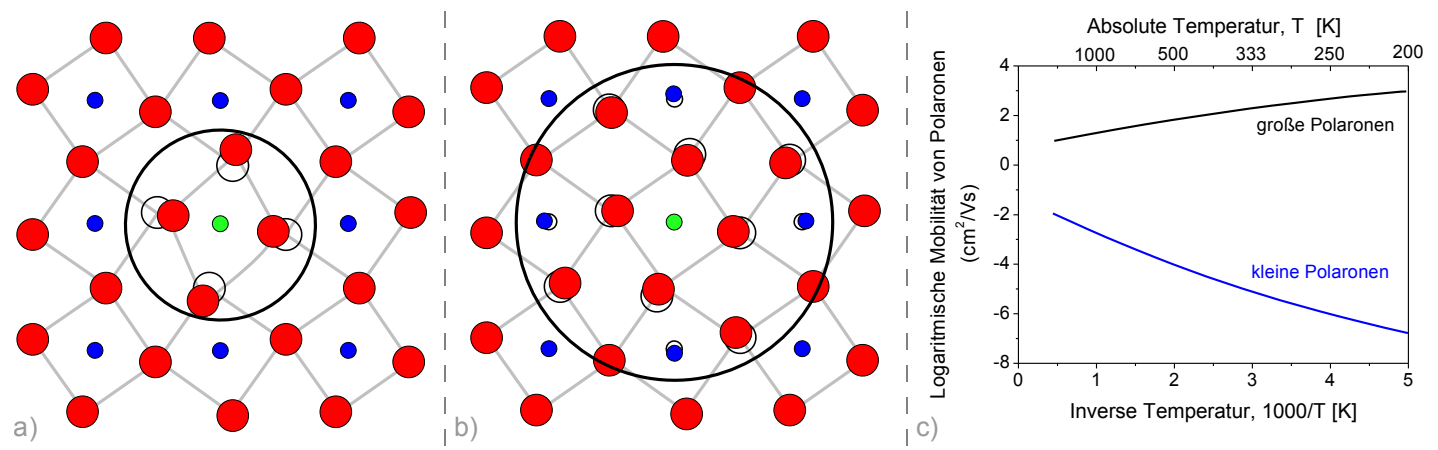

Abbildung 2.6: a) starkes kleines Polaron, b) schwaches großes Polaron, c) Mobilität der Polaronen nach [20].

$1 ; \alpha \gg 1)$. Es verzerrt das Gitter in naher Umgebung stark und ist in seinem eigenen Verzerrungsfeld gefangen. Das schwach lokalisierte große Polaron (Abb. $2.6 \mathrm{~b} ; \lambda \ll 1 ; \alpha \ll 1)$ ) hingegen beeinflusst die Ionen in einem viel größeren Gitterbereich. Dies hat großen Einfluss auf die Mobilität, wie in den folgenden Abschnitten beschrieben. 


\subsubsection{Dynamik kleiner Polaronen für höhere Temperatu- ren}

Kleine Polaronen bewegen sich für höhere Temperaturen $\left(T \geq \frac{\Theta_{D}}{2} ; \Theta_{D}\right.$ : DebyeTemperatur) mit ihrer Gitterverzerrung durch thermisch aktivierte Hüpfprozesse. Dafür benötigen sie die Aktivierungsenergie $E_{a}$, welche der halben Polaronenbildungsenergie entspricht. Ausgehend von einer Sprungweite a der Polaronen, erhält man die Diffusionskonstante $D(T)=\frac{a^{2}}{2 \pi} \cdot \omega_{0} \cdot \exp \left(-\frac{E_{a}}{k_{B} T}\right)$. Hierbei ist $\omega_{0}$ die Phononenfrequenz, $T$ die Temperatur und $k_{B}$ die Boltzmann-Konstante. Die Mobilität $\mu$ erhält man über die Einstein-Gleichung $\mu(T)=\frac{e \cdot D(T)}{k_{B} \cdot T}$. Dabei steht $e$ für die Elementarladung. Die Mobilität kleiner Polaronen nimmt mit abnehmender Temperatur ab (siehe auch Abb.2.6 c). Über die Definition der Leitfähigkeit $\sigma=n \cdot e \cdot \mu(T)$ mit der Anzahl der Ladungsträger $n$ erhält man das ThermischAktivierte-Polaronen Model (kurz TAP-Model) [8]. Dies wird im Folgenden unter Einbeziehung eines elektrischen Feldes $E=\frac{V_{D S}}{L}$ (angelegte Spannung durch Abstand) dargestellt:

$$
\frac{I}{A}=j=n e a \frac{\omega_{o p}}{\pi} \sinh \left(\frac{e V_{D S} a}{2 k_{B} T L}\right) \exp \left(-\frac{E_{a}}{k_{B} T}\right)
$$

Hierbei stehen $I$ für den Strom, $A$ für die stromdurchflossene Fläche sowie $j$ für die resultierende Stromdichte. Betrachtet man die Anregung nur für kleine elektrische Felder, so kann man mit der Vereinfachung $\sinh (x) \approx x$ die Formel umschreiben in:

$$
j=n e^{2} a^{2} \frac{\omega_{o p}}{\pi} \frac{V_{D S}}{2 k_{B} T L} \exp \left(-\frac{E_{a}}{k_{B} T}\right) .
$$

Dies gilt jedoch nur bis zu einem kritischen elektrischen Feld $E_{c}$. Im adiabatischen Limit ist der Zusammenhang zwischen der Leitfähigkeit und dem elektrischen Feld für das Hüpfen von Polaronen wie folgt gegeben [80]:

$$
\frac{\sigma(E, T)}{\sigma_{0}(T)}=\frac{E_{c}(T)}{E} \cdot \sinh \left(\frac{E}{E_{c}(T)}\right)
$$

Über eine Arrhenius-Auftragung des vereinfachten TAP-Modells kann man die Aktivierungsenergie direkt aus der Steigung bestimmen.

$$
\ln \frac{\rho}{T}=\frac{E_{a}}{k_{B} T}+\ln \frac{2 k_{B} \pi}{n e^{2} a^{2} \omega_{o p}} .
$$

Das TAP-Model ist nur oberhalb der halben Debye-Temperatur $\frac{1}{2} \Theta_{D}$ gültig. Für die verwendete Zusammensetzung vom PCMO liegt $\Theta_{D}$ bei 324-344 K [10, 33]. 


\subsubsection{Dynamik kleiner Polaronen bei tiefen Temperatu- ren}

Bislang wurde die Mobilität der Polaronen unter Vernachlässigung der Tunnelprozesse betrachtet. Bei tiefen Temperaturen $\left(T \leq \frac{\Theta_{D}}{2}\right)$, wenn keine Hüpfprozesse mehr möglich sind, setzt ein bandartiger Transport ein. Nach der Theorie von Lang und Firsov verhält sich der Widerstand für Temperaturen $T$ kleiner als $T<\frac{2 t_{p}}{k_{B}}$, wobei $t_{p}$ für das Hopping-Integral des Polarons steht, wie folgt [53]:

$$
\rho=\rho_{0}+\frac{C_{M} \omega_{s}}{\sinh ^{2}\left(\frac{\hbar \omega_{s}}{2 k_{B} T}\right)}
$$

Hierbei steht $\omega_{s}$ für die gemittelten Frequenzen der Soft-Moden. Es kommen nur die tiefstliegenden optischen Moden in Frage. Im verwendeten System gehören diese zur Verkippung des Sauerstoffoktaeder, welche stark an die Ladungsträger gebunden sind $[1,19]$. $C_{M}$ ist konstant und proportional zur effektiven Masse des Polarons.

\subsubsection{Dynamik großer Polaronen}

Große Polaronen sind dadurch bestimmt, dass ihre kinetische Energie größer ist als ihre Bindungsenergie $(\lambda \ll 1)$. Im Gegensatz zu den kleinen Polaronen benötigen sie keine Aktivierungsenergie. Der Widerstand großer Polaronen ist durch die Streuung an den Gitterschwingungen bestimmt, insofern man die Streuung an Defekten vernachlässigt. Da mit abnehmender Temperatur auch die Gitterschwingungen nachlassen, steigt die Mobilität der großen Polaronen (siehe auch hierfür Abb. 2.6 c) [20].

Nach einem Modell von Feynman [24] gilt für die Mobilität:

$$
\mu=\frac{e}{2 m \cdot \omega_{L O} \alpha} \frac{2}{3}\left(\frac{w}{v}\right)^{3} \exp \left(\frac{v^{2}-w^{2}}{w^{2} v}\right) e^{\hbar \omega_{L O} / k T} \frac{k T}{\hbar \omega_{L O}} .
$$

Dabei sind $v$ und $w$ Funktionen der Kopplungskonstanten $\alpha$, welche sich aus dem Feynman-Polaronen-Modell ableiten. 


\subsection{Kolossale Widerstandseffekte in PCMO}

PCMO zeigt sowohl über den gesamten Dotierbereich von Kalzium wie auch über den gesamten beobachtbaren Temperaturbereich ohne magnetisches bzw. elektrisches Feld isolierendes Verhalten, wobei oberhalb der Temperatur der Orbitalund Ladungsordnung $T_{C O / O O}$ (kurz Ordnungstemperatur) die ungeordnete Phase Pbnm vorherrscht und diese unterhalb der Ordnungstemperatur in die ZenerPolaronen geordnete Phase $P 2_{1} n m$ übergeht (Abb. 2.8) ${ }^{1}$. Je nach Temperatur ${ }^{2}$ und Magnetfeld kann die ZP-geordnete Phase in die ferromagnetisch-metallartige Phase überführt werden, wobei Widerstandsänderungen um mehr als 10 GröBenordnungen beobachtet wurden. Für die Dotierungen $\mathrm{x}=0,3$ sowie $\mathrm{x}=0,35$
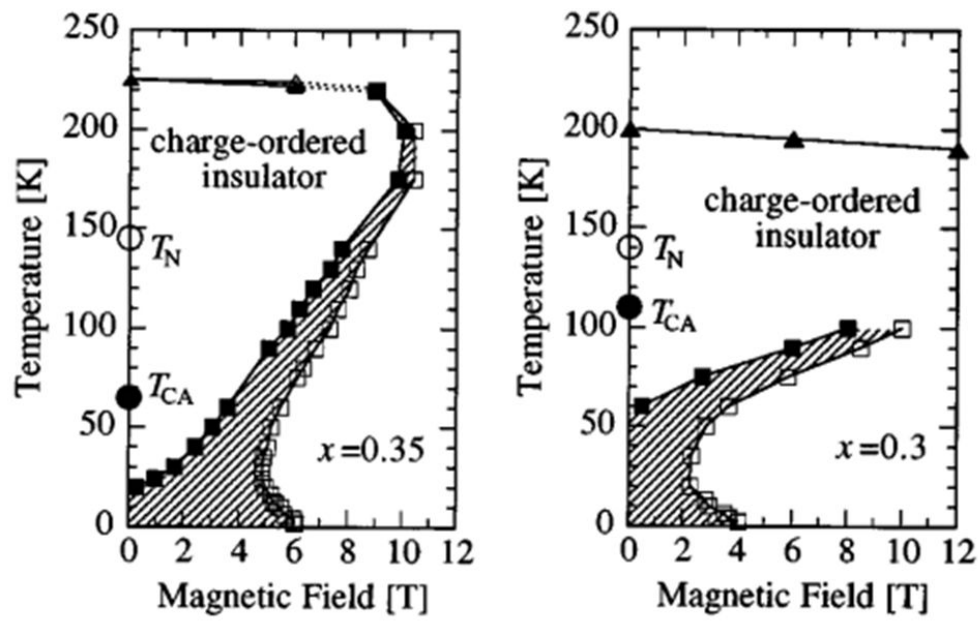

Abbildung 2.7: Phasendiagramm Temperatur über Magnetfeld nach [83]; PCMO, $\mathrm{x}=0,3$ (rechts), $\mathrm{x}=0,35$ (links).

ist dies in Abb. 2.7 dargestellt [83]. Erniedrigt man, aus der ferromagnetischmetallartigen Phase kommend, isotherm das Magnetfeld, so kommt es erst für niedrigere Magnetfelder zum Übergang zurück in die ZP-geordnete Phase, wodurch eine Hysterese entsteht, welche durch den schraffierten Bereich gekennzeichnet ist. Für sehr niedrige Temperaturen verbleibt man im Phasendiagramm im schraffierten Bereich. Die Remanenz für den elektrisch leitfähigen Zustand liegt für eine Dotierung von $\mathrm{x}=0,32 \mathrm{bis}$ zu einer Temperatur von $35 \mathrm{~K}$ vor (Abschnitt 3.4.4). Des Weiteren sind in Abb. 2.7 die Néel-Temperaturen $\left(T_{N}\right)$ sowie die Temperatur, ab der ein Übergang in den verkanteten Antiferromagnetismus $\left(T_{C A}\right)$ erwartet wird, mit eingezeichnet.

\footnotetext{
${ }^{1}$ Auf den Übergang im Nullfeld unterhalb von $45 \mathrm{~K}$ wird in der Diskussion eingegangen.

${ }^{2}$ Metall-Isolator Übergangstemperatur im Folgenden kurz $T_{M I T}$
} 


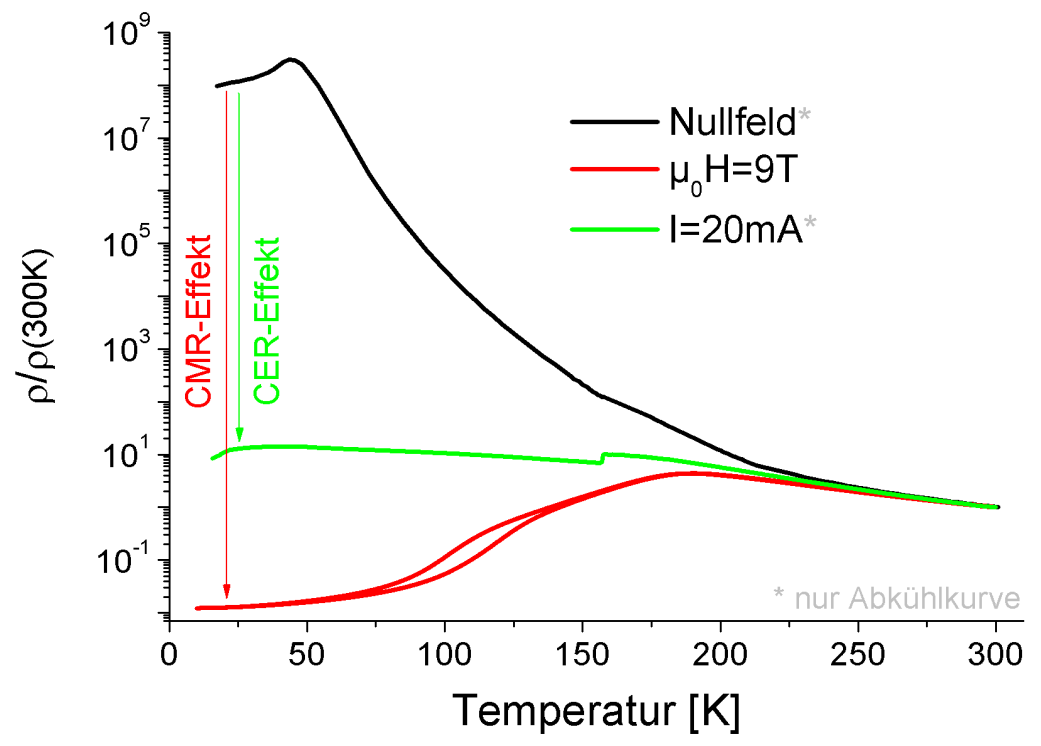

Abbildung 2.8: Normierter Widerstand aufgetragen über die Temperatur für eine Nullfeldmessung. Beispiele für das Auftreten des kolossalen Magnetowiderstands-Effektes (CMR-Effekt) und des kolossalen Elektrowiderstands-Effektes (CER-Effekt).

Neben dem kolossalen Magnetowiderstands-Effekt (CMR-Effekt) zeigt PCMO auch den kolossalen Elektrowiderstands-Effekt (CER-Effekt; siehe Abb. 2.8). Bei letzterem wird der Übergang in die ZP-geordnete Phase durch die entordnende Wirkung des elektrischen Stromes unterdrückt [42]. 


\section{Kapitel 3}

\section{Ergebnisse}

Die vorliegende Untersuchung zum PCMO befasst sich mit der Frage der Auswirkung von Defekten auf das elektrische Transportverhalten. Dazu wurden zum einen die Filmproben einer nachträglichen thermischen Behandlung unterzogen und in Abhängigkeit der Auslagerungszeit charakterisiert. Zudem sollten durch korrelierte Defekte, die über vizinale Substrate eingebracht wurden, gezielt Änderungen im elektrischen Verhalten induziert werden. Das Wechselspiel zwischen dem magnetischen bzw. dem elektrischen Feld und den Defekten ist der dritte Schwerpunkt in dieser Untersuchung.

Die elektrischen Transporteigenschaften sind in dieser Arbeit in Form des Widerstandes als Funktion der Temperatur, der Zeit sowie des angelegten magnetischen und elektrischen Feldes im Wechselspiel mit der Mikrostruktur (Defektdichte) untersucht worden. Durch die Betrachtungen der Transporteigenschaften in Abhängigkeit von der Zeit (Abschnitt 3.4.1) hat man einen Zugang zur Kinetik der Phasenübergänge. Die Transporteigenschaften im Zustand des CMR-Effektes werden in Abhängigkeit vom Magnetfeld (Abschnitt 3.2) sowie der Störung des CMR-Effektes durch Defekte (Abschnitt 3.3) untersucht. Auf die Auswirkungen der elektrischen Felder wird in Abschnitt 3.4.2 eingegangen. Durch das Wechselspiel von elektrischen und magnetischen Feldern (Abschnitt 3.4.2), insbesondere in der elektrisch leitfähigen Remanenz (Abschnitt 3.4.4) sollen Rückschlüsse auf metastabile Zwischenzustände in der Struktur ermöglicht werden.

Für die systematische Untersuchung der Transporteigenschaften sind die in Abb. 3.1 tabellarisch aufgetragenen Proben verwendeten worden. Neben der Depositionsmethode (PLD: gepulste Laserdeposition bzw. Sputtertechnik), der Her-

stellungstemperatur und der verwendeten Substratorientierung sind hier bereits vorab die Übergangstemperaturen aufgelistet. 


\begin{tabular}{|c|c|c|c|c|c|}
\hline & 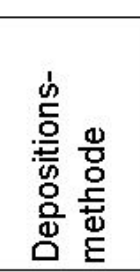 & 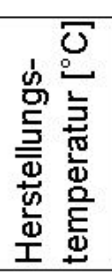 & 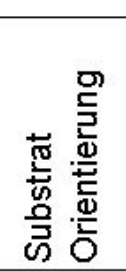 & 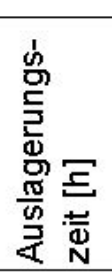 & 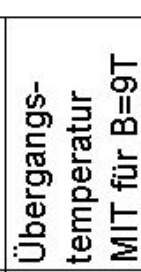 \\
\hline PLD-1_ap & PLD & 750 & planar & ohne & -- \\
\hline PLD-1_2h & & & & 2 & 69 \\
\hline PLD-1_5h & & & & 5 & 83 \\
\hline PLD-1_10h & & & & 10 & 102 \\
\hline PLD-1_20h & & & & 20 & 126 \\
\hline PLD-1_40h & & & & 40 & 170 \\
\hline PLD-1_80h & & & & 80 & 190 \\
\hline PLD-2_ap & PLD & 750 & planar & ohne & -- \\
\hline PLD-2_2h & & & & 2 & -- \\
\hline PLD-2_5h & & & & 5 & -- \\
\hline PLD-2_10h & & & & 10 & 45 \\
\hline PLD-2_20h & & & & 20 & 65 \\
\hline PLD-2_40h & & & & 40 & 81 \\
\hline PLD-2_80h & & & & 80 & 94 \\
\hline PLD-3_ap & PLD & 750 & planar & ohne & 150 \\
\hline PLD-4_ap & PLD & 750 & planar & ohne & 94 \\
\hline PLD-5_10h & PLD & 750 & planar & 10 & -- \\
\hline PLD-5_20h & & & & 20 & 90 \\
\hline Viz-1_20h & PLD & 750 & $9,5^{\circ}$ & $20^{*}$ & 163 \\
\hline Viz-2_2h & PLD & 750 & $9,5^{\circ}$ & 2 & 100 \\
\hline Viz-3_3h & PLD & 750 & $9,5^{\circ}$ & 3 & 93 \\
\hline Viz-4_ap & PLD & 750 & $3^{\circ}$ & ohne & n.a. \\
\hline Viz-5_ap & PLD & 750 & $6^{\circ}$ & ohne & n.a. \\
\hline Viz-6_20h & PLD & 750 & $6^{\circ}$ & 20 & 63 \\
\hline Spu-1_ap & Sputter & 650 & planar & ohne & -- \\
\hline Spu-1_20h & & & & 20 & --- \\
\hline Spu-2_ap & Sputter & 750 & planar & ohne & 75 \\
\hline
\end{tabular}

Abbildung 3.1: Übersicht der wichtigsten verwendeten Proben.

Die Auslagerungszeit findet sich auch im Probennamen wieder, da diese einen wichtigen Anhaltspunkt für die Defektdichte darstellt. 


\subsection{Strukturuntersuchungen}

\subsubsection{Oberflächenuntersuchungen}

Der erste Abschnitt befasst sich mit der Oberflächenmorphologie von wie hergestellten Proben sowie deren Änderungen nach thermischer Behandlung bei $900^{\circ} \mathrm{C}$ an Luft. Dazu ist zunächst zu berücksichtigen, dass selbst scheinbar identisch hergestellte Proben deutlich unterschiedliche Oberflächenmorphologien aufweisen können. Dies ist in Abbildung 3.2 anhand von AFM-Aufnahmen zweier Proben zu sehen. Für die Probe PLD-1 findet sich nach der Herstellung eine Oberfläche

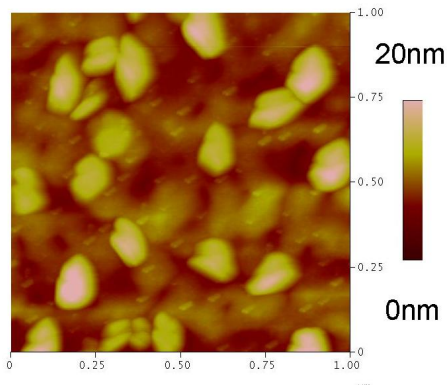

wie hergestellt (PLD-1)

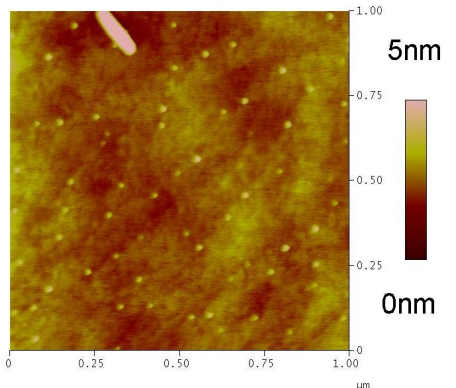

wie hergestellt (PLD-2)

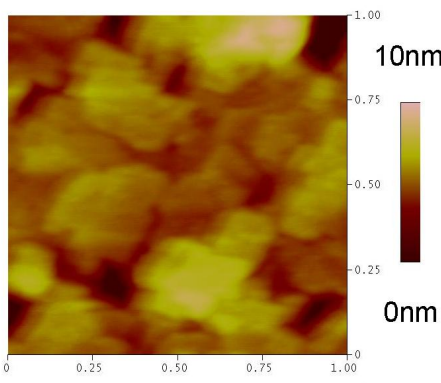

nach $5 \mathrm{~h}$ bei $900^{\circ} \mathrm{C}$

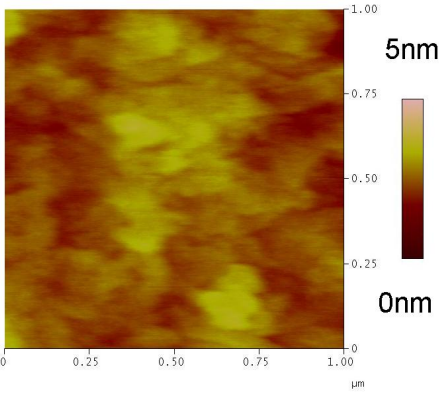

nach $5 \mathrm{~h}$ bei $900^{\circ} \mathrm{C}$

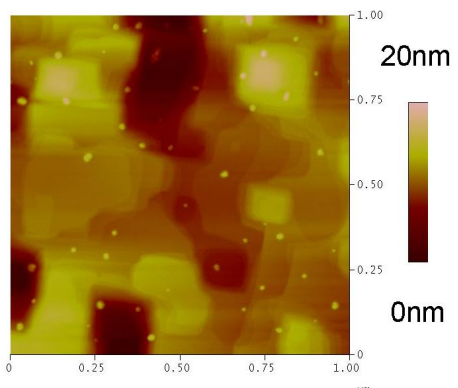

nach $40 \mathrm{~h}$ bei $900^{\circ} \mathrm{C}$

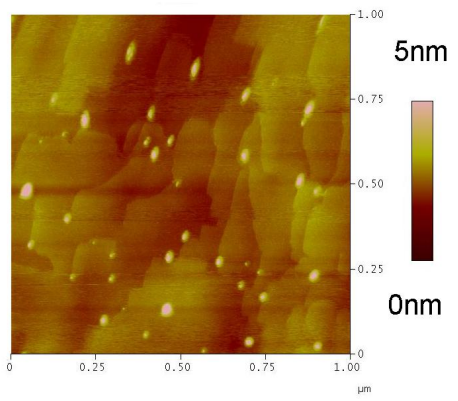

nach $40 \mathrm{~h}$ bei $900^{\circ} \mathrm{C}$

Abbildung 3.2: AFM-Messungen $1 x 1 \mu m^{2}$ der Proben PLD-1 und PLD-2 für wie hergestellt sowie zwei Auslagerungszeiten (nach 5h und nach 40h).

mit vielen Wachstumsinseln. Mit zunehmender Auslagerungszeit verschwinden die relativ kleinen, aber deutlich ausgeprägten rundlichen Strukturen. Es überwiegen jetzt klar definierte rechteckige Strukturen, auf denen terrassenförmige Wachstumslagen zu erkennen sind. Bei der Probe PLD-2 sind keine rundlichen Strukturen nach der Herstellung zu finden. Auch hier bilden sich für größere Auslagerungszeiten ebenfalls klar ausgeprägte sichtbare Lagen aus. Bemerkenswert ist bei dieser Probe das Fehlen der rechteckigen Wachstumsinseln, was für epitaktisch gewachsene Schichten mit Perowskit Struktur typisch ist. Dies spiegelt 
sich auch in der mittleren quadratischen Rauheit (RMS) wieder. Sie beträgt für die erste Probe 1,5-3 nm, für die zweite nur 0,2-0,5 nm. Die Änderungen der Rauheiten über die Auslagerungszeit sind zu vernachlässigen. Nach den Langzeitauslagerungen finden sich auf beiden Proben noch sehr kleine partikelartige Strukturen, wobei allerdings nicht klar ist, ob es sich hierbei um eine Oberflächenkontaminierung handelt. Die Dichte der bei der Filmherstellung mittels gepulster Laserablation typischerweise auftretenden Droplets wurde durch die Optimierung des Aufbaus auf nahe Null minimiert.

Die Oberflächenmorphologie auf vizinalen Substraten ändert sich mit dem Ver-
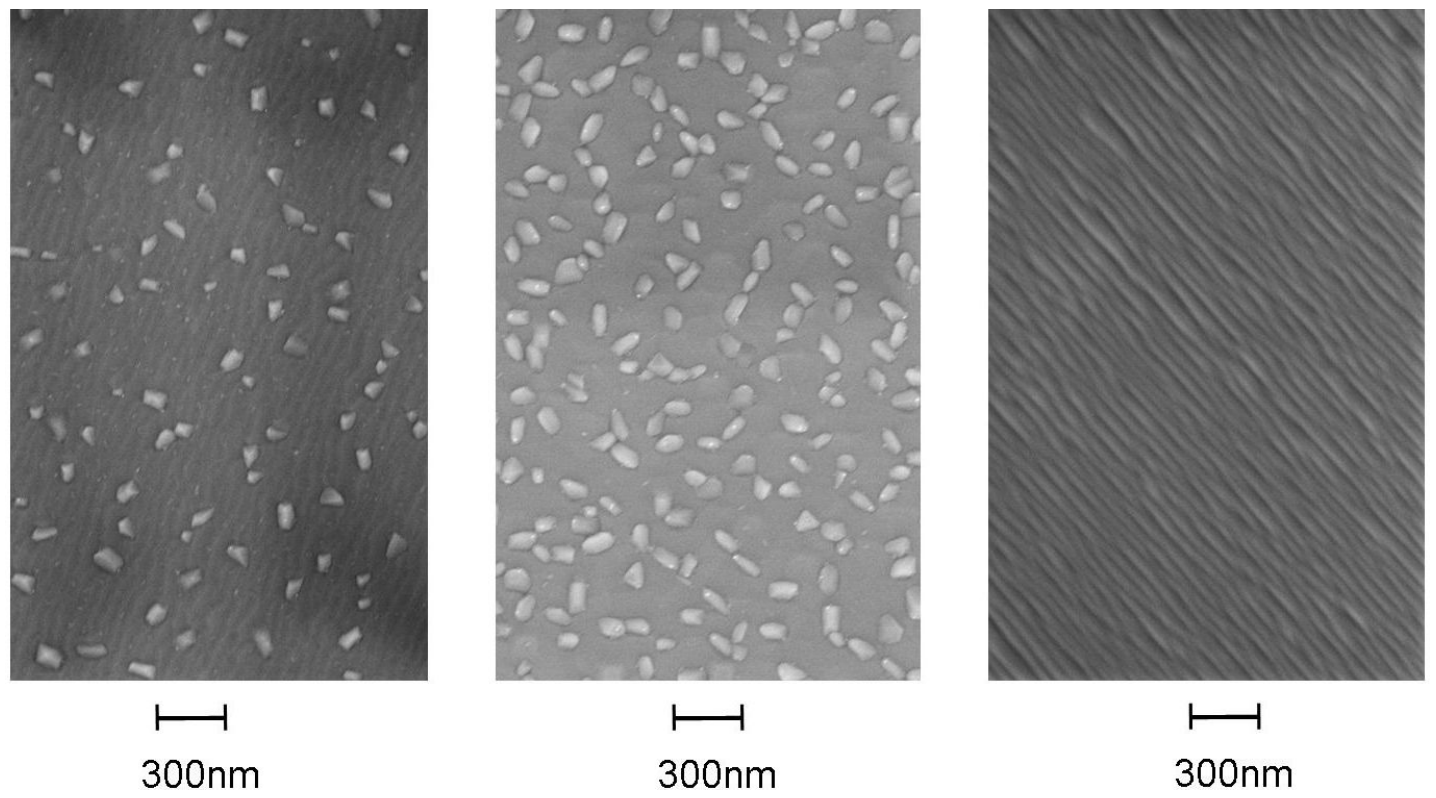

Abbildung 3.3: REM Aufnahmen der Oberflächenmorphologie für die Verkippwinkel $3^{\circ}, 6^{\circ}$ und $9,5^{\circ}$ (v.l.n.r.).

kippwinkel. Sind für die $3^{\circ}$ verkippte Probe noch viele Wachstumsinseln zu sehen, so verschwinden diese mit zunehmenden Verkippwinkel. Dafür bildet sich immer deutlicher sichtbar eine Stufen- bzw. Wellenstruktur aus, die durch die Substratstufen induziert werden. Es kommt auf vizinalen Substraten für größere Verkippwinkel zum Übergang vom Insel-Wachstum zum „step-flow"-Wachstum (siehe Abb. 3.3).

\subsubsection{Spannungsanalyse durch Röntgenbeugung}

Beim epitaktischen Wachstum dünner Filme werden durch die auftretenden mechanischen Spannungen Ebenenabstände eingestellt, die in der Regel von den 
Gitterparametern der Einkristalle abweichen. Für die Betrachtung der Ebenenabstände wurden die stärksten Reflexe (004) sowie (220) des Röntgendiffraktogrammes in einer $\Theta-2 \Theta$ Messung untersucht. Um die Vergleichbarkeit zu gewährleisten, wurden alle Messungen auf den STO-(002)-Reflex geeicht.

In dieser Geometrie spiegelt der (004) Reflex die c-Achsen-orientierten Zwillinge mit den Orientierungen PCMO[110][1-10]//STO[100][010] bzw. PCMO[110][110]//STO[100] [010] wieder. Diese beiden Orientierungen bzw. Zwillinge können bei diesen Messungen nicht weiter unterschieden werden. Auch allgemein wäre aus Röntgendiffraktogrammen auf Grund der geringen Unterschiede in der Länge der a- und b-Achse von etwa $0,05 \AA$ eine Unterscheidung äußerst schwierig. Aus dem (220) Reflex kann auf den [110]-Gitterparametern der vier Zwillinge mit der c-Achse in der Ebene geschlossen werden.

Wie sich die Spannungen in den Filmen während der Herstellung einstellen,

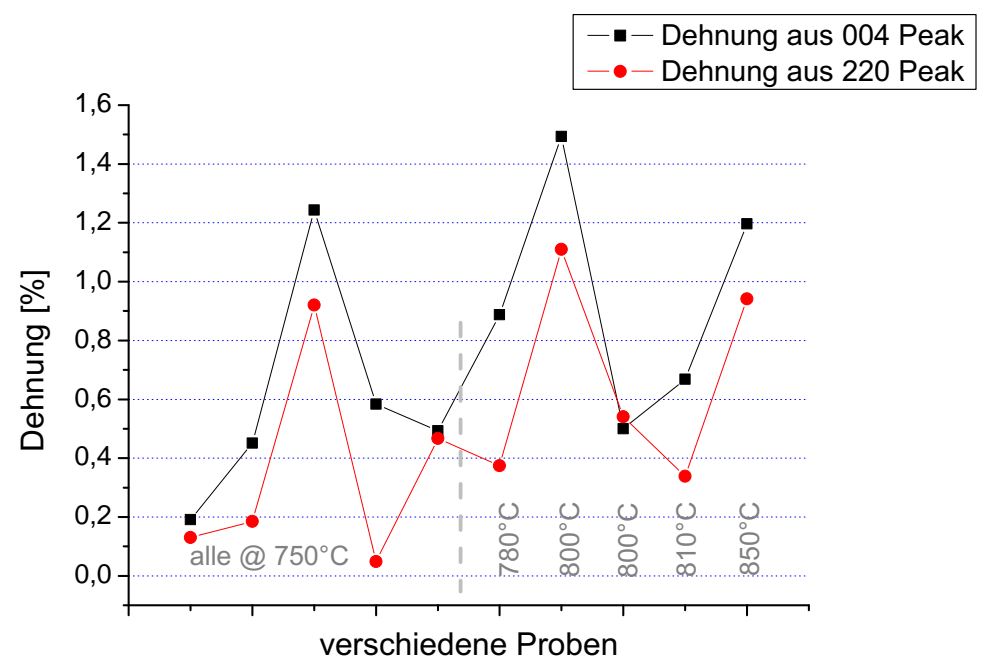

Abbildung 3.4: Aus den Reflexlagen der Röntgendiffraktogramme bestimmte Dehnungen für verschiedene Herstellungstemperatur.

hängt von vielen Faktoren ab. Diese können untereinander kompliziert gekoppelt sein. Zu berücksichtigen sind im Allgemeinen der Unterschied in den thermischen Ausdehnungen des Gitters zum Film, die Gitterfehlpassung zwischen Film und Substrat sowie herstellungsbedingte Defekte (z. B. durch hochenergetische Ionen), die selbst bei relativ hohen Depositionstemperaturen nur partiell ausheilen. Die Variation eines Herstellungsparameters kann so in Filmen zu unterschiedlichen Spannungswerten führen. In Abbildung 3.4 sind die Dehnungen $\epsilon_{H K L}=\frac{d_{H K L}^{F i l m}-d_{H K L}^{B u l k}}{d_{H K L}^{B u k}}$ für verschiedene Herstellungstemperaturen aufgetragen. Es 
ist keine Korrelation mit der Herstellungstemperatur zu erkennen. Selbst bei einer festen Temperatur $\left(750^{\circ} \mathrm{C}\right.$ in Abb. 3.4) sind die Dehnungen von Probe zu Probe verschieden. Dies zeigt, dass die Ebenenabstände in wie hergestelltem $\mathrm{Zu}-$ stand empfindlich von den Details der Deposition abhängen und nicht allein z. B. durch Fehlpassung zum Substrat bestimmt sind. In Abbildung 3.5 sind die
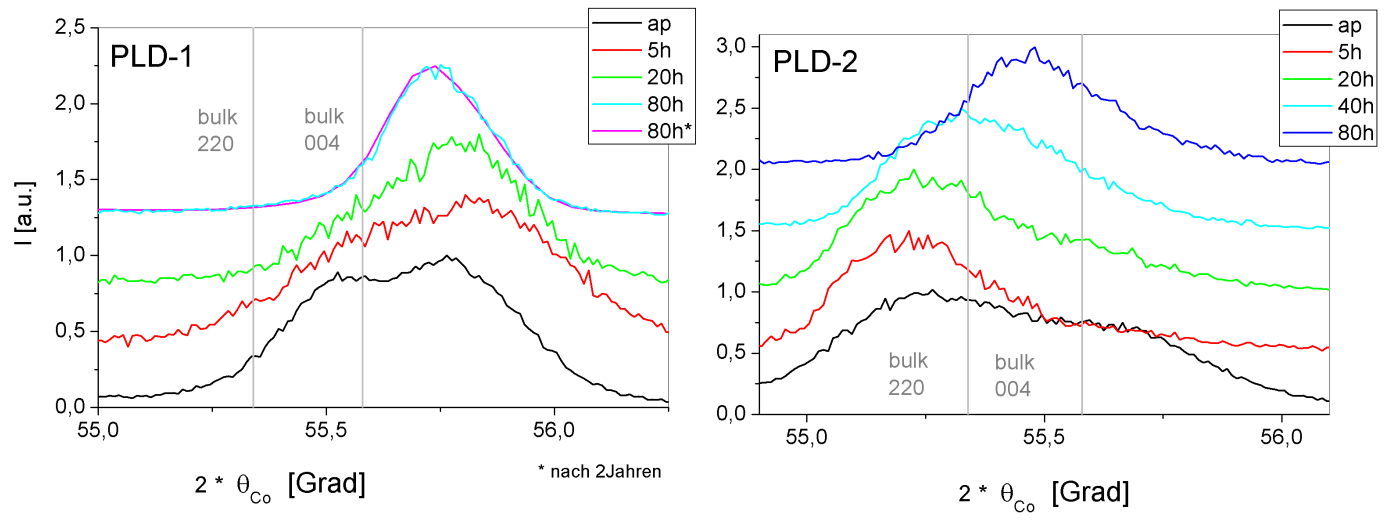

Abbildung 3.5: Röntgendiffraktogramme für die Proben PLD-1 und PLD-2 für verschiedene Auslagerungszeiten (Herstellungstemperatur $750^{\circ} \mathrm{C}$, Auslagerungstemperatur $\left.900^{\circ} \mathrm{C}\right)$.

Röntgendiffraktogramme für zwei identisch hergestellte Proben PLD-1 und PLD2 aufgetragen. In beiden sind die (004) sowie (220) Reflexlagen für unverspannte Einkristallproben mit eingezeichnet. Für die Messungen an den wie hergestellten Proben sind die Verzwillingungen gut zu erkennen, wobei die Probe PLD-1 für beide Zwillingsdomänen in der Ebene unter Dehnung steht und die Probe PLD2 unter Kompression. Auffällig bei Probe PLD-1 ist die Änderung des Anteils der Zwillinge zu Gunsten der c-Achsen orientierten Domänen mit zunehmender Auslagerungszeit. Dementgegen ist die Änderung der Reflexlage über der Auslagerungszeit nur sehr gering. Für die Probe PLD-2 gilt eher das Gegenteil. Zwar ändern sich ebenso die Anteile der Zwillinge, aber zu Gunsten des (110) orientierten Zwillings. Für sehr lange Auslagerungszeiten kommt es zusätzlich zu einer Änderung der Reflexlage in Richtung des Einkristall-Wertes. Dabei spielen Alterungseffekte der Proben keine Rolle. In Abbildung 3.5 ist für die Probe PLD-1 $1_{80 h}$ eine Wiederholung der Messung nach 2 Jahren (ohne weitere Auslagerung) zu sehen. Die Messungen zeigen genau den gleichen Verlauf. 


\subsubsection{TEM Untersuchungen}

Wie bereits aus den Röntgendiffraktogrammen ersichtlich wurde, ist die Struktur geprägt von den Zwillingen. Dabei können diese sehr unterschiedliche Größen haben. In wie hergestellten Proben haben TEM-Untersuchungen ${ }^{1}$ ergeben, dass es

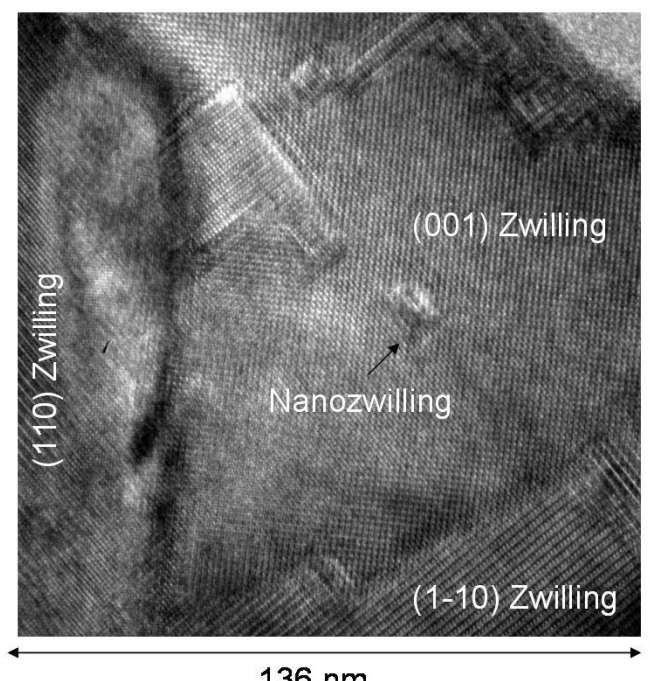

$136 \mathrm{~nm}$

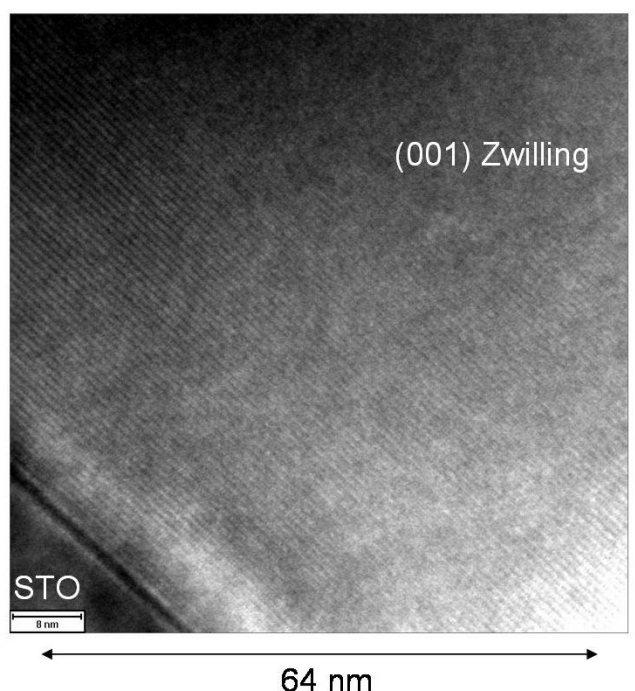

$64 \mathrm{~nm}$

Abbildung 3.6: TEM-Aufnahmen links: „plane view“ einer wie hergestellten Probe mit Zwillingen und Nanozwillingen, rechts: „cross-section“ Aufnahme der Probe PLD- $1_{80 h}$ ohne Zwillingsbildung.

Zwillinge mit Größen bis in den nanometer Bereich gibt. Ein Beispielbild ist in Abbildung 3.6 links zu sehen. Solche Nanozwillinge sind aber nur typisch für wie hergestellte Proben. Sie sind nach einer Auslagerung nicht mehr zu beobachten (Abb. 3.6 rechts). Durch sehr lange Auslagerungszeiten können Zwillinge mit einer Größe einer TEM-Lamelle (ca. $10 \mu \mathrm{m}$ ) entstehen. Durch das Wachstum auf vizinalen Substraten können Fehler im Kippsystem entstehen, die Anti-Phasen-Grenzen (APB) ausbilden. Ausgangspunkt dieser APB ist die gestufte Oberfläche des vizinalen Substrates. Ein Beispiel hierfür zeigt Abb. 2.5, allerdings wurde weder eine regelmäßige Bildung von $\mathrm{APB}$ an den Stufen, noch ein typischer Abstand der APB beobachtet. Solche planaren Defekte spielen eine wesentliche Rolle bei der Ordnungseinstellung, da sie Nukleationszentren für die geordnete Phase darstellen. Dies ist für einen ausgedehnten Defekt, der gleichzeitig als Markierung diente in Abb. 3.7 gezeigt. Bei Raumtemperatur liegt nur ein kleiner Volumenanteil der geordneten Phase vor, der beim Abkühlen deutlich zunimmt. Die Abbildung 3.7

\footnotetext{
${ }^{1}$ TEM Aufnahmen von Jonas Norpoth sowie Christian Jooß, teilweise in Zusammenarbeit mit dem BNL (USA)
} 


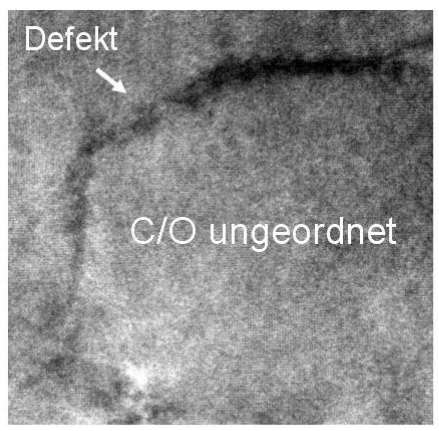

RT

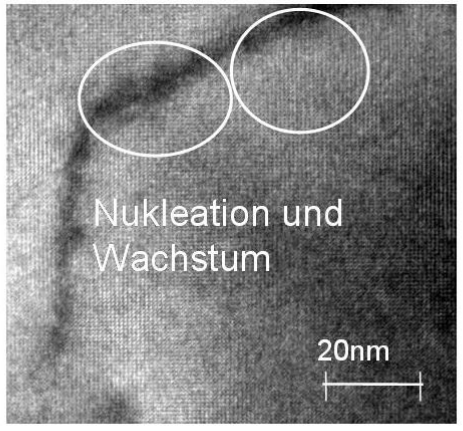

$162 \mathrm{~K}$

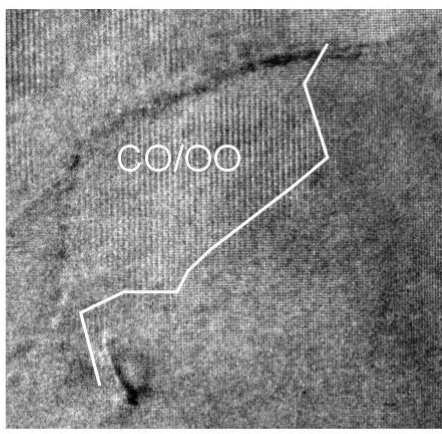

$85 \mathrm{~K}$

Abbildung 3.7: TEM-Aufnahmen des Wachstums der OO/CO Phase an einem ausgedehnten Defekt.

zeigt die Keimbildung und das Wachstum der geordneten Phase bei einer solchen Abkühlung, wobei die Keimbildung an einem planaren Defekt stattfindet.

Linienhafte Defekte, die ebenfalls als Keimbildungszentren wirken könnten, traten in Form von Partialversetzungen nur in Verbindung mit Anti-Phasen-Grenzen auf.

\subsubsection{SQUID Magnetisierungsmessungen}

In diesem Abschnitt wird auf die Auswirkung von Defekten auf die magnetischen Eigenschaften eingegangen. Defekte können sowohl die Ausbildung der antiferromagnetisch-geordneten Phase als auch der ferromagnetisch-metallartigen Phase stark beeinflussen, bzw. sogar unterdrücken.

Im Folgenden werden die Ergebnisse zu den magnetischen Eigenschaften am Beispiel dreier Proben vorgestellt. Die Auswahl erfolgte dabei über die Leitfähigkeit: Probe 1 ist aus der Auslagerungsserie (Probe PLD-1). Sie zeigt, wie in späteren Abschnitten dargestellt, sehr gute elektrische Eigenschaften in der Form eines ausgeprägtem CMR $\left(T_{M I T}(9 T) \approx 190 K\right)$. Die Probe P3 zeigte selbst nach einer nachträglichen Auslagerung keinen Metall-Isolator-Übergang. Die Probe PLD-2 liegt vom elektrischen Transport aus gesehen mit einer Übergangstemperatur von 93K zwischen diesen beiden Extremen. Die im Vordergrund stehende Fragestellung ist, in wie fern sich Unterschiede in den elektrischen Eigenschaften im Magnetismus widerspiegeln. Für PCMO $\mathrm{x}=0,32$ erwartet man für hohe Temperaturen bis zur Néel-Temperatur $\left(T_{\text {Néel }} \approx 140 \mathrm{~K}\right.$, [83]) paramagnetisches Verhalten, darunter antiferromagnetisches Verhalten. Des Weiteren wird ein Übergang zum verkanteten Antiferromagnetismus bei etwa $100 \mathrm{~K}$ diskutiert. In einem hinreichend starken Magnetfeld kann unterhalb der Ordnungstemperatur (2.1) in PCMO eine ferromagnetische Ordnung induziert werden. Betrachtet man die Magnetisierungskur- 


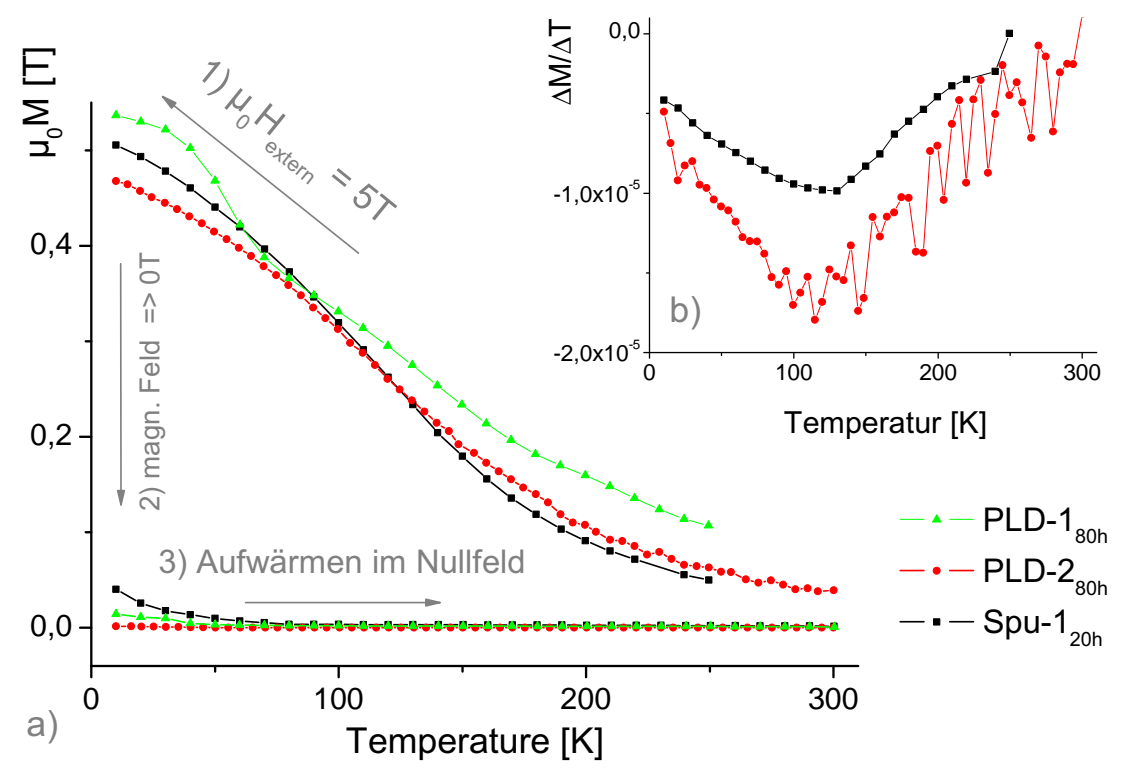

Abbildung 3.8: a) Magnetisierung in Abhängigkeit von der Temperatur; Abkühlkurven in einem Magnetfeld von 5T, Aufwärmkurve anschließend im Nullfeld; der diamagnetische Beitrag des STO-Substrates wurde subtrahiert; b) zugehörige Ableitung der Magnetisierung nach der Temperatur über die Temperatur.

ven über die Temperatur im Magnetfeld (Abb. 3.8 a) für die Probe Spu- $1_{20 h}$ sowie die Probe PLD- $2_{80 h}$, so sind keine Anzeichen für einen magnetischen Übergang zu sehen. Das Maximum der negativen Steigung $-\frac{\delta M}{\delta T}$ liegt für beide Proben etwa bei 115K (Abb. 3.8 b), was weder mit den magnetischen Übergangstemperatur noch mit der elektrischen Übergangstemperatur korreliert. Die Probe PLD- $1_{80 h}$ hat ein Maximum in der Steigung bei etwa 215K, was mit der Übergangstemperatur für die Ladungs- und Orbitalordnung in Verbindung gebracht werden kann. Ein weiteres Maximum befindet sich bei 140K, welches mit der Néel-Temperatur zusammen fällt. Das Minimum bei etwa 100K könnte vom Übergang in den verkanteten Antiferromagnetismus verursacht werden (Abb. 4.5). Nach dem Abschalten des Magnetfeldes bei 10K verbleibt eine magnetische Remanenz (Probe Spu- $1_{20 h}$ 40mT; Probe PLD- $2_{80 h} 1,5 \mathrm{mT}$; Probe PLD- $1_{80 h} 14 \mathrm{mT}$ ), wobei diese Remanenz für alle Proben bei ca. 80K verschwindet. Ein möglicher Zusammenhang zur elektrischen Remanenz wird in der Diskussion näher beleuchtet. Betrachtet man das Verhalten der Magnetisierung über das Magnetfeld für feste Temperaturen (Abb. 3.9), so zeigen alle untersuchten Proben bei Temperaturen oberhalb der NéelTemperatur das erwartete paramagnetische Verhalten (z. B. 200K-Messung in 


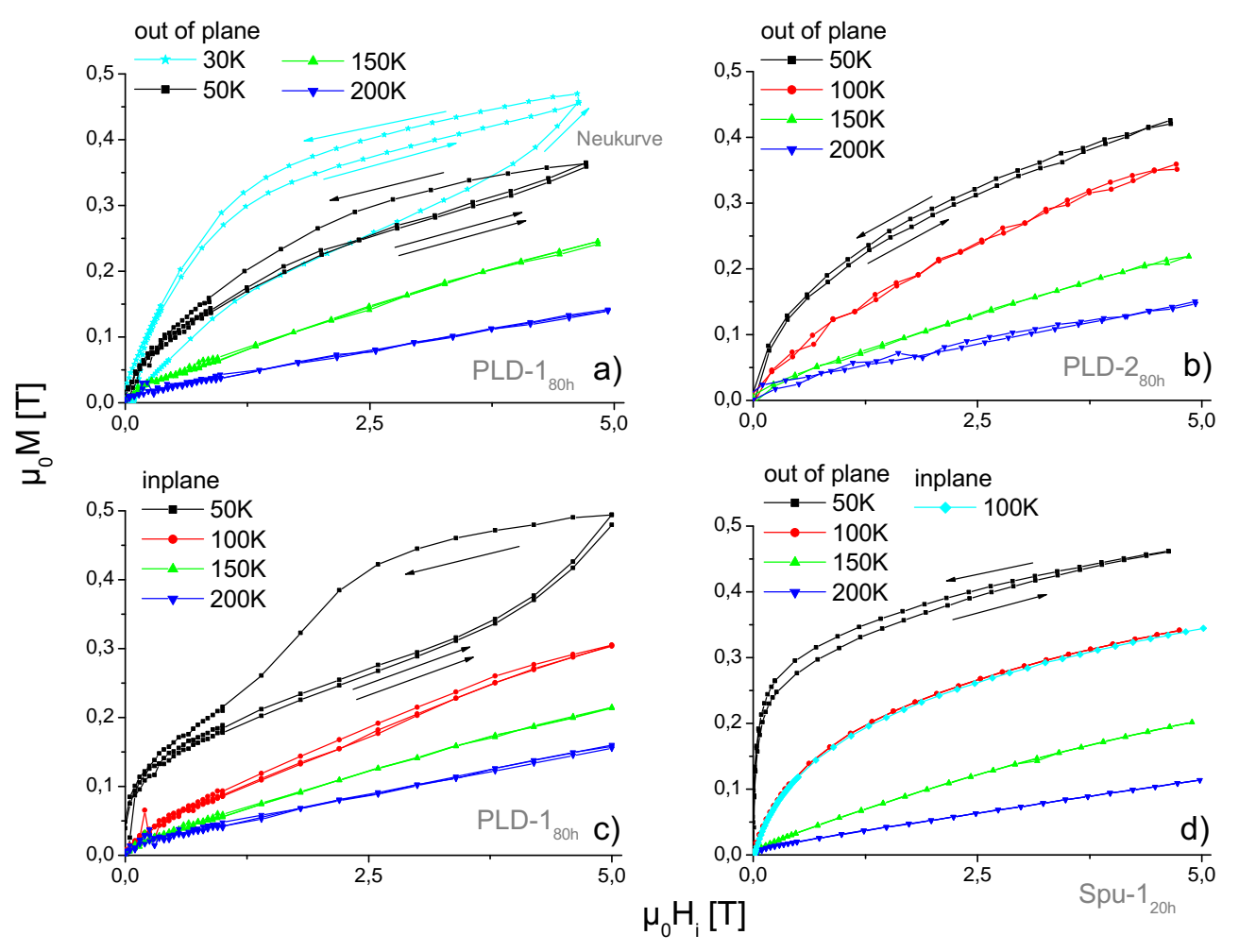

Abbildung 3.9: Magnetisierung in Abhängigkeit vom inneren Magnetfeld $\mu_{0} H_{i}$ für verschiedene Temperaturen; In allen Messungen Abzug des Substratanteils.

Abb. 3.9). Unterhalb der Néel-Temperatur zeigen sowohl die Probe Spu- $1_{20 h}$, wie auch die Probe PLD- $2_{80 h}$ antiferromagnetisches Verhalten, wobei die Probe Spu$1_{20 h}$ ein großes Magnetisches Moment sowie eine höhere effektive Suszeptibilität $\frac{d M}{d B}$ für kleine Felder zeigt. In den Messungen für $50 \mathrm{~K}$ zeigen beide Proben eine kleine Hysterese, die Probe PLD- $1_{80 h}$ eine ausgeprägte Hysterese. Wie man in der Messung c erkennen kann (das Magnetfeld ist hier parallel zum Substrat gerichtet, ,in-plane“), ist die Hysterese mit einem Übergang zum Ferromagnetismus bei ca. 3,5T verbunden. Das größere magnetische Moment wird bei Felderniedrigung auch bis ca. 3T beibehalten, bevor es dann wieder auf den Wert des antiferromagnetischen Astes abfällt. Für die gleiche Messung mit dem Magnetfeld senkrecht zur Filmebene (Abb. 3.9 a, 50K) ist der Übergang in den Ferromagnetismus viel weniger ausgeprägt, aber immer noch stärker als für die anderen beiden Proben. Die Kristallanisotropie im Magnetismus spiegelt sich auch bei anderen Temperaturen für die Probe PLD- $1_{80 h}$ in Form von unterschiedlichen Anstiegen der 
Magnetisierung wieder. Im Gegensatz dazu ist in der Probe Spu- $1_{20 h}$ keine Anisotropie im Magnetismus zu finden, wie anhand der 100K Messung (Abb. 3.9 d) gezeigt worden ist. Die Kurven liegen faktisch übereinander. Wie später diskutiert wird, spielen die Zwillinge und deren Anteile eine große Rolle für diese Effekte sowie die Unterschiede im magnetischem Verhalten. Ein weiterer interessanter Unterschied ist im Vergleich der 30K und 50K Messungen (Abb. 3.9 a) zu finden. Während bei $50 \mathrm{~K}$ die Neukurve und der entsprechende Zweig der Hystereseschleife fast übereinander liegen, so ist bei $30 \mathrm{~K}$ eine Neukurve zu finden, die unterhalb der eigentlichen Hystereseschleife liegt. Während bei 50K der Übergang vom antiferromagnetischen Zustand in einen metastabilen ferromagnetischen Zustand induziert wird, kommt es bei 30K zur Ausbildung einer stabilen ferromagnetischen Ordnung. Die Gründe hierfür sollen im Zusammenhang mit den elektrischen Messungen sowie Strukturuntersuchungen im Folgenden Kapitel 4 diskutiert werden. Das mit $5 \mathrm{~T}$ erreichte Moment beträgt $3,1 \mu_{B} / \mathrm{Mn}$ Atom, wobei die $5 \mathrm{~T}$ allerdings noch nicht für eine vollständige Sättigung ausreichten. Das maximal mögliche Moment liegt bei 3,7 $\mu_{B} / \mathrm{Mn}$ Atom [41].

\subsubsection{Interaktion an der Grenzfläche zwischen Substrat und Film}

Durch die thermische Nachbehandlung bei $900^{\circ} \mathrm{C}$ ist für sehr lange Auslagerungszeiten eine Interdiffusion an der Grenzfläche zwischen Film und Substrat nicht mehr auszuschließen. Um dies zu untersuchen, wurde für verschieden lang ausgelagerte Proben die Zusammensetzung der Grenzfläche mittels SekundärionenMassenspektrometrie (SIMS) bestimmt. In Abb. 3.10 sind die Konzentrationsverläufe für die verschiedenen Elemente aus Film und Substrat dargestellt. Das Signal (detektierte Teilchen pro Zeiteinheit) ist auf die Werte im reinen Film bzw. Substrat normiert.Vergleicht man die verschieden ausgelagerten Proben, so wird deutlich (insbesondere für die 80h Auslagerung), dass anscheinend Strontium in den PCMO Film diffundiert ist, während das STO-Substrat Praseodym sowie Kalzium enthält. Eine solche Substitution scheint plausibel, da $\mathrm{CaTiO}_{3}$ und $\operatorname{Pr}_{1-x}\left(\mathrm{Ca}_{1-y} \mathrm{Sr}_{y}\right)_{x} \mathrm{MnO}_{3}$ [84] wohlbekannte Phasen sind. Ausgehend von einer Schichtdicke der Filme von 330nm und der Zeit bis zum Erreichen der Grenzfläche $^{2}$, ergibt sich bei der Sputtererrosion eine Abtragsrate von ca. $1 \mathrm{~nm}$ je Minute. Die Verschmierung der Grenzfläche in den SIMS-Spektren beträgt somit ca. 25nm, was der Rauheit (Abstand zwischen dem höchsten und dem niedrigsten Punkt des Oberflächenprofiles) der Oberfläche für die untersuchten Filme entspricht. Die Interdiffusionszone für das Strontium entspricht für den 80h ausgelagerten Film etwa $15 \mathrm{~nm}$ in den Film, sowie für Kalzium in das Substrat von etwa. 10nm. Bereits im wie hergestellten Film scheint eine Interdiffusionszone

\footnotetext{
${ }^{2}$ Ausgehend von der Annahme, dass die Mangan-Diffusion vernachlässigt werden kann, wurde die Grenzfläche an die Stelle gesetzt, bei der sich das Mn-Signal halbiert hat
} 


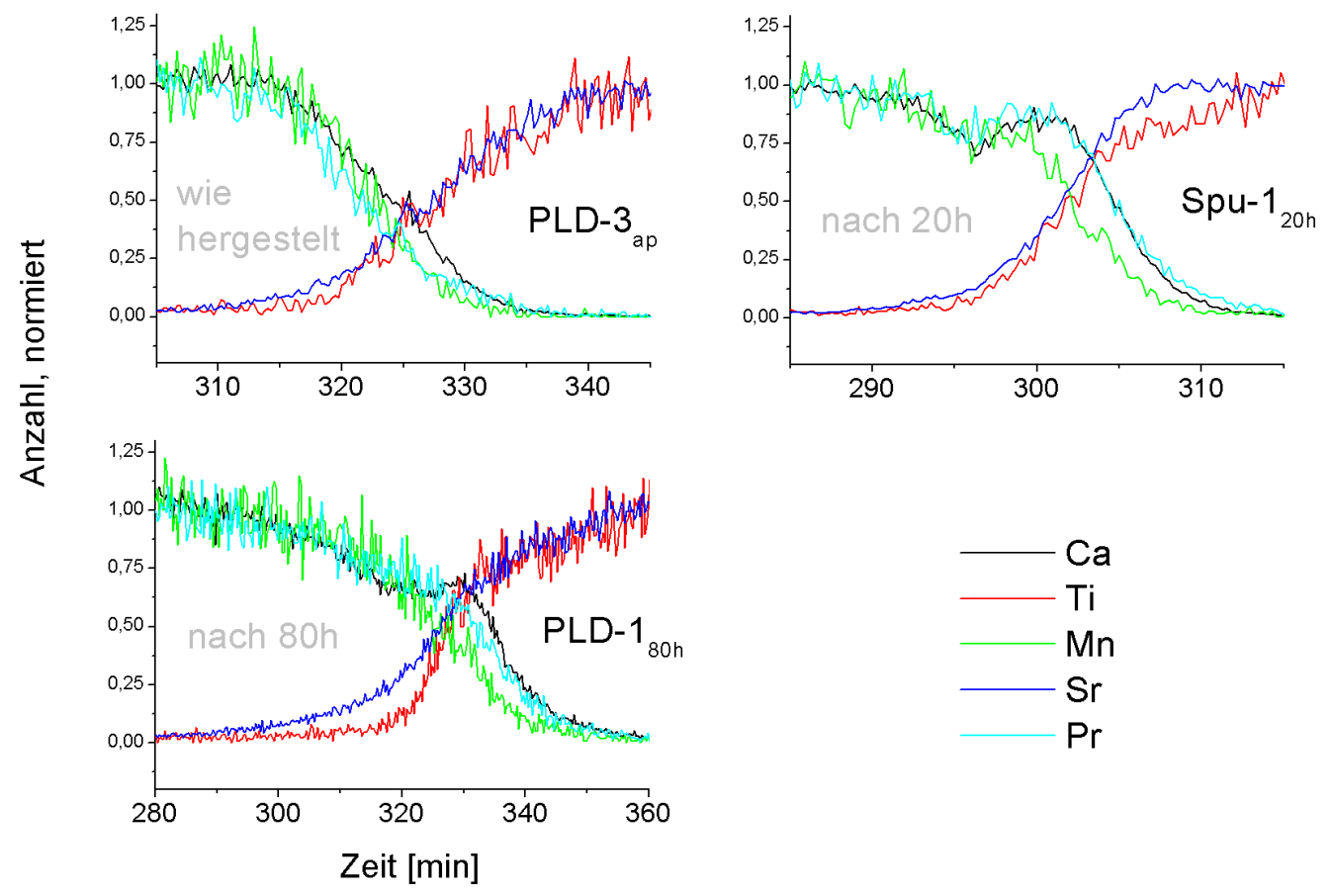

Abbildung 3.10: Sekundärionen-Massenspektrometrie Messungen für verschieden lang ausgelagerte Proben; Herstellungstemperatur PLD Proben $750^{\circ} \mathrm{C}$, gesputterte Probe $650^{\circ} \mathrm{C}$.

von maximal ca. 2nm vom Kalzium in das Substrat zu existieren, siehe kleine Differenz im Spektrum der wie hergestellten Probe, Abb. 3.10. Bei der Probe Spu- $1_{20 h}$ ist ebenso eine Interdiffusionszone von Kalzium in das Substrat von 2nm zu erkennen, wobei auch hier vielleicht zusätzlich das Praseodym in das Substrat diffundiert sein könnte.

\subsection{Der Kolossale Magnet-Widerstands-Effekt}

Die hervorstechende Eigenschaft der Manganate ist die Möglichkeit den elektrischen Widerstand durch externe Felder um viele Größenordnungen zu ändern. In Abbildung 3.11 ist exemplarisch der spezifische Widerstand in einem Magnetfeld von 9T über der Temperatur aufgetragen. Während sich der Widerstand ohne äußere Einflüsse über den gesamten Temperaturbereich isolierend verhalten würde, so kommt es in einem hinreichend starken Magnetfeld zu einem Isolator-Metall 


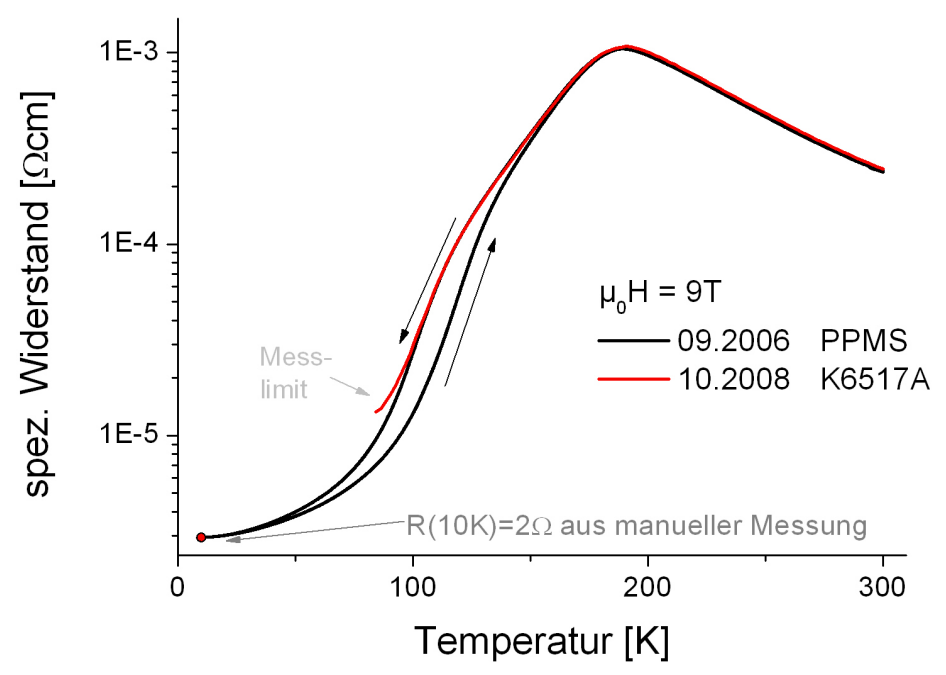

Abbildung 3.11: Transportmessung spezifischer Widerstand über die Temperatur in einem Magnetfeld von 9T und die Wiederholung der Messung nach 2 Jahren; Probe PLD- $1_{80 h}$.

Übergang ${ }^{3}$. Die Temperatur beim maximalen Widerstand wird im Folgenden mit $T_{c}$ bezeichnet. Dabei tritt zwischen der Abkühl- und der Aufwärmkurve eine Hysterese im Widerstand auf. Dieser Bereich, der fast immer mit der starken Änderung des Widerstandes verbunden ist, wird im Folgenden als Übergangsbereich bezeichnet.

Bei einer so drastischen Änderung ist eine gute Langzeitstabilität und Reproduzierbarkeit keine Selbstverständlichkeit. In Abbildung 3.11 ist eine Wiederholung einer Messung zu sehen, welche nach zwei Jahren an der selben Probe durchgeführt wurde. Die elektrischen Transporteigenschaften sind nahezu identisch ${ }^{4}$. Einige Proben zeigten im wie hergestellten Zustand keinen Metall-Isolator Übergang. Dieser tritt erst nach einer nachträglichen Temperaturbehandlung ${ }^{5}$ auf. Die Veränderung hängt dabei deutlich von der Auslagerungszeit ab. In Abbildung 3.12 sind für zwei Proben (PLD-1 und PLD-2) die Transporteigenschaften für verschiedene Auslagerungszeiten aufgetragen. Die Übergangstemperatur bzw. der Übergangsbereich verschiebt sich mit zunehmender Auslagerungszeit zu höheren Temperaturen (Abb. 3.12 rechts unten). Dabei sinkt der Raumtemperaturwiderstand in der ersten Auslagerungsstufe stark, im weiteren nur vergleichsweise wenig ab. Der im Magnetfeld bei 9T im CMR erreichte Tieftemperaturwider-

\footnotetext{
${ }^{3}$ auch kurz Metall-Isolator Übergang, MIT

${ }^{4}$ In beiden Fällen Unterschied nur im Messequipment

${ }^{5}$ Auslagerung bei $900^{\circ} \mathrm{C}$ an Luft
} 

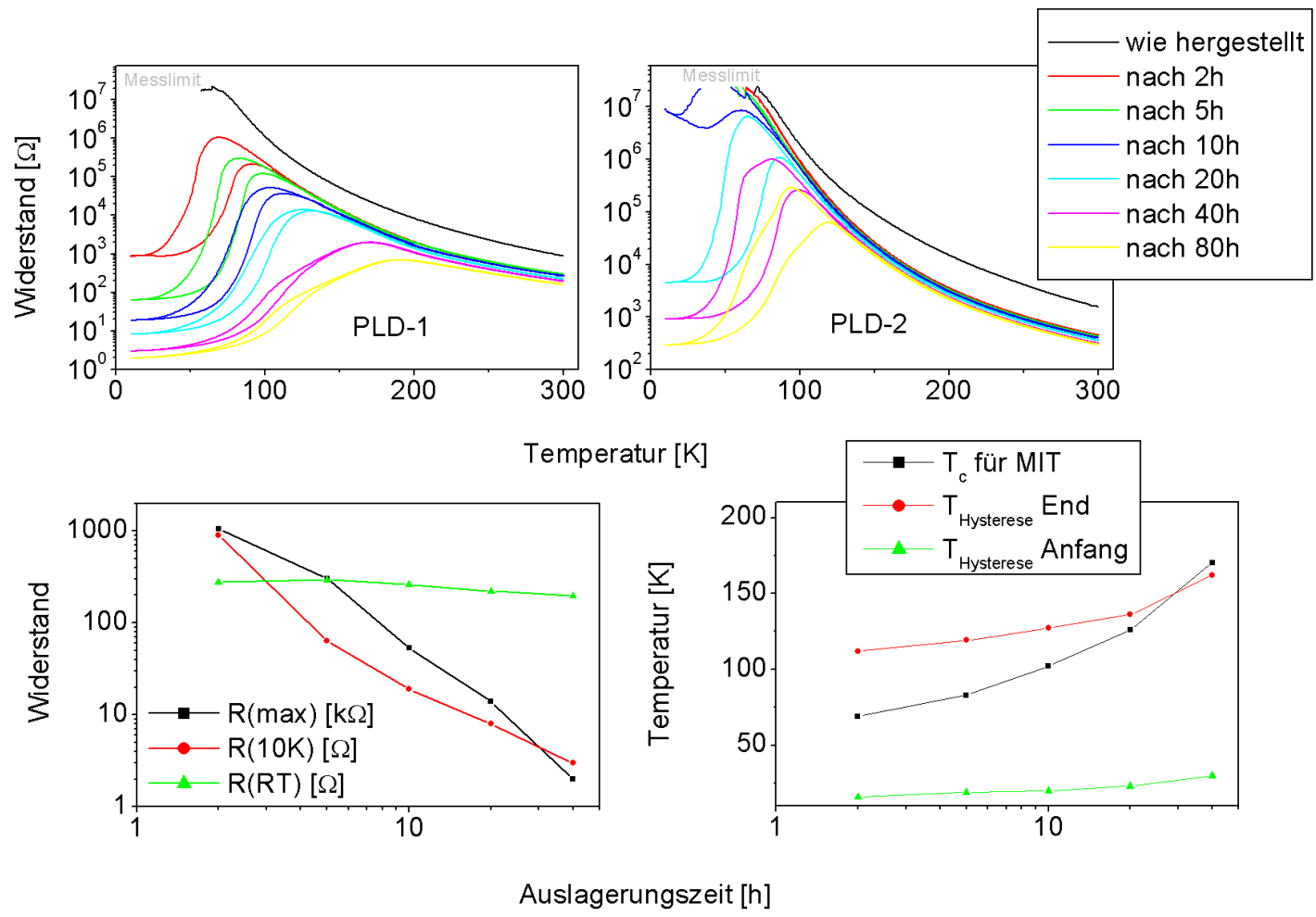

Abbildung 3.12: oben: Widerstand über die Temperatur für zwei Proben PLD-1 und PLD-2 in Abhängigkeit der Auslagerungszeit im Magnetfeld von 9T, unten: charakteristische Widerstände und Temperaturen für die Probe PLD-1.

stand (bei 10K) sowie der maximale Widerstand sind in Abbildung 3.12 links unten aufgetragen. Die signifikanten Einflüsse des Magnetfeldes und der Auslagerungszeit beschränken sich nicht alleine auf den Tieftemperaturbereich, sondern sind auch im Hochtemperaturbereich merklich. Diese werden besonders deutlich, wenn man anstelle des Widerstandes dessen logarithmische Ableitung betrachtet. Dazu geht man zunächst davon aus, dass der temperaturabhängige Widerstand ohne externes Feld sich gut im Rahmen des Modells der thermisch aktivierten Polaronenbewegleichkeit (TAP, siehe 2.3) beschreiben lässt. Wendet man dieses Modell nun formal auch auf den Hochtemperaturwiderstand im Magnetfeld an, so erhält man eine im Allgemeinen feld- und temperaturabhängige effektive Aktivierungsenergie $E_{a}$.

$$
E_{a}=\frac{d \ln \frac{\rho}{T}}{d \frac{1}{k_{B} T}}
$$

In Abbildung 3.13 links oben ist diese Größe für die Probe PLD-1 in einem Ma- 


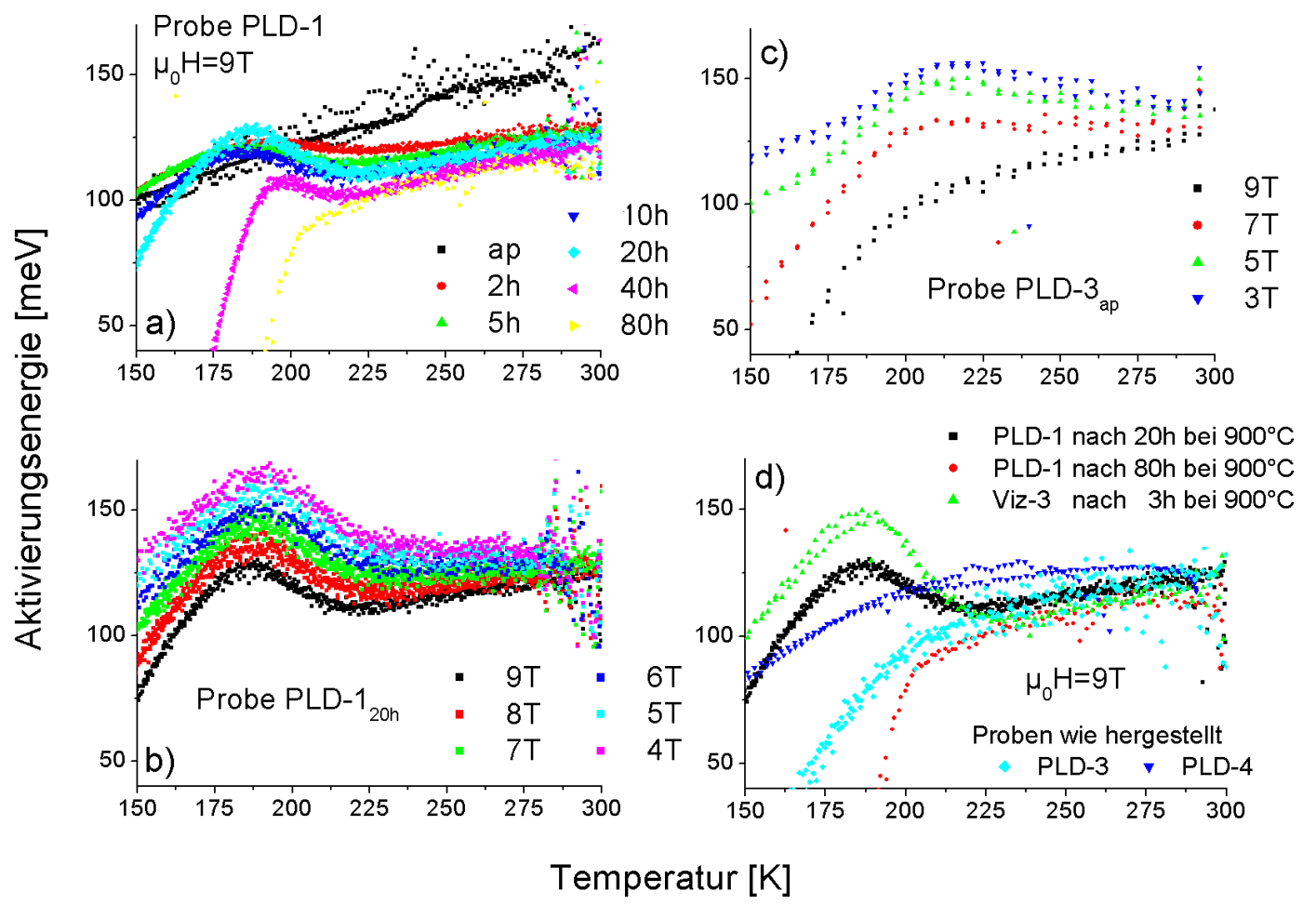

Abbildung 3.13: Probe PLD-1 der Auslagerungsserie: a) Vergleich der Aktivierungsenergie bezogen auf Auslagerungszeit in einem Magnetfeld von 9T; b) für eine feste Auslagerungszeit von 20h, Aktivierungsenergie für verschiedene Magnetfelder; c) wie b) jedoch Probe PLD-3 $3_{a p}$ direkt nach der Herstellung; d) Vergleich Proben (alle mit CMR-Effekt) mit und ohne nachträgliche Auslagerung.

gnetfeld von 9T dargestellt. Ohne Auslagerung liegt innerhalb des Gültigkeitsbereiches des TAP-Modells $\left(T \leq \frac{\Theta_{D}}{2} \approx 160 K\right)$ eine nahezu lineare Temperaturabhängigkeit vor. Nach einer Auslagerungszeit von 2h ist ein lokales Maximum der Aktivierungsenergie bei ca. 225K zu sehen. Dieser Anstieg der Aktivierungsenergie wird mit zunehmender Auslagerungszeit ausgeprägter. Wie die Teilabbildung b zeigt, wird das lokale Maximum mit zunehmendem Magnetfeld kleiner, und zwar um so stärker, je länger die Probe ausgelagert wird. Aus diesen gegenläufigen Tendenzen erklärt sich zum Beispiel, warum in hohen Feldern und nach langen Auslagerungen (vgl. a bzw. d) das lokale Maximum verschwindet. Obgleich es zwischen verschiedenen Proben im Detail Unterschiede gibt, so gibt es einige gemeinsame Tendenzen, wie auch das Teilbild c für eine andere Probe zeigt. Diese wies schon nach der Herstellung einen allerdings wenig ausgeprägten CMR auf. Die allgemeinen Regeln lassen sich in etwa wie folgt zusammenfassen. 
- Proben, die nach der Herstellung keinen oder nur einen schwach ausgeprägten CMR zeigen, lassen sich im Nullfeld zumindest ansatzweise im TAPModell beschreiben, dass heißt die scheinbare Aktivierungsenergie ist nur schwach temperaturabhängig.

- Proben, die zum Beispiel infolge einer Auslagerung einen ausgeprägten CMR aufweisen, zeigen in einem Temperaturbereich von ca. 180-230K ein Maximum in der Aktivierungsenergie, das umso stärker ausgeprägt ist, je länger die Probe ausgelagert wird. Dieser Temperaturbereich ist insofern bemerkenswert, da er zwischen den charakteristischen Ordnungstemperaturen $\left(T_{\text {Neel }} \approx 160 K, T_{C O} \approx 260 K\right)$ liegt.

- Das zusätzliche Anlegen eines Magnetfeldes führt zu einer linearen Abnahme der Aktivierungsenergie, sowohl bzgl. der abnehmenden Temperatur als auch mit zunehmenden Magnetfeld. Weiterhin unterdrückt das Magnetfeld das Maximum in der Aktivierungsenergie.

\subsection{Filme auf vizinalen Substraten}

Die im vorigen Kapitel vorgestellten Ergebnisse bezogen sich auf Proben, die auf planaren Substraten hergestellt wurden, bei denen man also davon ausgehen kann, dass die durch die Deposition bzw. durch die filmwachstumbedingten Defekte keine Vorzugsrichtung auf makroskopischer Längenskala aufweisen. Die Einschränkung auf makroskopischer Skala ist dabei für epitaktische Filme notwendig, da dabei generell kristallographische Orientierungen berücksichtigt werden müssen. Man kann aber davon ausgehen, dass zum Beispiel in zwei ansonsten beliebigen senkrechten Richtungen die Anzahl der Zwillingsgrenzen in der Filmebene gleich sind. Dies ändert sich, wenn man vizinale Substrate betrachtet. Durch die periodische Anordnung von Defekten können (siehe Kapitel 2.2.3), wie das Beispiel der Antiphasengrenzen zeigt, mesoskopische bis makroskopische Domänen mit anisotropen Transporteigenschaften entstehen. Wie die Abbildung 3.14 zeigt, ist eine solche Richtungsanisotropie im Widerstand in PCMO-Filmen auf $9,5^{\circ}$ verkippten Substraten zu beobachten. Eine deutliche Anisotrope im Trans-

portverhalten $\left(\frac{\rho_{\text {parallel }}}{\rho_{\text {senkrecht }}}\right)$ ist dabei allerdings nur im Übergangsbereich zu finden. Für hohe Temperaturen sind die Widerstände fast identisch.

Beschränkt man sich zunächst auf den Temperaturbereich oberhalb der Übergangstemperatur, so wird allerdings auch deutlich, dass mit abnehmender Temperatur eine kleine Anisotropie auftritt, der Widerstand parallel zur Richtung der Substratstufen ist kleiner als senkrecht zu ihnen. Dies impliziert im Sinne des vorigen Kapitels Unterschiede in den Aktivierungsenergien. Die Abbildung 3.15 zeigt dabei, dass ein messtechnisch signifikanter Unterschied in den Aktivierungsenergien nur im Bereich des Maximum der Aktivierungsenergie vorliegt, 

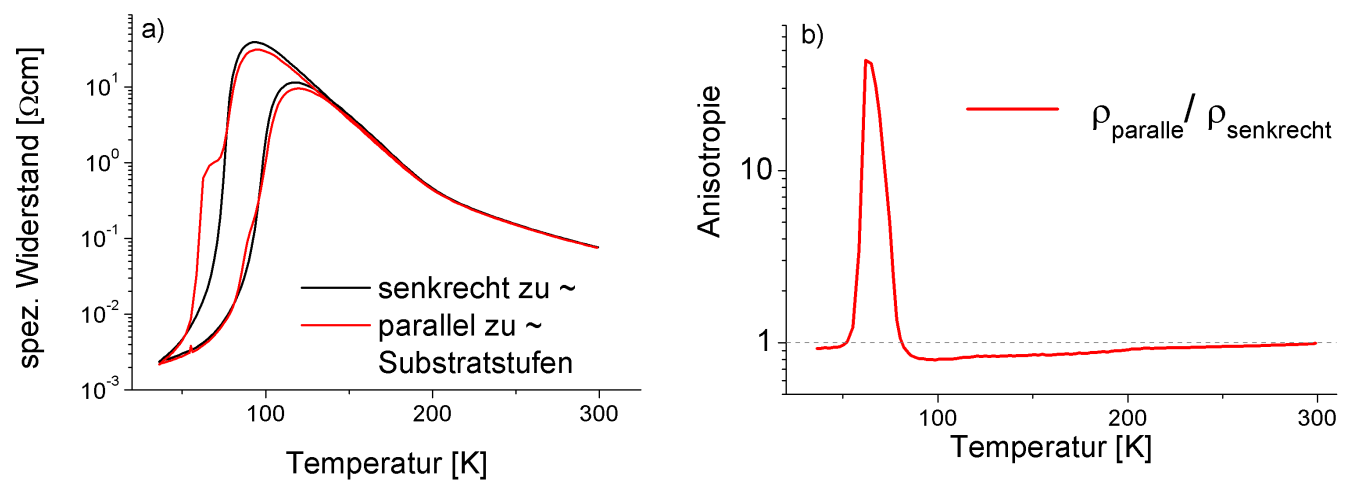

Abbildung 3.14: a) Spezifischer Widerstand über die Temperatur für die Richtung parallel sowie senkrecht zu den Stufen für 9T; b) Daraus bestimmte Anisotropie für die Abkühlkurve; Probe Viz- 33 .

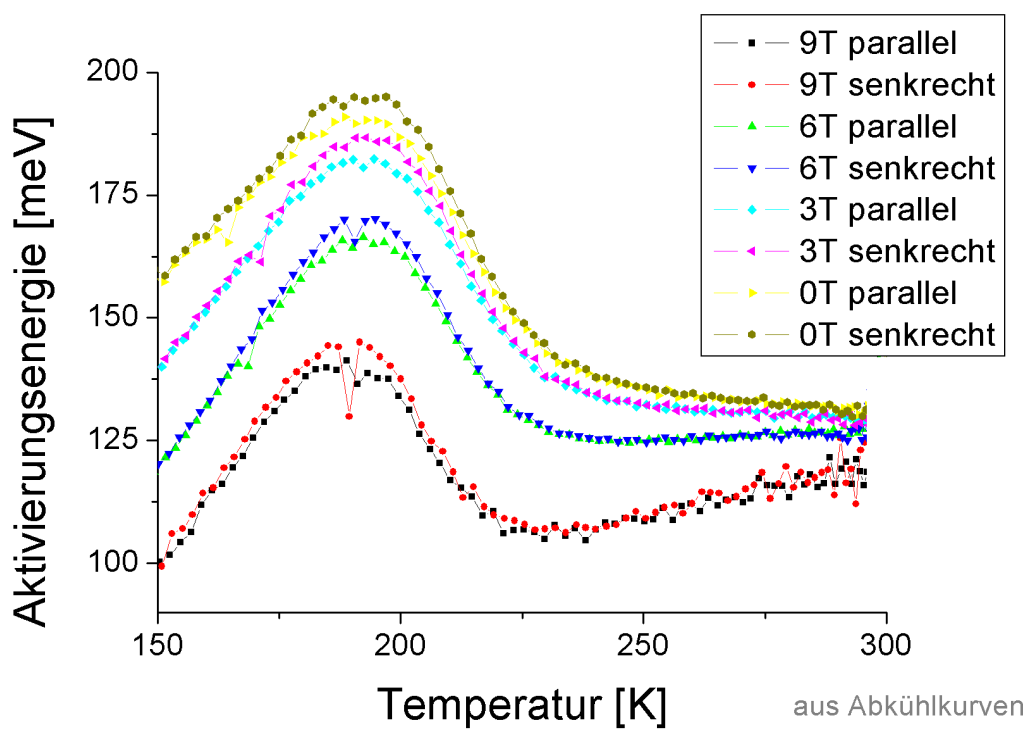

Abbildung 3.15: Aktivierungsenergie über die Temperatur für 0T, 3T, 6T und 9T und beide Messrichtungen (aus der Abkühlkurve bestimmt), Probe ; Probe Viz- $3_{3 h}$.

der allerdings für alle magnetischen Felder zu beobachten ist.

Ähnliche Verhältnisse, also ein Widerstand der parallel zu den Stufen kleiner ist, wird auch bei sehr tiefen Temperaturen beobachtet. Eine Umkehrung der Verhältnisse und damit verbunden eine große Anisotropie wird im Übergangsbereich beobachtet. Die große Anisotropie (vgl. Abb. 3.14 b) ist die Folge eines quasi 
zweistufigen Überganges beim stufenparallelen Widerstand. Die Richtungsaniso-

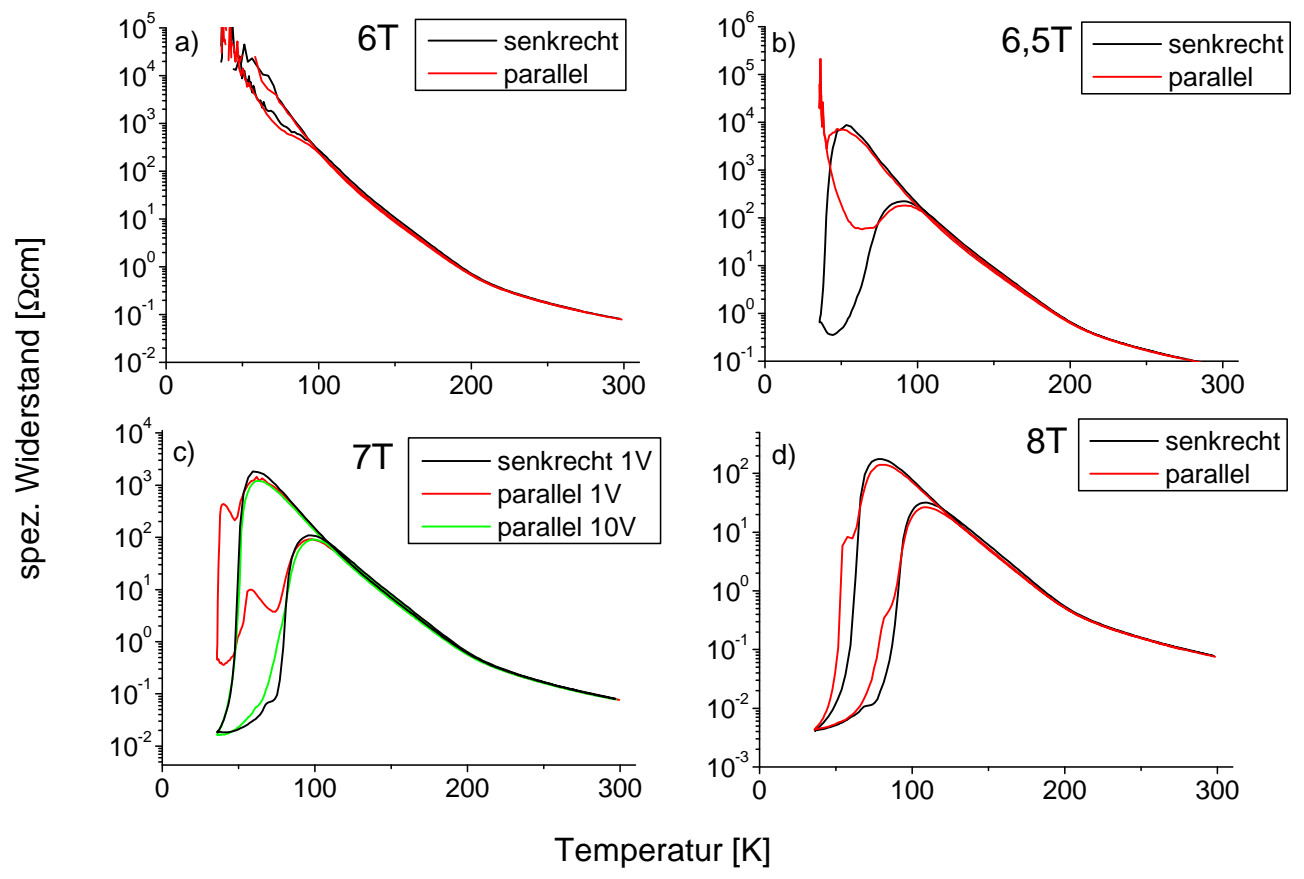

Abbildung 3.16: Spez. Widerstand über die Temperatur im Vergleich für parallele und senkrechte Messrichtung für 6T, 6.5T, 7T und 8T, Messungen mit 1V, nur bei 7T parallel zusätzlich mit $10 \mathrm{~V}$ aufgetragen; Probe Viz- 33 .

tropie zeigt sich verstärkt unterhalb der Übergangstemperatur für mittlere Felder. Für ein Magnetfeld von 6T sind die Unterschiede im Verlauf des Widerstandes über die Temperatur noch sehr klein. Bei einem Magnetfeld von 6,5T zeigt die senkrechte Richtung einen MIT, wohingegen in der parallelen Richtung nur ein lokales Minimum im Widerstand zu sehen ist, der Widerstand steigt aber bei weiterer Temperaturabsenkung bis das Messlimit erreicht ist. In der Aufwärmkurve zeigt sich wieder das hysteretische Verhalten, wobei das Minimum deutlich ausgeprägter ist. Für die senkrechte Richtung steigt im Weiteren die Übergangstemperatur mit ansteigendem Feld, verbunden mit einem immer kleiner werdenden Restwiderstand bei sehr tiefen Temperaturen. Typisch für den Widerstand parallel zu den Stufen ist der zweistufige Übergang, wobei dieser mit zunehmendem Feld immer schwächer ausgeprägt ist. Die Anisotropie weist in diesem Sinne ein Maximum bzgl. des magnetischen Feldes auf.Wie am Anfang dieses Abschnittes gesehen, liegt der erreichte Endwiderstand für die parallele Richtung für 9T wie- 
der unter dem der senktrechten Richtung. Es muss allerdings betont werden, dass diese Ergebnisse Messungen widerspiegeln, bei denen das elektrische Feld bzw. die Stromdichte hinreichend klein waren. Auf die Auswirkungen von elektrischen Spannungen, die in der Abb. 3.16 C mit eingezeichnet sind, soll in folgenden Kapiteln eingegangen werden.

\subsection{Zusammenspiel von magnetischen und elek- trischen Feldern}

\subsubsection{Kinetik der Phasenübergänge}

Bevor man sich den komplexen Abhängigkeiten des Widerstandes von der Kombination von elektrischen und magnetischen Feldern widmet, muss man klären, welche Rolle die Zeitabhängigkeit spielt. Für endliche Abkühlgeschwindigkeiten (möglichst langsam) kommt es zu einer Überlagerung der Temperaturabhängigkeit des Widerstandes und z. B. der strukturellen Relaxation in phasenseparierten Systemen. Bei schneller Abkühlung kann es zu einer technischen Temperaturhysterese dadurch kommen, dass das Thermoelement keine optimale thermische Kopplung an die Probe aufweist. Des Weiteren können endliche Pulslängen zur Vermeidung von Aufwärmungen dazu führen, dass die benötigte Zeit, wie sie z. B. für eine Response das CER-Effektes benötigt wird [80], nicht vorhanden ist. In Abbildung 3.17 links ist der Widerstand für verschiedene Abkühlgeschwindigkeiten über die Temperatur aufgetragen. Die Temperaturschleifen (300K - 10K - 300K, alle bei 9T) wurden mit immer langsameren Abkühlgeschwindigkeiten gefahren. Die Wartezeit im Nullfeld zwischen den Schleifen beträgt für die ersten drei Schleifen 10 min, vor der letzten Schleife wurde 1 Tag die Probe bei 300K und $0 \mathrm{~T}$ belassen. Wie am Einsatz a1 der Abb. 3.17 zu erkennen ist, reichten 10 min für eine Relaxation der Probe auf immer den gleichen Anfangswiderstand nicht ganz aus, wohingegen nach einem Tag der Raumtemperaturwiderstand fast den Wert vor den Messungen entspricht. Der erreichte Endwiderstand (Abb. 3.17 Einsatz a2) spiegelt sich in der relativen Höhe des Anfangswiderstandes wieder, wohingegen die Abkühlgeschwindigkeit keine Rolle spielt. Die zu erkennende Temperaturhysterese ist kein messtechnisches Artefakt, sondern stammt vom Transportverhalten der Probe. Dies ist besonders gut daran zu erkennen, dass sich die Breite der Hysterese nicht mit der Abkühlgeschwindigkeit ändert, sowie das oberhalb von $100 \mathrm{~K}$ die Kurven nahezu übereinander liegen. Die thermische Kopplung von Thermoelement und Probe ist somit optimal gegeben. Betrachtet man die Zeitabhängigkeit der Leitfähigkeit auf sehr kurzer Zeitskala, so findet man über den gesamten Magnetfeldbereich im messtechnisch relevanten Bereich einen zeitunabhängigen Widerstand (Abb. 3.17 rechts). Dies ändert sich, wenn man den CMR-Übergangsbereich näher untersucht, welches später dargestellt 

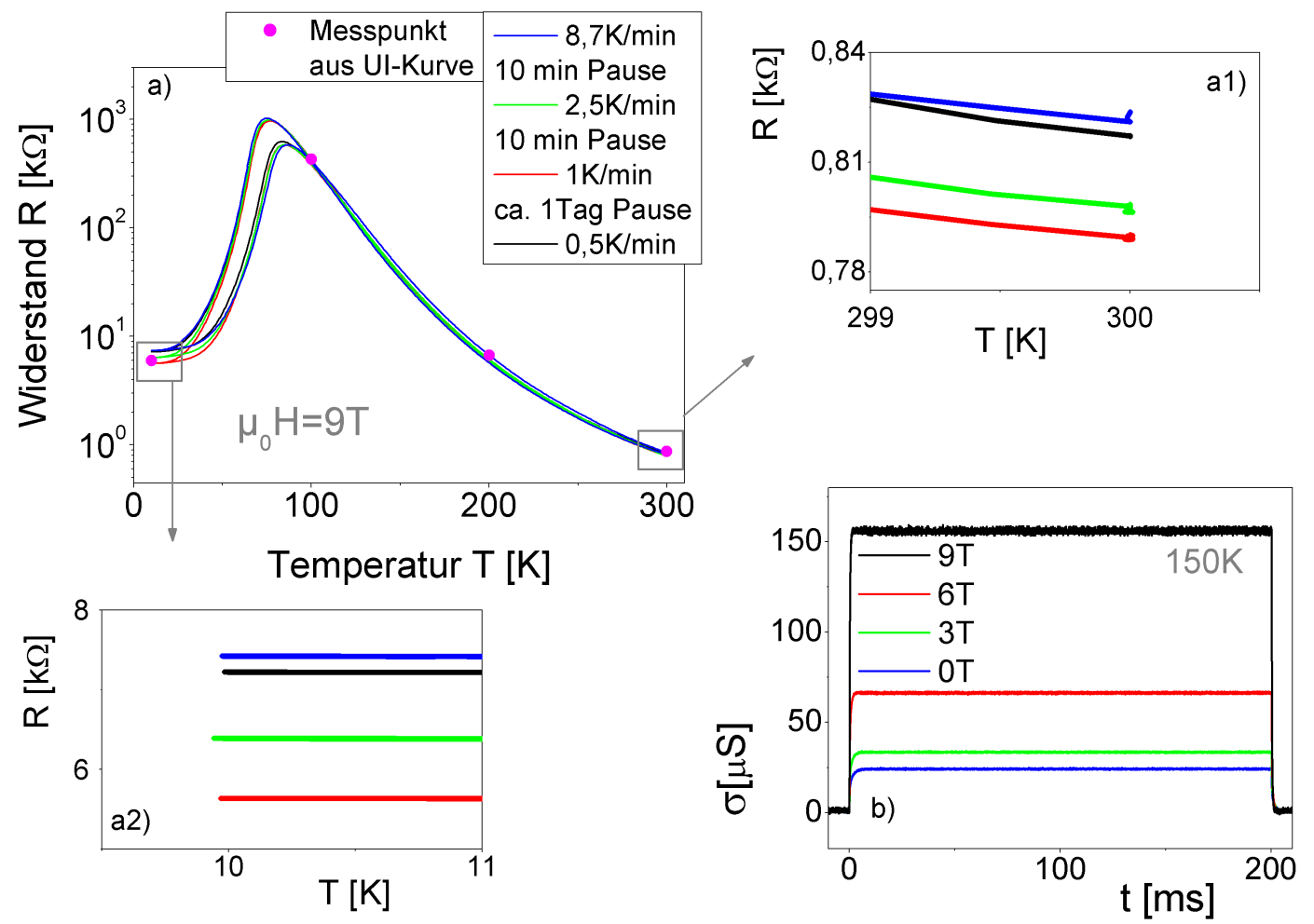

Abbildung 3.17: links: Widerstand über Temperatur für unterschiedliche Abkühlgeschwindigkeiten in einem Magnetfeld von 9T, gesputterte Probe mit typischem CMREffekt von PCMO; Einsatz: Vergrößerungen für 300K sowie 10K, rechts: zeitliches Transportverhalten für 0T, 3T, $6 \mathrm{~K}$ sowie $9 \mathrm{~T}$ bei $150 \mathrm{~K}$, Probe Viz- $3_{3 h}$.

wird. Ebenfalls sehr bemerkenswert ist die Wegunabhängigkeit. Unabhängig vom Messmodus erhält man vergleichbare Widerstände. In Abbildung 3.17 links sind neben den feldgekühlten Temperaturschleifen auch Messpunkte eingezeichnet, bei denen im Nullfeld die relevante Temperatur angefahren wurde und dann isotherm das Magnetfeld auf die gleiche Stärke (9T) erhöht wurde.

\subsubsection{Abhängigkeit des Widerstandes vom elektrischen Feld}

Im elektrischen Feld sind zwei Effekte in PCMO zu unterscheiden:

- Die Abhängigkeit der polaronischen Mobilität vom elektrischen Feld durch die Absenkung der Aktivierungsbarriere (Abb. 3.13) [5];

- Der Kolossale Elektrowiderstandseffekt (CER-Effekt) als eine sprunghafte 
Änderung im Widerstand, der mit einem Ordnungs-Unordnungsübergang verbunden ist [42].

In Abb. 3.18 a) sind aus [79] CER-Effekt Kurven für verschiedene Stromdichten zu sehen. Für kleine Ströme findet sich der gewohnte isolierende Verlauf. Mit
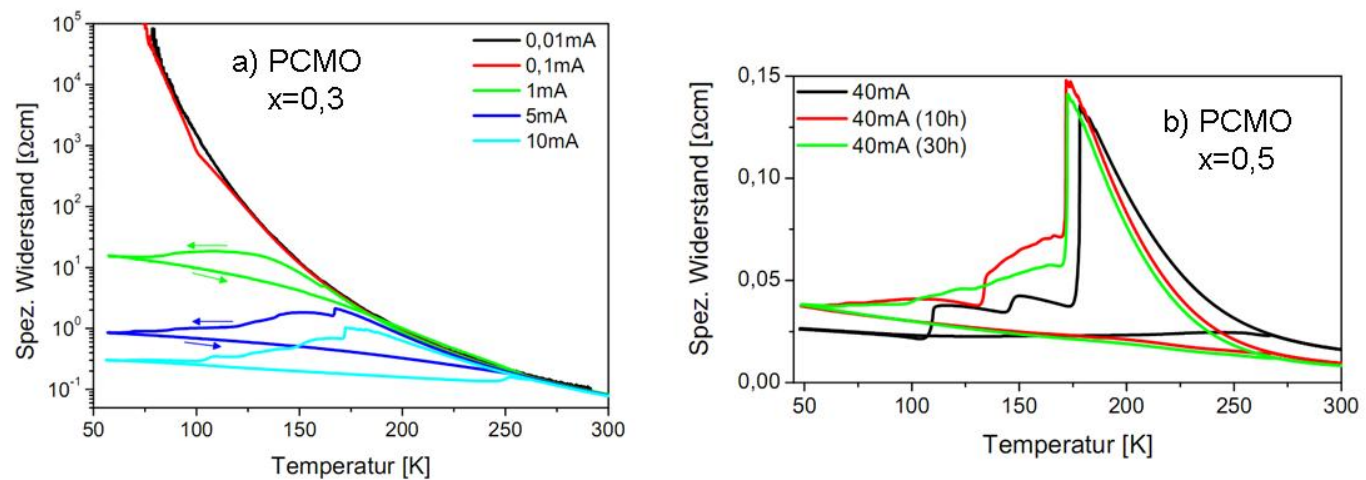

Abbildung 3.18: Messungen aus [79]; Widerstandsverhalten im CER-Effekt für a: unterschiedliche Stromdichten und b: Auslagerungszeiten.

steigender Stromdichte kommt es zum CER-Effekt, der Widerstand ist um viele Größenordnungen verringert. Durch das dauerhafte Anliegen des elektrischen Stromes wird die Ausbildung der geordneten Phase unterdrückt. Da Defekte hauptsächlich die Ausbildung der geordneten Phase negativ beeinflussen, diese aber im CER-Effekt bereits unterdrückt ist, sollte es nahezu keine Abhängigkeit des CER-Effekt von Defekten geben. Die Abhängigkeit von Defekten, wieder realisiert über eine thermische Nachbehandlung, ist in Abb. 3.18 b) dargestellt. Wie zu erwarten, ist zwar eine Absenkung des Raumtemperaturwiderstandes, aber keine dramatische Änderungen im Widerstandsverlauf über die Auslagerungszeit zu finden.

Eine Anpassung der temperaturabhängigen Leitfähigkeit im CER-Effekt an das TAP-Modell ist auch gegeben, soll aber im Rahmen dieser Arbeit nicht diskutiert werden. Im Folgenden soll das isotherme Transportverhalten näher beleuchtet werden, um den Einfluss des elektrischen Feldes zu untersuchen. Dafür sind in Abb. 3.19 a) das Widerstandsverhalten für verschiedene Temperaturen gegen den Strom aufgetragen. Betrachtet man das Widerstandsverhalten oberhalb von 200K, so ist ein Übergang vom ohm'schen in das nichtohm'sche Verhalten (mit 1 gekennzeichnet) zu finden. Dieses Verhalten erwartet man nach dem Modell der thermisch aktivierten Polaronen (TAP-Modell) (2.3.1). Wenn man den spezifischen Widerstand $\rho$ auf $\rho_{0}$ normiert und als Maß für das kritische elektrische Feld 

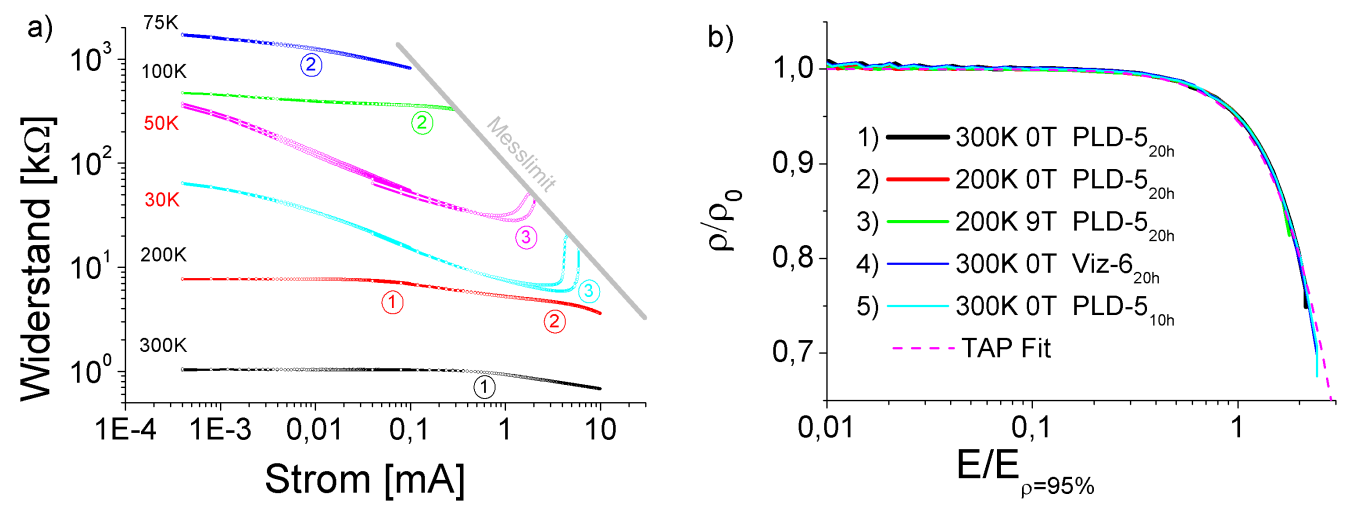

Abbildung 3.19: a: typische Widerstand über Strom-Kennlinien für verschiedene Temperaturen bei $9 \mathrm{~T}$, Probe Spu- $2_{a p}$; b: Normierte Widerstandskurven über ein elektrisches Überschneidungsfeld mit eingezeichneter theoretisch nach dem TAP-Modell zu erwartender Kurve. Anpassparameter und Probendaten können Tabelle 3.1 entnommen werden.

$E_{c}$ das elektrische Feld $E_{\rho=95 \%}$ nimmt, das man benötigt, um den spezifischen Widerstand um 5\% abzusenken, so fallen alle Kurven auf die nach dem TAP-Modell erwartete Kurve (Abb. 3.19 b). Dies gilt sogar für den gesamten untersuchten Magnetfeldbereich bis 9T, sowie für unterschiedliche Auslagerungszeiten. Bei tiefen

\begin{tabular}{l|l|l|l} 
Kurve & $E_{c}[\mathrm{~V}]$ & $\rho_{0}[\Omega c m]$ & Probe \\
\hline 1 & 29,3 & 0,041 & PLD-5 $20 h$ \\
\hline 2 & 61,3 & 0,1404 & PLD- $5_{20 h}$ \\
\hline 3 & 56 & 0,2669 & PLD-5 $5_{20 h}$ \\
\hline 4 & 31,3 & 0,0471 & Viz-6 $20 h$ \\
\hline 5 & 31,2 & 0,053 & PLD- $5_{10 h}$
\end{tabular}

Tabelle 3.1: Für die Anpassung an das TAP-Modell erhaltene Parameter in Anlehnung an Abb. 3.19.

Temperaturen ist des TAP-Modell streng genommen nicht mehr anwendbar, es lassen sich aber analoge Übergänge vom ohm'schen zu nichtohm'schen Verhalten finden, wobei für tiefe Temperaturen kleinere Felder/ Ströme nötig sind. Es treten aber zwei weitere Änderungen des Widerstandsverhaltens auf. In Abb. 3.19 a) ist mit (2) eine weitere Absenkung des Widerstandes gekennzeichnet, die mit dem CER-Effekt in Verbindung gebracht werden könnte, wobei diese Absenkung im 
Gegensatz zum konventionellen CER stetig ist. In gleicher Abb. mit (3) gekennzeichnet ist ein Anstieg im Widerstandsverlauf bei hohen elektrischen Feldern bzw. Strömen zu sehen, der nicht auf Erwärmung zurück zu führen ist. Dieser Anstieg wurde nur in Messungen gefunden, wo sich die Probe im CMR-Effekt und damit gut leitfähigen Tieftemperaturzustand befand. Die Kombination von magnetischen und elektrischen Feldern (die einzeln betrachtet den Widerstand absenken) führt für steigende elektrische Felder zu einem Anstieg des Widerstandes. Das nicht einfache Wechselspiel dieser beiden Felder wird im Folgenden genauer diskutiert.

\subsubsection{Elektrische Felder im Übergangsbereich}

In diesem Abschnitt wird gezeigt, dass ein Übergang von der isolierenden Tieftemperatur-Phase in die ferromagnetisch-metallartige Phase in einem Magnetfeld vorbereiteten Zustand durch ein zusätzliches starkes elektrisches Feld möglich ist. Ebenso kann ein starkes elektrisches Feld hohe Ströme induzieren, die diesen Übergang umkehren können.

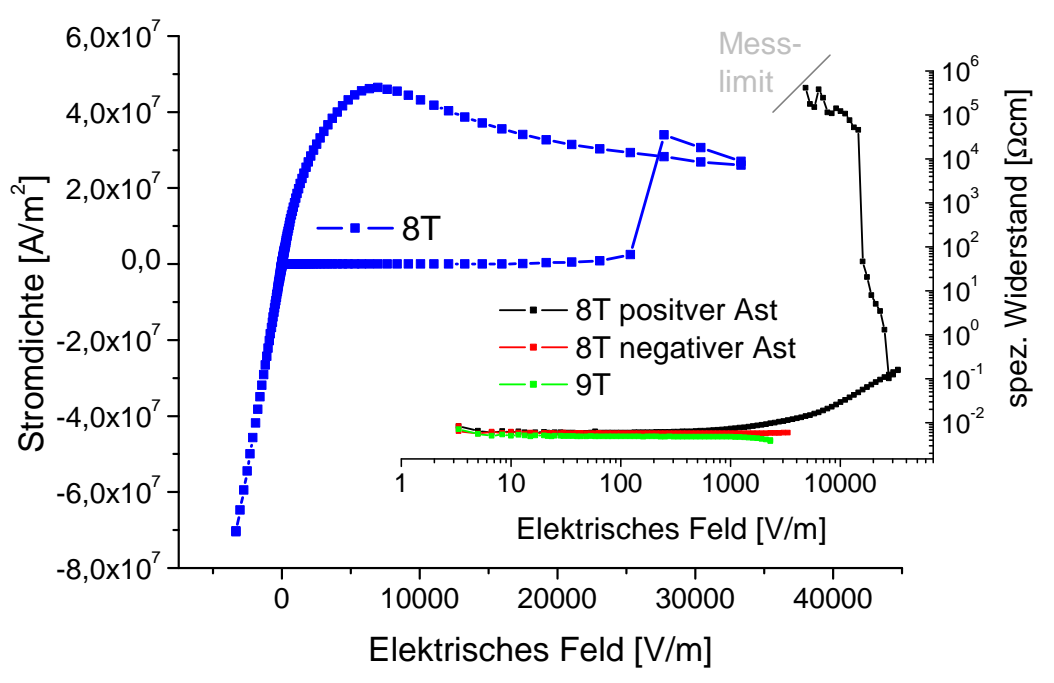

Abbildung 3.20: außen: Stromdichte über elektrisches Feld bei 8T, innen: spez. Widerstand über elektrisches Feld bei 8T und 9T der selben Messung wie außen; Temperatur 10K; Probe Viz- 3 .

Durch eine geeignete Wahl von Temperatur und magnetischem Feld lassen sich nun Zustände realisieren, in denen die Leitfähigkeit extrem sensibel und irreversibel auf Änderungen des elektrischen Feldes reagiert. In diesem Sinne stellen 
solche Zustände metastabile Zustände dar, die im Übergangsbereich zwischen der ferromagnetisch-metallartigen Phase und der antiferromagnetisch-ladungsgeordneten Phase auftreten.

Ein Beispiel dafür zeigt Abb. 3.20. Hier wurde nach einer Nullfeldabkühlung bei $10 \mathrm{~K}$ ein Magnetfeld von 8T angelegt, bei einem Feld von 8,4T würde bei dieser Temperatur ein Übergang in den gut leitfähigen Zustand erfolgen. Für kleine Messspannungen verbleibt die Probe dementsprechend im hochisolierenden $\mathrm{Zu}$ stand. Beim Anlegen eines Feldes von ca. $25 \mathrm{kV} / \mathrm{m}$ erfolgt ein Übergang in den niederohmigen Zustand (ferromagnetisch-metallartige Phase). Der Widerstand bricht um über vier Größenordnungen ein. Bei weiterer Stromerhöhung kommt es zu einem Anstieg des Widerstandes. Dieser durch das elektrische Feld induzierte Übergang ist irreversibel, dass heißt die Probe verbleibt nach Abschalten des elektrischen Feldes im niederohmigen Zustand (die U(I)-Kennlinie wird jetzt reversibel durchlaufen, vgl. „negativer Ast“ in Abb. 3.20). In diesem Sinne stellt der geschilderte Effekt einen elektrisch induzierten isothermen CMR-Übergang dar. Eine weitere bemerkenswerte Eigenschaft dieses Effektes ist, dass er nicht den Charakter einer reinen Instabilität hat, sondern dass es möglich ist, eine Reihe von zeitlich relativ stabilen Zwischenzuständen zu realisieren. Die Abb. 3.21 zeigt

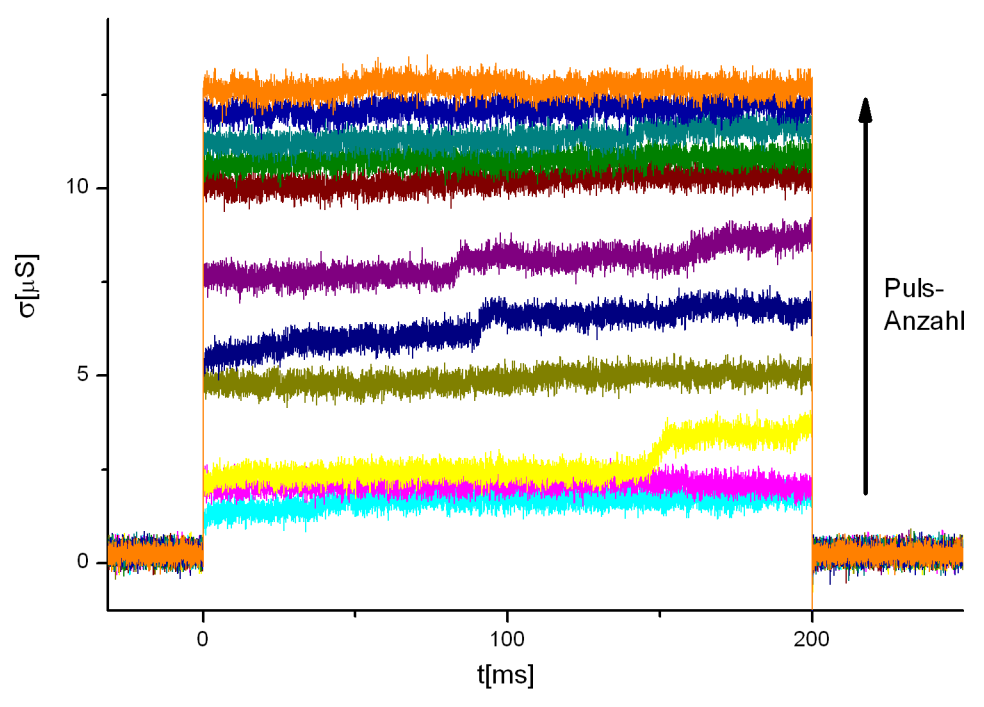

Abbildung 3.21: Leitfähigkeit über die Zeit für aufeinander folgende Messungen mit einer Länge von $200 \mathrm{~ms}$ und einer Spannung von $2 \mathrm{~V}$ bei $30 \mathrm{~K}$ und $7 \mathrm{~T}$; Probe Viz- $3_{3 h}$.

die Leitfähigkeit für eine Folge von zeitlich aufeinander folgenden Spannungspulsen gleicher Länge und Amplitude. Die Leitfähigkeitsantwort der Probe stellt sich in den Grenzen der zeitlichen Auflösung (ca. 1ms) quasi instantan ein. Es treten während des Pulses noch weitere sprunghafte aber auch stetige Erhöhungen der Leitfähigkeit auf. Diese während des Pulses induzierte Leitfähigkeitserhöhung ist 
remanent, d.h. sie bleibt nach Abschalten des elektrischen Feldes erhalten. Ein weiterer Puls erhöht die Leitfähigkeit weiter, sodass in der akkumulierten Wirkung der Pulssequenz eine hohe Leitfähigkeit erreicht wird. Diese Experimente implizieren, dass der Übergang zwischen dem isolierenden und dem gut leitfähigem Zustand eng mit der Mobilität der polaronischen Ladungsträger verknüpft ist. Dies wird auch durch die Existenz eines inversen Effektes verdeutlicht. Er basiert auf der elektrischen Remanenz, die man bei tiefen Temperaturen beobachtet. Eine durch das Magnetfeld induzierte gute Leitfähigkeit bleibt erhalten, wenn man das magnetische Feld abschaltet. Die Abb. 3.22 zeigt zunächst noch-

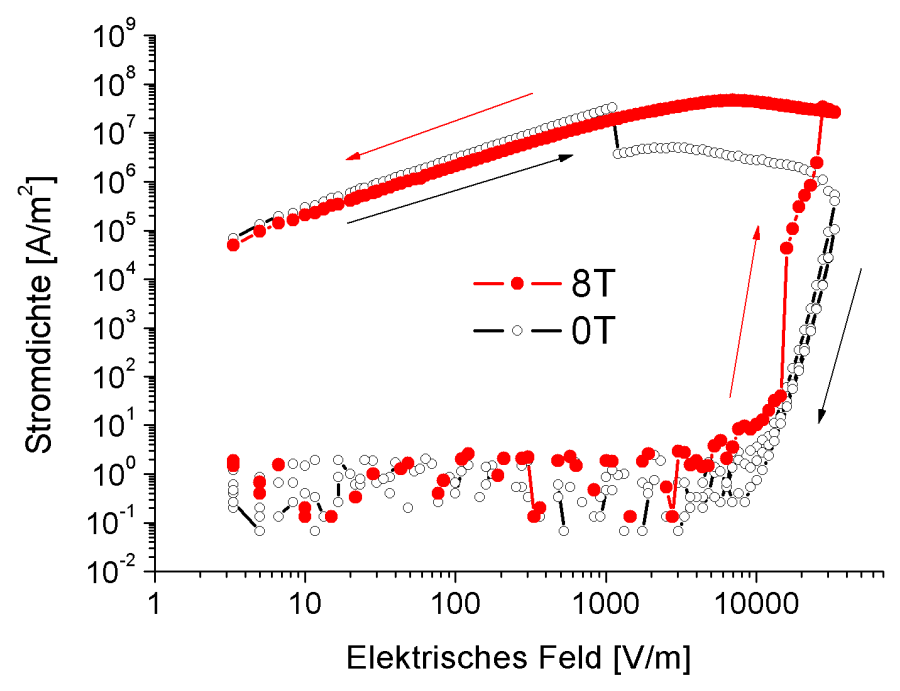

Abbildung 3.22: Stromdichte über elektrisches Feld für 8T mit irreversibler Änderung der Leitfähigkeit sowie der späteren Messung bei 0T; gemessen bei 10K; Probe Viz- $33 h$.

mals, wie ein Übergang in den gut leitfähigen Zustand im Magnetfeld durch das elektrische Feld unterstützend induziert wird. Diese hohe Leitfähigkeit bleibt auch erhalten, wenn man sowohl das elektrische als auch das magnetische Feld auf Null setzt. Ein erneutes Durchlaufen der Strom-Spannungs-Kennlinie führt nun ohne Magnetfeld zu einem irreversiblen Übergang in den isolierenden Zustand.

\subsubsection{Remanenz der elektrisch leitfähigen Phase}

In diesem Abschnitt sollen Eigenschaften der remanenten elektrisch leitfähigen Phase untersucht werden, insbesondere ihre Stabilität als Funktion der magnetischen Vorgeschichte und der angelegten elektrischen Felder.

Die remanente gute Leitfähigkeit bei tiefen Temperaturen ist eine Temperatur- 
und zeitabhängige Größe [56, 81, 83, 97]. Man kann die elektrisch leitfähige Remanenz dazu nutzen, mehr über die Eigenschaften der ferromagnetisch-metallartigen Phase zu lernen. Metastabile Zwischenzustände können in der Remanenz beim Übergang geordnete Phase/ ferromagnetisch-metallartigen Phase eingestellt werden.

In Abbildung 3.23 sind als Beispiel für die elektrisch leitfähige Remanenz eine Nullfeld-Abkühlkurve sowie eine Abkühlkurve im Magnetfeld gezeigt. Wird die

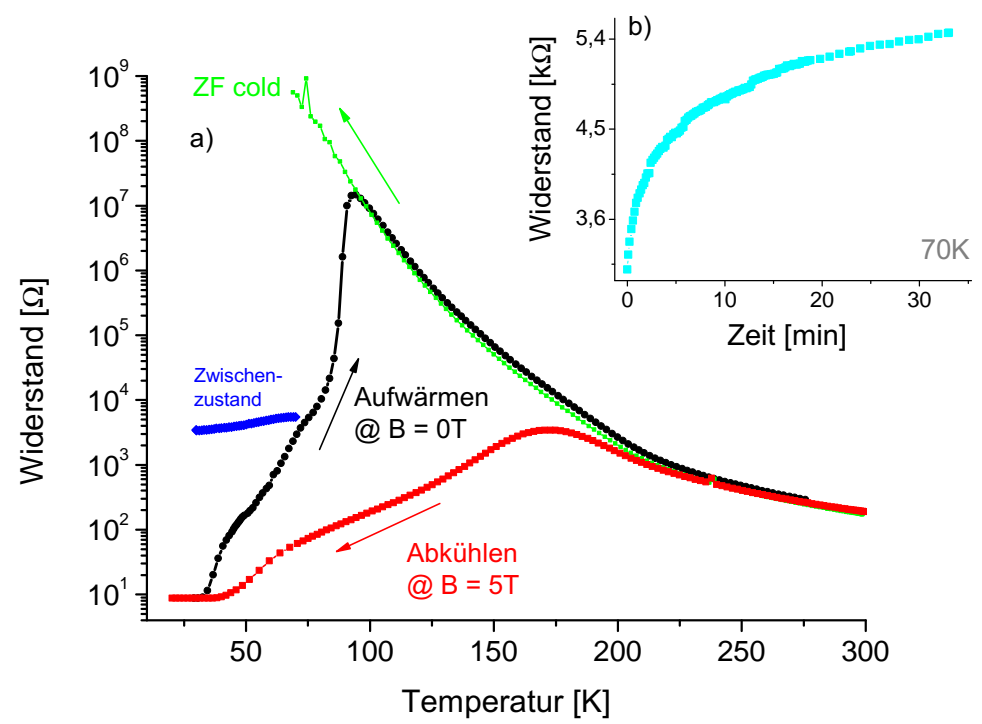

Abbildung 3.23: Widerstand über der Temperatur Nullfeldkurve sowie Messung mit 9T, dazwischen die Aufwärmkurve für die elektrische Remanenz im Nullfeld, sowie Messung im Zwischenzustand (Erklärung siehe Text); im Einsatz zeitliche Relaxation des Widerstandes bei einem Zwischenstopp bei 70K; alle Messungen dieser Abbildung wurden mit sehr kleinen elektrischen Feldern (kleiner $500 \mathrm{~V} / \mathrm{m}$ ) und somit auch Stromdichten gemacht; Probe PLD-1 $1_{80 h}$.

Probe im Nullfeld bis 10K abgekühlt, ist sie hoch isolierend. Wird nun bei dieser Temperatur ein Magnetfeld hinreichender Größe angelegt (für die Probe PLD-1 5T) und dann wieder abgeschaltet, zeigt sie den gleichen Widerstand wie eine im Magnetfeld abgekühlte Probe, bei der erst bei 10K das Magnetfeld abgeschaltet wurde. Wärmt man nun die Probe aus dem elektrisch remanenten Zustand wieder auf, so erhält man erst ab $35 \mathrm{~K} \pm 3 \mathrm{~K}$ einen signifikanten Anstieg im Widerstand im Vergleich zur Aufwärmkurve im Magnetfeld. Diese Temperatur hängt weder von der speziellen Probe noch von der Auslagerungszeit ab. Bei 95K geht die „remanente Kurve“ für diese Probe wieder in die Nullfeld-Kurve über. Verbleibt 
die Probe bei einer Temperatur von 10K nach der Überführung in den elektrisch leitfähigen remanenten Zustand, so ist der Widerstand über Stunden konstant. Oberhalb der charakteristischen Temperatur von 35K treten aber deutliche Relaxationseffekte auf. Dies zeigt der Einsatz von Abb. 3.23 bei einer Temperatur von $70 \mathrm{~K}$. Erniedrigt man ohne Magnetfeld die Temperatur wieder auf 30K, so liegt der Widerstand zwar niedriger als bei 70K, aber um Größenordnungen höher als der Widerstand im Feld. Die anschließende Aufwärmung (beschrieben mit „Zwischenzustand“ in Abb. 3.23) auf 70K führt zu einem Widerstand, der den selben Wert wie das direkte Aufwärmen aus dem remanenten Zustand hatte.

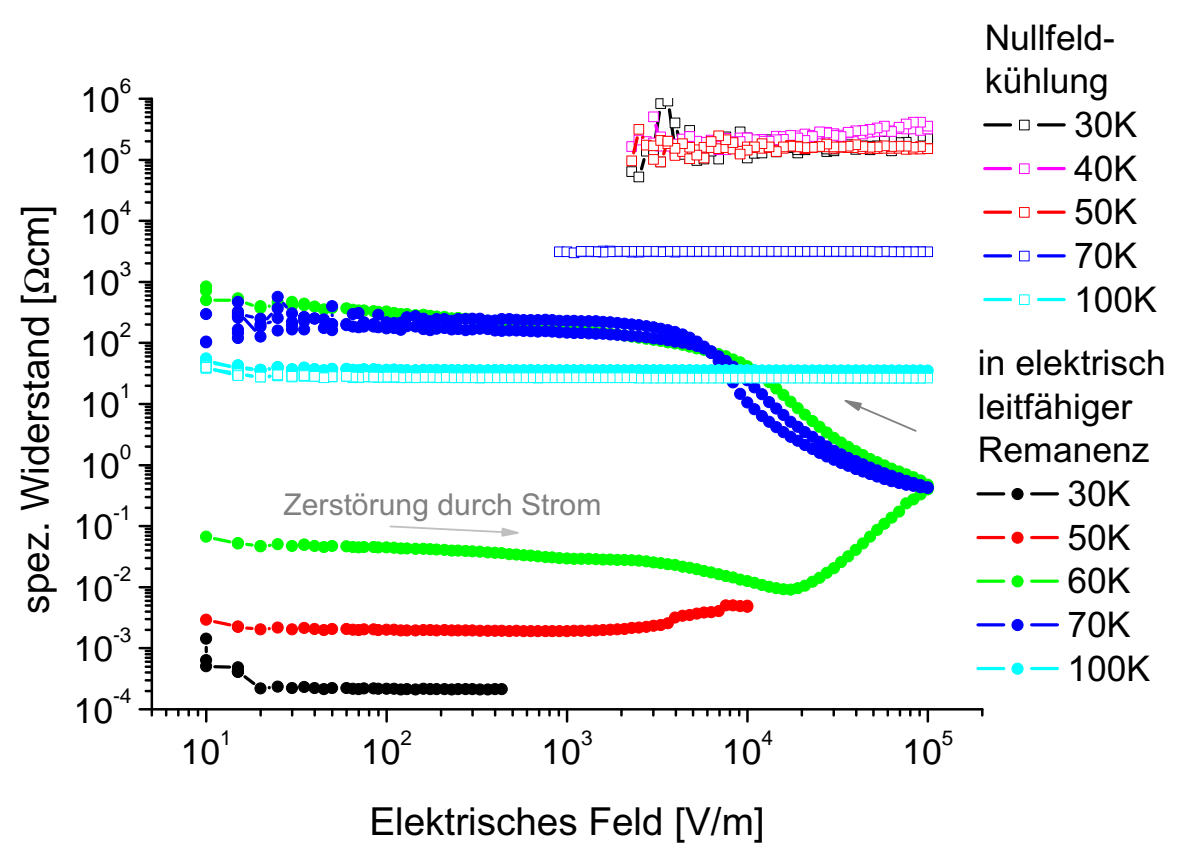

Abbildung 3.24: Spezifischer Widerstand über Spannung für verschiedene Temperaturen einmal nach Nullfeldkühlung innerhalb einer Rampe, sowie während des Aufwärmens aus der remanenten Phase ebenfalls im Nullfeld innerhalb einer Rampe; Probe PLD- $1_{80 h}$.

Abbildung 3.24 fasst nochmals die wichtigsten Eigenschaften der elektrischen Remanenz zusammen. Aufgetragen ist der spezifische Widerstand gegen das elektrische Feld. Die oberen Grenzen der jeweiligen angelegten Spannungen sind in Folge der Leistungsbegrenzung, um eine Erwärmung der Proben zu vermeiden. Kühlt man im Nullfeld auf die jeweilige Temperatur ab (offene Symbole), so liegt in den messtechnisch zugänglichen Bereichen ein ohmscher Widerstand vor. Erwärmt 
man die Probe aus dem remanenten Zustand (geschlossene Symbole) auf die korrespondierende Temperatur, so sind die entsprechenden ohmschen Widerstände um Größenordnungen kleiner und die Strom-Spannungs-Kennlinien hysteretisch und nichtlinear. Bei einer Temperatur von $100 \mathrm{~K}$ verschwinden die Unterschiede zwischen Nullfeldkühlung und Erwärmung aus dem remanenten Zustand. Die hysteretischen U-I-Kennlinien führen in der Regel den gut leitenden Zustand nicht in den korrespondieren Zustand, d.h. der Widerstand ist nach Durchlaufen der Hysterese immer noch deutlich kleiner als im Zustand der Nullfeldkühlung. Die Systematik in der Abb. 3.24 legt aber nahe, dass dies bei sehr hohen elektrischen Feldern möglich ist, solche Spannungen sind messtechnisch durch die resultierenden Probenerwärmungen aber nicht möglich. In Abbildung 3.24 sind für verschiedene Temperaturen im remanenten leitfähigen Zustand sowie für Nullfeldkühlungen Kurven für spezifische Widerstände über die angelegte Spannung aufgetragen. Für die im Nullfeld aufgenommenen Kurven ist über den gesamten Bereich ohmsches Verhalten gut zu erkennen. Die Kurve für 30K im remanenten Zustand zeigt bis zum Messabbruch (Schutz vor Zerstörung der Probe durch zu hohe Ströme) ebenso ohmsches Verhalten. Bei 50K ist für höhere Spannungen ein Anstieg im Widerstand zu erkennen. Bei einer Temperatur von $60 \mathrm{~K}$ ist bei steigendem elektrischen Feld noch dessen begünstigende Wirkung zu sehen, aber ab einem kritischen Strom kommt es auch hier zum Anstieg des Widerstandes und sogar zu einem negativen differentiellen Widerstand, Abb. 3.25.

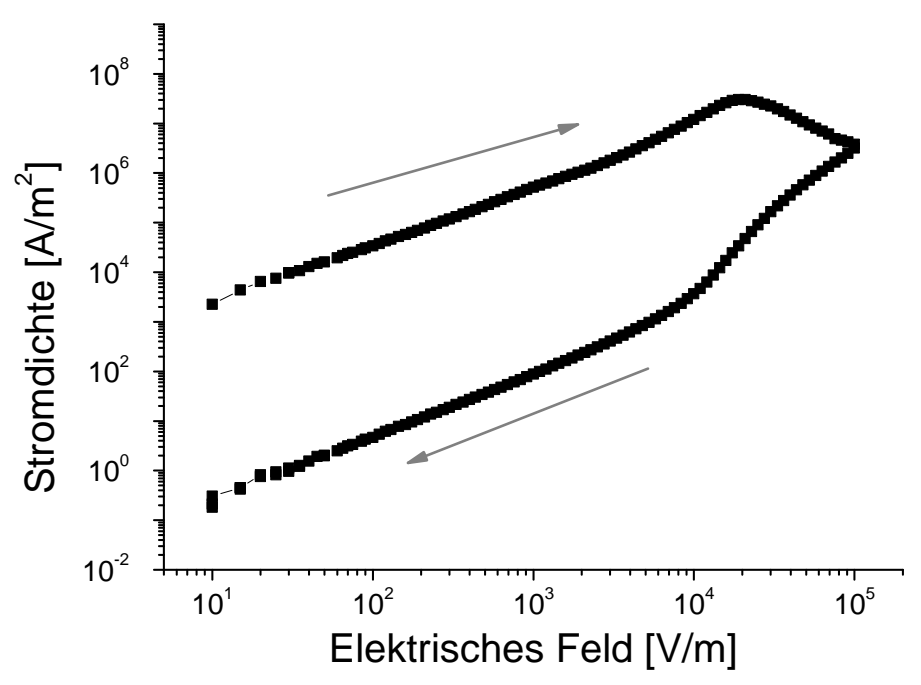

Abbildung 3.25: Strom-Spannungs-Kurve (60 K, aus der remanenten Phase) zur Messung in Abb. 3.24 gehörend; Probe PLD- $1_{80 h}$. 
Im Unterschied zur Probe in Abb. 3.22 ist diese länger ausgelagert. Der Übergang in den niederohmigen Zustand erfolgt nicht sprunghaft. An der darauf folgenden Kurve bei $70 \mathrm{~K}$ ist am besten zu erkennen, dass die elektrische Remanenz nicht vollständig zerstört wurde. Der Widerstand ist noch nicht auf den der Nullfeldkurve zurück gefallen. Mehr noch, mit steigendem elektrischen Feld wird der Widerstand feldabhängig erniedrigt.

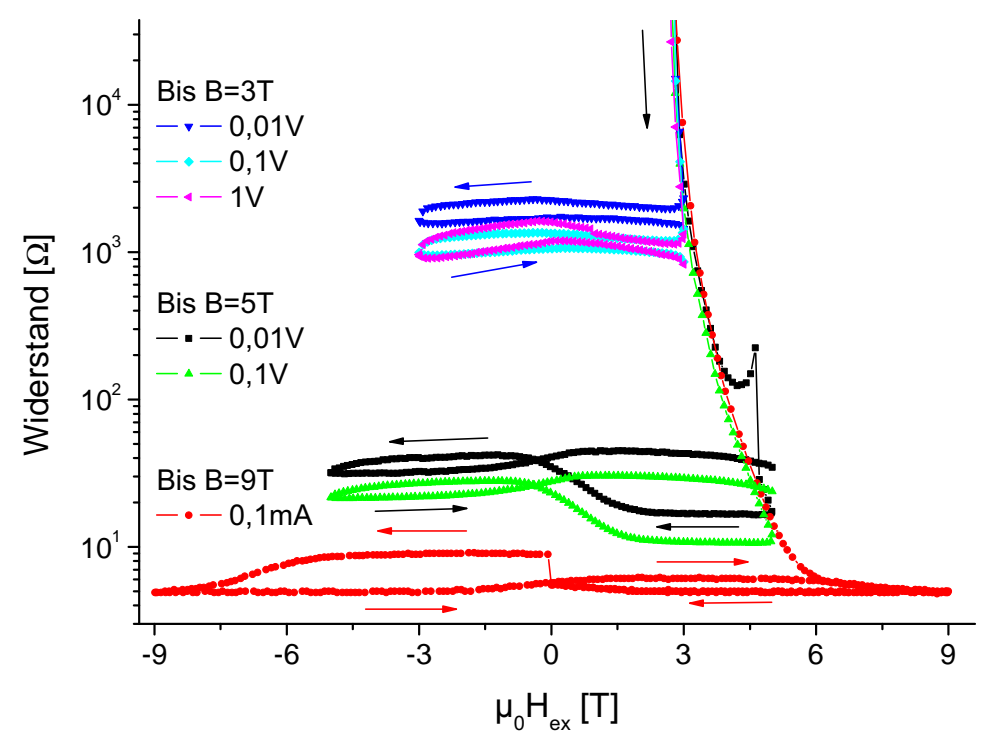

Abbildung 3.26: Widerstand über angelegtes Magnetfeld bei 30K, Schleifen für 3T, 5T, 9T Maximalmagnetfeld, 3T und 5T mit unterschiedlichen Messspannungen, 9T mit kleinem Messstrom; Probe PLD-1 $1_{80 h}$.

Abbildung 3.26 zeigt nun die Magnetfeldabhängigkeit der elektrisch leitfähigen Remanenz. Alle Messungen sind nach einer Nullfeldkühlung von Raumtemperatur auf $30 \mathrm{~K}$ durchgeführt worden. Ausgehend vom Nullfeldzustand bricht ab 2,5T der Widerstand $\left.\left(R(0 T)>10^{11} \Omega\right)\right)$ exponentiell ein. Bei verschiedenen Magnetfeldern wurden jetzt Feldschleifen bei unterschiedlichen elektrischen Feldern aufgenommen. Eine Sättigung in der Form, dass die Leitfähigkeit trotz eines steigenden Magnetfeldes nicht merklich weiter ansteigt, tritt ab 6,4T ein. Betrachtet man zunächst die Feldschleife bei 9T, so ist der Widerstand nahezu feldunabhängig. Allerdings sind Relaxationseffekte bei 30K schon merklich, der Sprung im Widerstand bei 0T ist die Folge einer ca. 22 minütigen Wartezeit. Allerdings wird diese Relaxation durch ein erneutes Durchlaufen der Feldschleife (linker Ast in Abb. 3.26) wieder rückgängig gemacht. In diesem Sinne ist der remanente Widerstand eindeutig nur durch das aufmagnetisierende maximale Feld bestimmt. 
Dass dies nicht allgemein gilt, zeigt der Vergleich mit den Feldschleifen bis 5T und 3T. Bei der 5T-Schleife ist der remanente Widerstand im Nullfeld nach Durchlaufen der Hystereseschleife zwar wieder erreicht, das erste Anfahren des maximalen Feldes ist aber mit einer sprunghaften Widerstandsänderung (lokales Maximum in der schwarzen Kurve) verbunden und der resultierende Widerstand wird beim erneuten Durchlaufen der Hysterese nicht mehr erreicht. Bei der 3T-Feldschleife ist der remanente Widerstand im Nullfeld nicht mehr eindeutig, sondern vorgeschichtsabhängig. Diese Systematik ist nicht wesentlich durch das elektrische Feld beeinflusst. Höhere elektrische Felder führen zwar zu niedrigeren Widerständen aber vergleichbaren Hysteresekurven.

\subsubsection{Vizinale Filme}

Im Abschnitt 3.3 wurde schon auf die besonderen Transporteigenschaften von Proben auf vizinalen Substraten verwiesen. Bei der dort diskutierten Probe mit vergleichsweise niedrigen charakteristischen Temperaturen für den CMR (93K bei 9T) waren die Widerstände parallel und senkrecht zu den Substratstufen bis zu Temperaturen von ca. 70K ziemlich identisch. Die Abb. 3.27 a und b verdeutlichen dies nochmals anhand der feldabhängigen Widerstände. Oberhalb der Übergangstemperatur (z. B. $110 \mathrm{~K}$ in Abb. 3.27 a) ist über den gesamten experimentell zugänglichen Feldbereich der stufenparallele Widerstand etwas kleiner. Unterhalb der Übergangstemperatur (z. B. 70K in Abb. 3.27 b) findet man über einen weiten Feldbereich des hysteretischen feldabhängigen Widerstandes das gleiche Verhältnis. Bei sehr hohen Feldern wird aber der stufensenkrechte Widerstand signifikant kleiner als der stufenparallele Widerstand. Im Abschnitt 3.3 wurde aber schon darauf hingewiesen, dass der Übergang in den leitfähigen Zustand in der stufenparallelen Richtung zweistufig erfolgt. Dieses zweistufige Verhalten wird auch im feldabhängigen Widerstand bei einer festen Temperatur (hier $50 \mathrm{~K}$ in Abb. $3.27 \mathrm{c}$ ) beobachtet. Es zeigt sich auch, dass dieser zweistufige Übergang durch hinreichend hohe elektrische Felder unterdrückt werden kann, wobei zu der Temperatur von 50K hier ein Feld von 7,5T korrespondiert, was den Einsatzpunkt der vom elektrischen Feld abhängigen Zweistufigkeit kennzeichnet. Des Weiteren ist noch bemerkenswert, dass die Probe mit ihrer vergleichsweise niedrigen Übergangstemperatur von $93 \mathrm{~K}$ bei $50 \mathrm{~K}$ keine Remanenz der elektrisch leitfähigen Phase aufweist. Diese Abhängigkeit vom elektrischen Feld scheint zunächst in Analogie zu den vorherigen Kapiteln beschriebenen elektrischen Feld induzierten Übergängen zu stehen. Das dies nicht notwendigerweise der Fall ist, macht die Auftragung in Abb. $3.27 \mathrm{~d}$ des Widerstandes im charakteristischen Feld von 7,5T gegen das elektrische Feld deutlich. In der stufensenkrechten Richtung ist der relativ niedrige Widerstand nicht dramatisch vom elektrischen Feld abhängig, während das Feld in der stufenparallelen Richtung einen Übergang induziert, der aber im wesentlichen reversibel ist, d.h. nach Abschalten des Feldes wird 

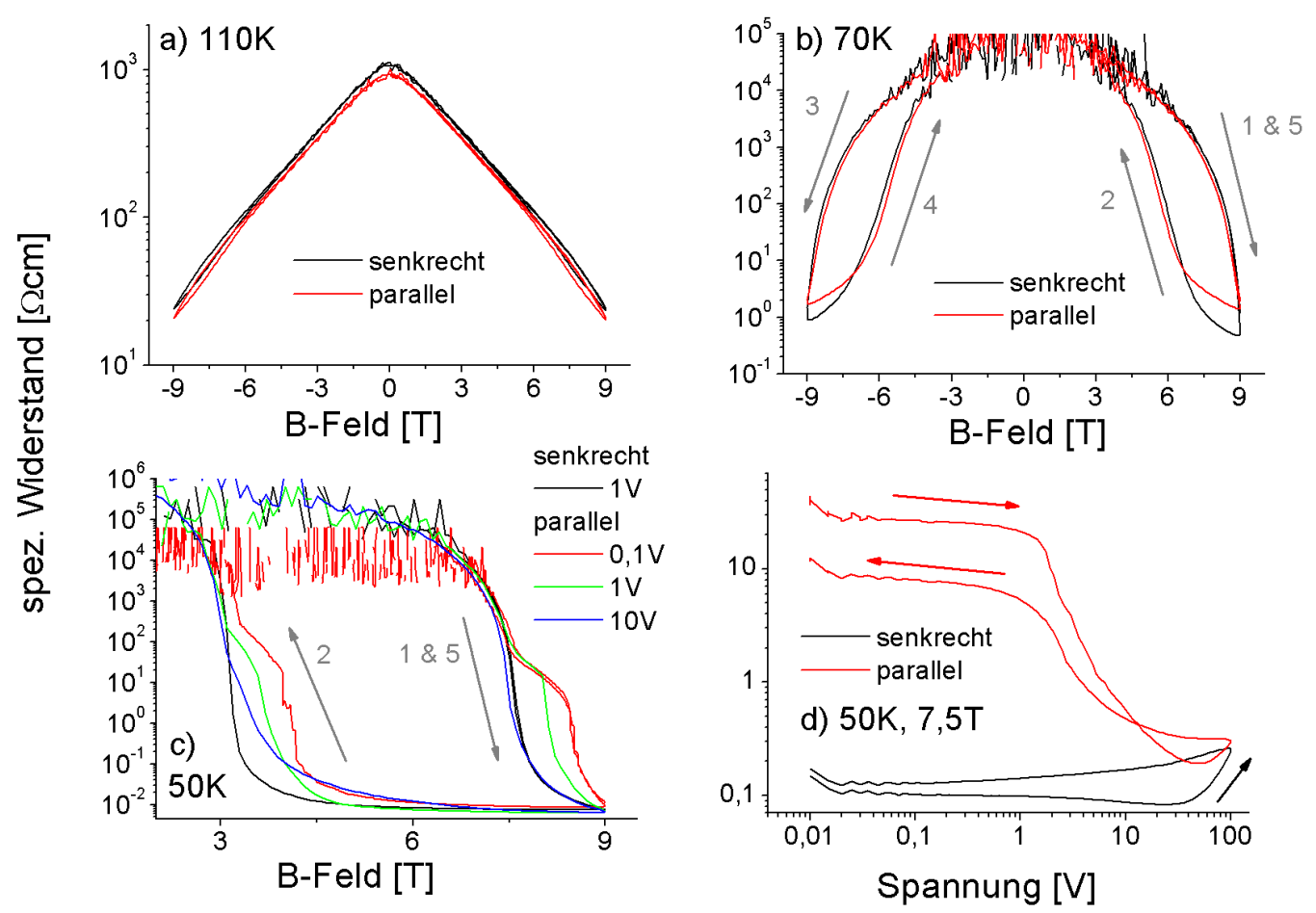

Abbildung 3.27: spezifischer Widerstand über das angelegte Magnetfeld bzw. über die Spannung, im Vergleich Messrichtung senkrecht und parallel zu Substratstufen; Kurven für $110 \mathrm{~K} \mathrm{a}), 70 \mathrm{~K} \mathrm{~b}), 50 \mathrm{~K}$, zusätzlich mit Variation der angelegten Spannung c); Kurve bei $50 \mathrm{~K}$ und $7,5 \mathrm{~T}$ über die Spannung d); Probe Viz- $3_{3 h}$.

wieder ein sehr hoher Widerstand gemessen. Zwar ist nach Durchlaufen der elektrischen Schleife der Widerstand deutlich abgesenkt, aber weniger dramatisch als man nach dem feldinduzierten Übergang auf planaren Substraten erwarten würde. Hierbei muss aber berücksichtigt werden, dass dieser Effekt bei Temperaturen (bei dieser speziellen Probe) auftreten, bei denen keine Remanenz der elektrisch leitfähigen Phase vorliegt. Untersuchungen an unstrukturierten Filmen auf vizinalen Substraten haben gezeigt, dass die Anisotropie im Widerstandsverhalten mit zunehmenden Magnetfeld systematisch unterdrückt wird, für die Anisotropie im Endwiderstand für tiefe Temperaturen siehe Einsatz Abb. 3.28. Des Weiteren kommt es auch durch Langzeitauslagerungen bei hohen Temperaturen zur Unterdrückung der Anisotropie (Abb. 3.28, Probe nach $20 \mathrm{~h} @ 950^{\circ} \mathrm{C}$ ). Dies hat weitreichende Folgen für eine mögliche Optimierung einer ausgeprägten „CMRAnisotropie“. 


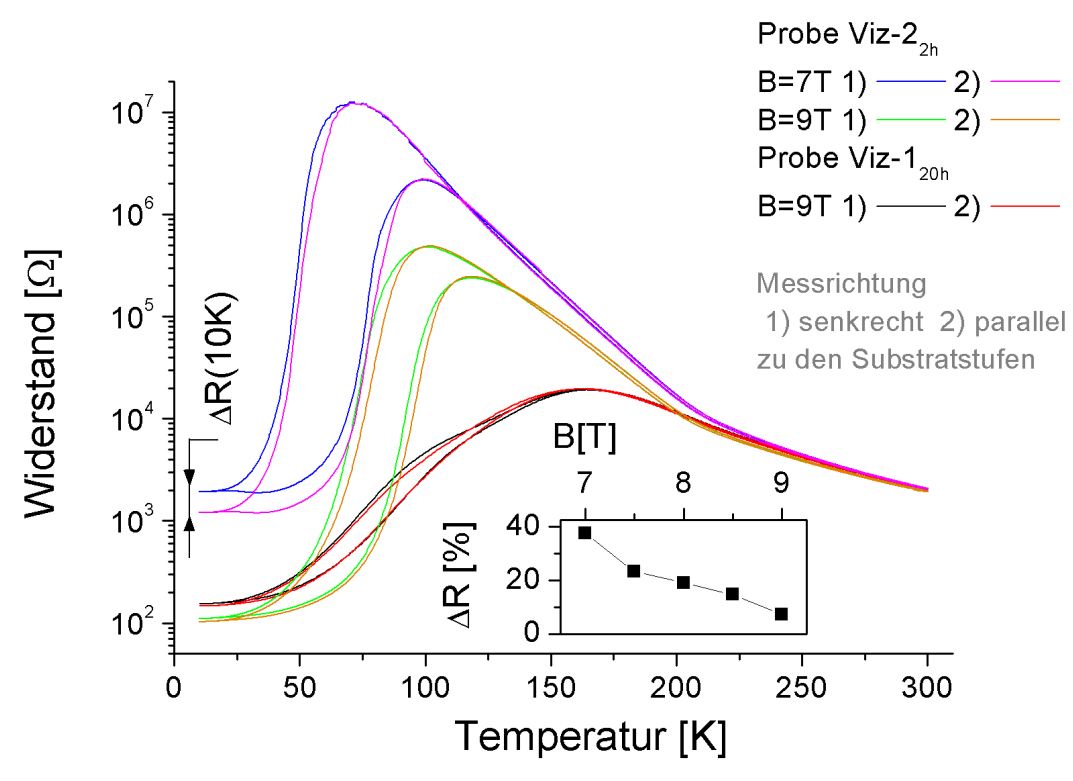

Abbildung 3.28: Transportmessung Widerstand über die Temperatur für die Proben Viz- $1_{20 h}$ und Viz- $2_{2 h}$ auf vizinalen Substraten abgeschieden (mit gleichen Herstellungsparametern), aber unterschiedlichen nachträglichen thermischen Behandlungen; Einsatz: aus den Endwiderständen berechnete Anisotropie über das Magnetfeld für die Probe Viz- $22 h$.

Durch die nachträgliche Auslagerung wird der CMR in der Hinsicht verbessert, dass sich die Übergangstemperatur für den Metall-Isolator-Übergang zu höheren Temperaturen verschiebt. Auf der anderen Seite können die gewollt eingebrachten periodischen Defekte der vizinalen Substrate ausheilen, somit verschwindet die Richtungsanisotropie im elektrischen Transportverhalten. 


\section{Kapitel 4}

\section{Diskussion}

Um im Folgenden die Leitfähigkeitsmechanismen zu diskutieren, muss man vom Standpunkt der Struktur aus drei Phasen unterscheiden. Oberhalb der Ordnungstemperatur liegt vorwiegend die orthorombische Phase Pbnm vor. Unterhalb der Ordnungstemperatur kann diese in die ladungs- und orbitalgeordnete Phase $P 2_{1} n m$ übergehen, wobei dieser Übergang jedoch durch Defekte unterdrückt werden kann. Diese Phase ist unterhalb von ca. 160K antiferromagnetisch. In einem hinreichend starken Magnetfeld vollzieht sich bei tiefen Temperaturen ein weiterer Übergang in die ferromagnetisch- metallartige Phase ${ }^{1}$, wobei dieser Übergang ohne die geordnete Phase nicht möglich ist [66].

Eine Skizze der Tieftemperaturphase $P 2{ }_{1} m n$ ist in Abb. 4.1 dargestellt. Das ausgezeichnete Strukturelement dieser Phase ist eine Mn-O-Mn Bindung, in der ein zudotiertes Loch lokalisiert ist, das Zener-Polaron. Die Tieftemperaturphase lässt sich als eine ladungsgeordnete Phase auffassen, in der die lokalisierte Ladung ein Kompositpolaron darstellt [9, 102]. Das Zener-Polaron weist in der Struktur den flachsten Bindungswinkel auf, der sich von allen anderen Bindungswinkeln unterscheidet. Dies stellt den Gitteraspekt des Polarons dar. Die magnetischen Momente der beteiligten Mangan-Ionen sind dabei im Sinne eines Doppelaustausches parallel ausgerichtet, was den magnetischen Aspekt des Kompositpolarons darstellt. Bei tiefen Temperaturen, bei denen praktisch nur die geordnete Phase vorliegt, weisen die Zener-Polaronen eine antiferromagnetische Ordnung des CETyps auf. In einem hinreichend starken Magnetfeld kann die Zener-Polaronen geordnete Tieftemperaturphase in die ferromagnetisch-metallartige Phase überführt werden. Hierbei müssen die Momente der Zener-Polaronen parallel ausgerichtet werden. Dies hat zur Folge, dass, im Sinne eines Doppelaustausches, ein Ladungstransfer zwischen Zener-Polaronen begünstigt wird. Die enge Korrelation zwischen der Struktur und den Eigenschaften von PCMO zeigt Abb. 4.2. In der antiferromagnetischen Phase ist die Probe isolierend. Mit dem Übergang in die

\footnotetext{
${ }^{1}$ auch Metall-Isolator Übergang, kurz MIT genannt
} 


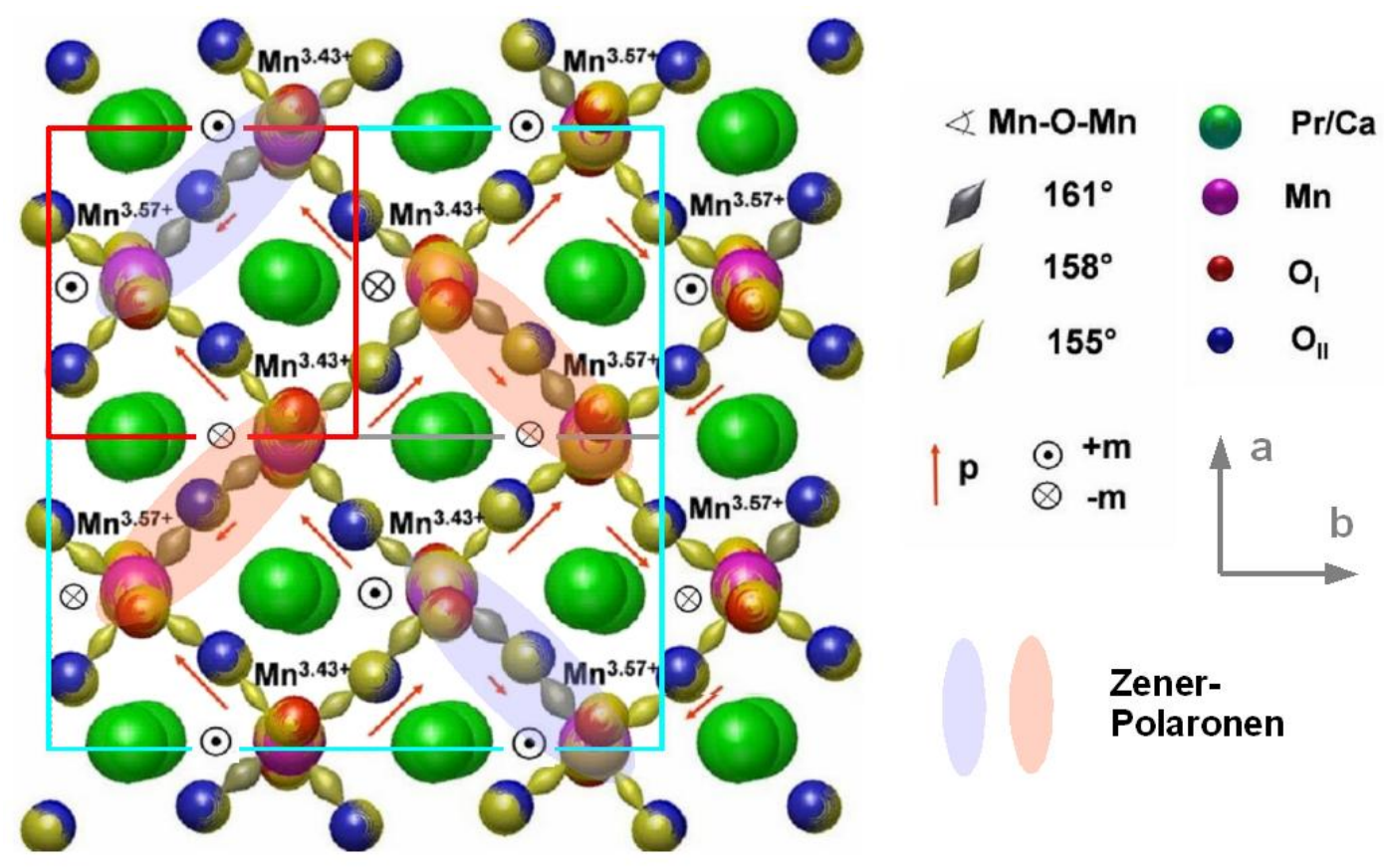

Abbildung 4.1: Strukturprojektion entlang der (001) Richtung für PCMO in der ZenerPolaronen geordneten Phase - P2 ${ }_{1} n m$ Struktur mit eingezeichneten Bindungswinkeln und Manganvalenzen, Verschiebung der Atome gegenüber der ungeordneten Phase in gelb, Ausrichtung der Spins sowie der Polarisation, genaue Beschreibung siehe [42].

ferromagnetisch-metallartige Phase wird die Probe leitfähig. Einen Hinweis auf ein solches Verhalten geben die Neutronenstreuungs-Untersuchungen von Yoshizawa zur Struktur der Tieftemperaturphase im Magnetfeld [97, 96]. Mit dem Übergang werden die Intensitäten der Überstrukturreflexe, die die geordnete Phase anzeigen, schwächer. Sie verschwinden aber nicht. Dies ist ein Anzeichen dafür, dass die ferromagnetisch-metallartige Phase sich strukturell von der ungeordneten Phase Pbnm unterscheidet. Eine mögliche Interpretation dieser Abschwächung der Überstrukturreflexe ist, dass die für die geordnete Phase charakteristischen Unterschiede in den Bindungswinkeln kleiner werden. Da die Magnetisierungskurven beim Abschalten des Feldes praktisch mit der antiferromagnetischen Kurve zusammenfallen (siehe Abb. 4.2 links; praktisch keine Remanenz) kann man zwar nicht notwendigerweise auf einen remanenten (metastabilen) ferromagnetischen Zustand schließen, die hohe Leitfähigkeit und die unveränderten Überstrukturreflexe legen das aber nahe. Die verschwindende magnetische Remanenz könnte auf eine ferromagnetische Domänenstruktur mit leicht beweglichen Domänenwänden zurückzuführen sein. Das bisherige Bild legt die folgende Vermutungen nahe: 

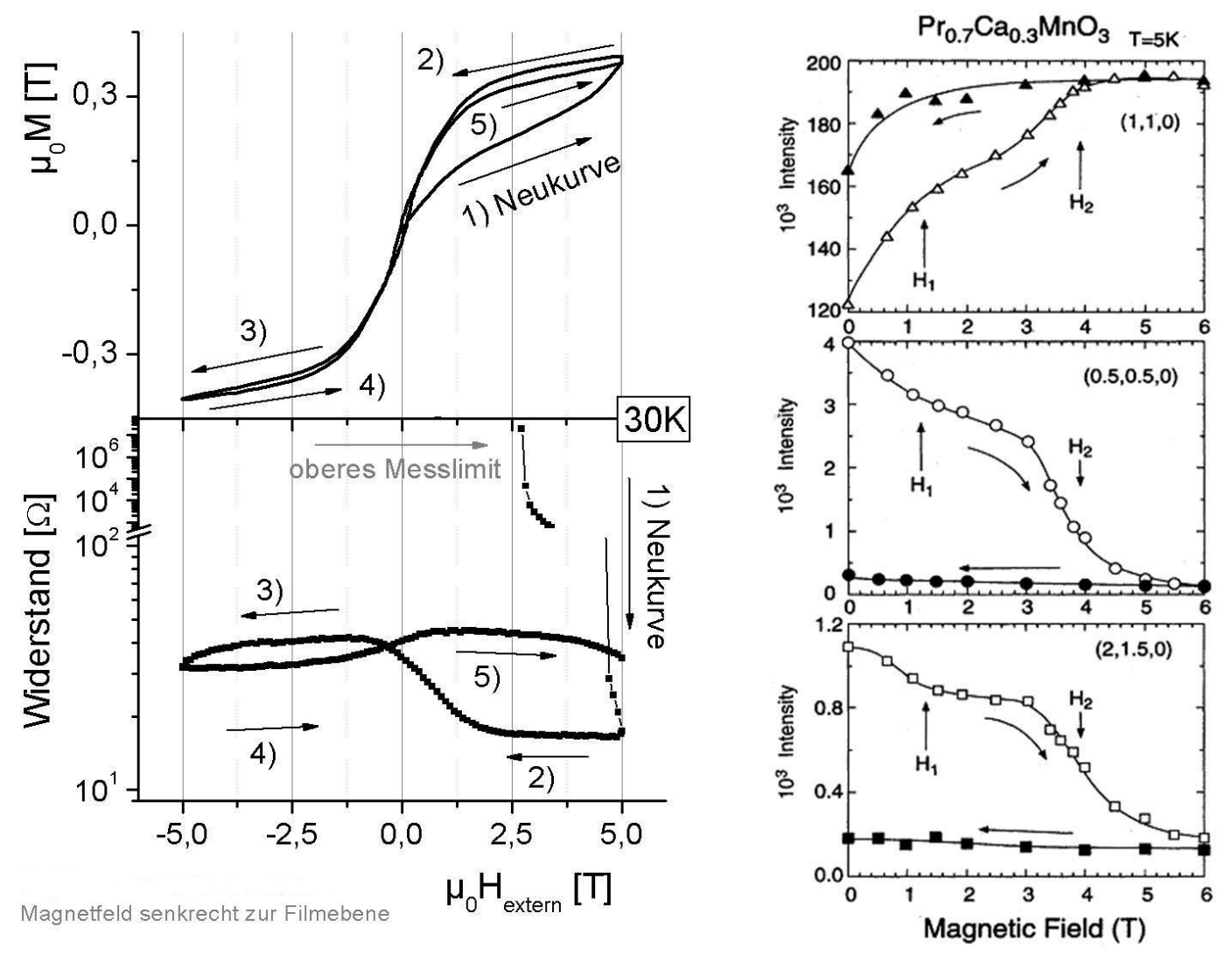

Abbildung 4.2: Vergleich R(B) und M(B) Kurven der Probe PLD- $1_{80 h}$ bei $30 \mathrm{~K}$; rechts Neutronenstreuungs-Untersuchungen [97] - strukturelles Verhalten im Magnetfeld im remanenten Bereich; oben - Reflex für ferromagnetischen Anteil; Mitte antiferromagnetischer Anteil, unten Überstrukturreflex.

Auch in der ungeordneten Phase sind die mobilen Ladungsträger Kompositpolaronen vom Zener-Typ. Eine ferromagnetische Nahordnung im System der beweglichen Polaronen (im ungeordneten Zustand) bedeutet eine Delokalisierung der Ladung und somit geringere Aktivierungsenergien für den Transportprozess. Eine antiferromagnetische Anordnung der Zener-Polaronen hingegen impliziert eine Stabilisierung des kleinen Polarons gegenüber einer Delokalisierung und bedeutet damit höhere Aktivierungsenergien. Im Folgenden soll dieses Szenario auf das Transportverhalten in den unterschiedlichen Temperaturbereichen angewendet werden. 
KAPITEL 4. DISKUSSION

\subsection{Transport in den unterschiedlichen Phasen}

\subsubsection{Elektrischer Transport in der ungeordneten Phase am Beispiel der Aktivierungsenergien}

Bestimmt man aus den Magnetisierungskurven (Abb. 3.9) die Suszeptibilität, so lassen sich die Aktivierungsenergien aus Abb. 3.13 b gegen die paramagnetische Magnetisierung auftragen, Abb. 4.3. In dieser Auftragung fallen die für

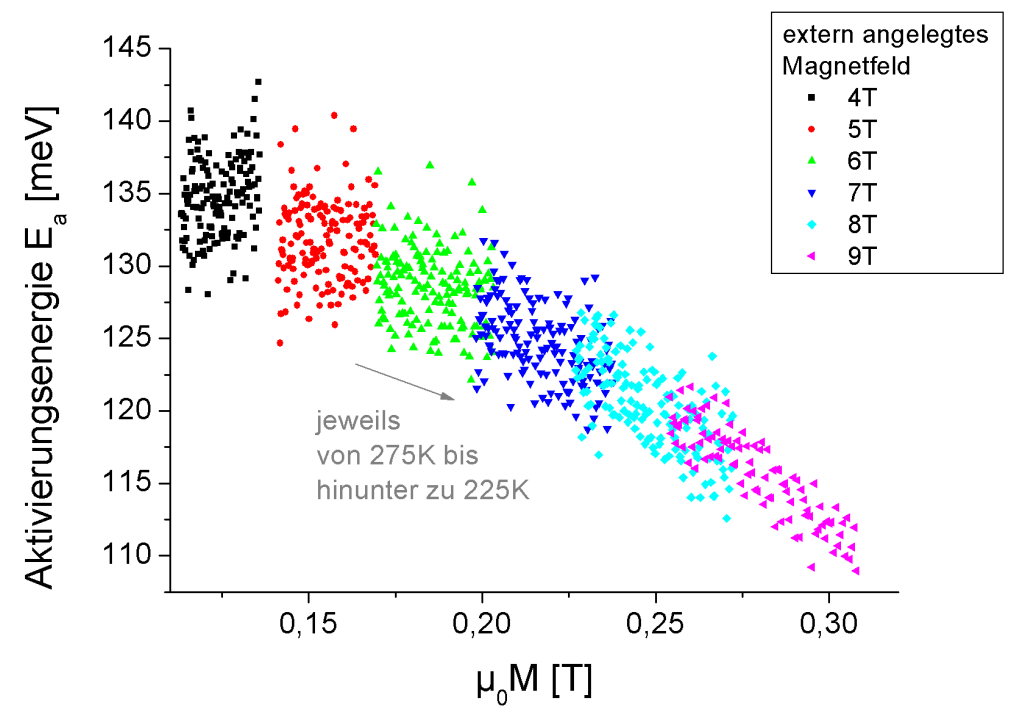

Abbildung 4.3: Aktivierungsenergie über die Magnetisierung, bestimmt aus dem Temperaturbereich $275 \mathrm{~K}$ bis $225 \mathrm{~K}$ für unterschiedliche extern angelegte Magnetfelder.

unterschiedliche Magnetfelder und Temperaturen ermittelten Aktivierungsenergien auf eine Masterkurve. Ähnlich wie bei LCMO [39] ist die Aktivierungsenergie eine Funktion der Magnetisierung. Die Parallelstellung der Momente führt zu einer Absenkung der Aktivierungsenergien, was die eingangs gestellte Vermutung stützt.

\subsubsection{Transport in der ferromagn.-metallartigen Phase}

Bei tiefen Temperaturen liegt bei einem hinreichend starken Magnetfeld die ferromagnetisch-metallartige Phase vor. Die Arbeitshypothese ist, wie zu Beginn von Kapitel 4 dargestellt, dass sich diese, bedingt durch das Magnetfeld, durch eine Strukturänderung aus der Zener-Polaronen (ZP) geordneten Phase bildet. Die 
ZP-geordnete Phase weist eine antiferromagnetische Ordnung des CE-Typs auf. Betrachtet man die Magnetisierungsmessung der nahezu komplett c-Achsen ori-

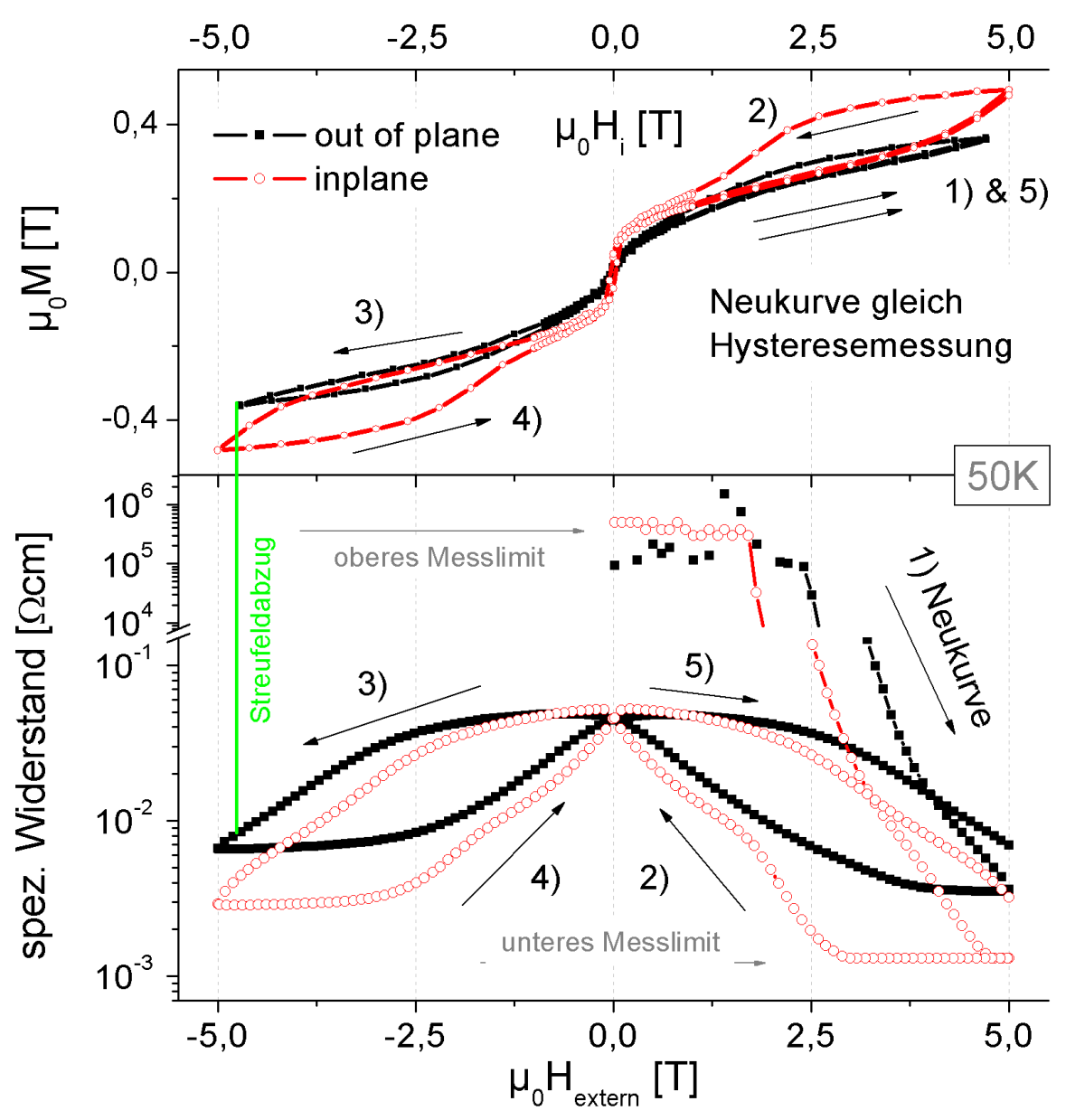

Abbildung 4.4: Vergleich $\mathrm{R}\left(\mu_{0} H_{\text {extern }}\right)$ und $\mathrm{M}\left(\mu_{0} H_{i}\right)$ Kurven bei $50 \mathrm{~K}$, in Hinblick auf Richtungsanisotropie sowohl im magnetischen sowie auch im elektrischen Verhalten; die Nummern 1-5 beschreiben die Reihenfolge der Messungen; Probe PLD- $1_{80 h}$.

entierten Probe PLD-1 $1_{80 h}$ in Abb. 4.4 für den Übergang in die ferromagnetischmetallartige Phase, so ist dieser Übergang für ein Feld in der Ebene viel ausgeprägter. Es ist leichter die Spins um $90^{\circ}$ aus ihrer Position - die sie in der ZP geordneten Phase besitzen - heraus zu drehen, als einen Spin-Flip zu induzieren. Auch ist es schon für kleinere Magnetfelder möglich, einige Spins entlang des angelegten Magnetfeldes auszurichten, was sich in einem früheren Anstieg der Magnetisierung widerspiegelt. Dabei sind in Abb. 4.4 die Magnetisierungsmessungen gegen das innere Magnetfeld $H_{i}$ aufgetragen, der Streufeldbeitrag wurde vom 
externen Magnetfeld $H_{\text {extern }}$ abgezogen, wobei dieser kleiner als der beobachtete Unterschied ist. Diese Richtungsabhängigkeit ist auch in den Transportmessungen wieder zu finden. Zum Einen vollzieht sich der CMR-Effekt für ein in der Filmebene angelegtes Feld bei niedrigeren Magnetfeldern, zum Anderen sind die erreichten Widerstände kleiner. Schaltet man das magnetische Feld wieder ab, so verbleibt für beide Magnetfeldrichtungen fast keine magnetische Remanenz (Feld in der Ebene $40 \mathrm{mT}$, senkrecht $15 \mathrm{mT}$ ). Die elektrische Remanenz hingegen ist für beide Magnetfeldrichtungen im Nullfeld gleich, was so nicht zu erwarten gewesen wäre. Dies bedeutet, dass das Magnetfeld - wie bereits beschrieben - eine Strukturänderung hervorruft, die den CMR-Effekt zur Folge hat. Es liegt daher nahe, dass die Strukturänderungen in der Tieftemperaturphase die Existenz eines metastabilen Zustandes erlaubt, der gegen kleine thermische Fluktuationen stabil ist und so die Aufrechterhaltung einer ferromagnetischen Ordnung und einer hohen Leitfähigkeit ermöglicht. Die sehr geringe Remanenz impliziert dann allerdings, dass sich eine magnetische Domänenstruktur mit praktisch verschwindender Gesamtmagnetisierung ausbilden kann.

\subsubsection{Transport im Übergangsbereich $50 \mathrm{~K}<\mathrm{T}<250 \mathrm{~K}$}

Wurden in den beiden vorherigen Fällen die beiden Extrembereiche ungeordnete Phase sowie ferromagnetisch-metallartige Phase erläutert, so untersucht dieser Abschnitt den Übergangsbereich. Dieser ist durch folgende Faktoren gekennzeichnet: Zum Einen nimmt der Volumenanteil der geordneten Phase bei Temperaturerniedrigung deutlich zu (siehe [93, 101], sowie Abb. 3.7). Dabei tritt beim Unterschreiten der Néel-Temperatur $(\approx 150 \mathrm{~K})$ eine antiferromagnetische Fernordnung auf. Betrachtet man in diesem Temperaturbereich die Magnetisierungskurven (Abb. 4.5 a), so sind keine ausgeprägten Übergänge zu sehen. Allerdings finden sich lokale Maxima und Minima in der Steigung $-\frac{\delta M}{\delta B}$ sowohl im Bereich der magnetischen Ordnungstemperaturen, wie auch im Bereich der Ordnungstemperaturen für die Ladungs- und Orbitalordnung. Die Interpretation dieser Details ist aber auf Grund der vorliegenden Mehrphasigkeit nicht einfach. Die isothermen Messungen der Magnetisierung über das Magnetfeld (Abb. 3.9) zeigen für sinkende Temperaturen im Wesentlichen den Übergang vom Paramagnetismus zum Antiferromagnetismus. In diesem Temperaturbereich finden aber wesentliche Änderungen in der Leitfähigkeit statt (Abb. 4.5 c,d). Somit ist der Übergangsbereich nicht durch eine einfache Korrelation von Magnetisierung und Widerstand gekennzeichnet, sondern geprägt durch die Phasenseparation zwischen Polaronen-geordneter und Polaronen-ungeordneter Phase.

In diesem Zusammenhang ist das Ordnungsmaximum in der Aktivierungsenergie (Abb. 3.13) bei Temperaturen von ca. $190 \mathrm{~K}$ von besonderem Interesse. Er wird mit dem Einsetzen der Ladungsordnung identifiziert [57]. Allerdings würde man dann erwarten, dass ein überwiegender Volumenanteil der Probe geordnet 


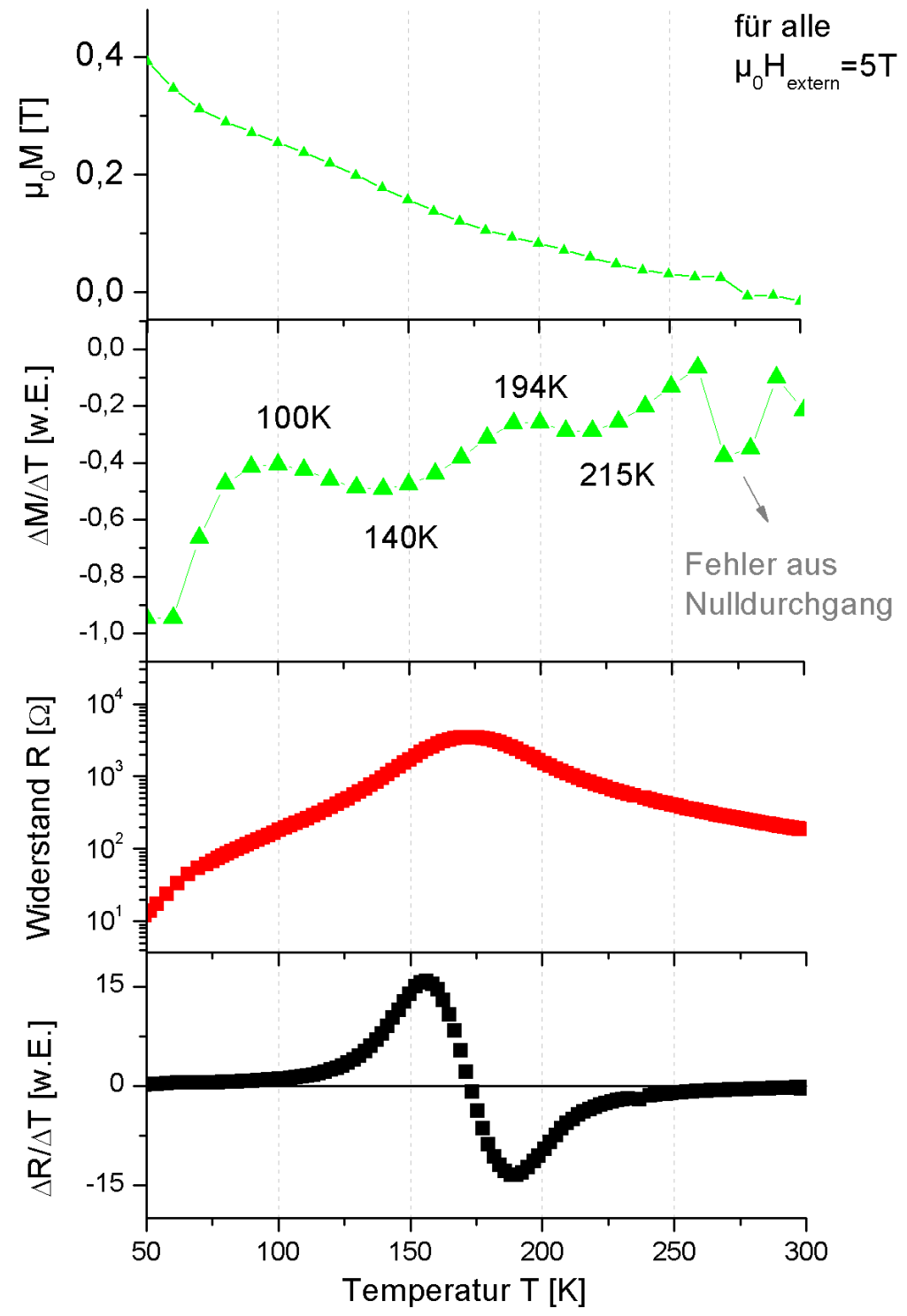

Abbildung 4.5: Über die Temperatur aufgetragen die Magnetisierung sowie der Widerstand jeweils mit der Ableitung nach der Temperatur; Probe PLD-1 ${ }_{80 h}$.

wäre, was nach den vorliegenden Daten [42] aber eher unwahrscheinlich ist. TEM Untersuchungen an 20h ausgelagerten PLD Filmen zeigten, dass bei $\mathrm{T} \approx 190 \mathrm{~K}$ der Anteil geordneter Phase eher kleiner 50\% ist, im Unterschied zu Volumenproben, bei denen der Ordnungsgrad bei $190 \mathrm{~K}$ eher über $75 \%$ erreicht. Wahrscheinlicher ist, dass eine antiferromagnetische Nahordnung in der ungeordneten Phase einsetzt, welche für das Ordnungsmaximum in der Aktivierungsenergie 
verantwortlich ist. Ein glasartiges Verhalten für den Ordnungsübergang mit einer einfrierenden Spindynamik werden zum Beispiel für LCMO diskutiert [77]. Das Auftreten eher langsamer Zeitskalen ist insofern bedeutsam, da nun auch strukturelle Änderungen möglich erscheinen. Die Kombination aus einer strukturellen und einer magnetischen Nahordnung sollte eine deutliche Änderung der Aktivierungsenergie hervorrufen. Im Bild der Zener-Polaronen bricht ein Magnetfeld die Lokalisierung der Zener-Polaronen und damit die antiferromagnetische Nahordnung. Damit kann zwanglos die Unterdrückung des Ordnungsmaximums im Magnetfeld erklärt werden.

\subsection{Die Rolle der Defekte}

Bislang wurden die Eigenschaften an der nahezu defektfreien Probe PLD- $1_{80 h}$ diskutiert. Um nun die Rolle von Defekten näher zu untersuchen, bieten sich besonders solche Proben an, die einen CMR-Effekt erst als Folge nachträglicher Auslagerungen zeigen.

\subsubsection{Nachträgliche thermische Behandlung}

\section{Interdiffusion}

Durch die nachträgliche thermische Behandlung der Filme wurde die Defektstruktur der Filme signifikant verändert. Dies änderte auch gravierend die elektrischen Transporteigenschaften. Jedoch konnte durch die Auslagerung eine Interdiffusion zwischen Substrat und Film auftreten (vergleiche Abschnitt 3.1.5). Betrachtet man das Phasendiagramm für $\operatorname{Pr}_{1-x}\left(\mathrm{Ca}_{1-y} \mathrm{Sr}_{y}\right)_{x} \mathrm{MnO}_{3}$ ([84], Abb. 4.6 a), so ändern sich für eine Strontium-Dotierung bis $\mathrm{y}=0,3^{2}$ nur die Néelsowie die Ordnungstemperatur $(\mathrm{CO} / \mathrm{OO})$. Oberhalb einer Strontium-Dotierung von $y=0,3$ kommt es zum Übergang zu einer ferromagnetisch-metallartigen Phase, was natürlich auch die elektrischen Transporteigenschaften dramatisch beeinflusst (siehe Abb. 4.6 rechts). Es kommt bereits im Nullfeld zu einem Metall-IsolatorÜbergang. Ein solches Verhalten wurde für die langzeit ausgelagerten Proben nicht beobachtet. Auch die hohen Übergangstemperaturen der Probe PLD-1 $1_{80 h}$ lassen sich nicht auf einen Dotierungseffekt zurückführen, da die Probe PLD- $2_{80 h}$ trotz identischer Auslagerung nicht annähernd hohen Übergangstemperaturen zeigt. Nach den SIMS-Messungen im Konzentrationsprofil über die Substrat-Film Grenzfläche (Abb. 3.10) wären auch keine dramatischen Effekte zu erwarten. Zusammenfassend muss der Einfluss der Auslagerung primär vom Standpunkt des Ausheilens von herstellungsbedingten Defekten und nicht als Folge einer zusätzlichen Dotierung diskutiert werden.

\footnotetext{
${ }^{2} \mathrm{Im}$ Folgenden ausgehend von einer Kalzium-Dotierung von $\mathrm{x}=0,35$
} 
a)

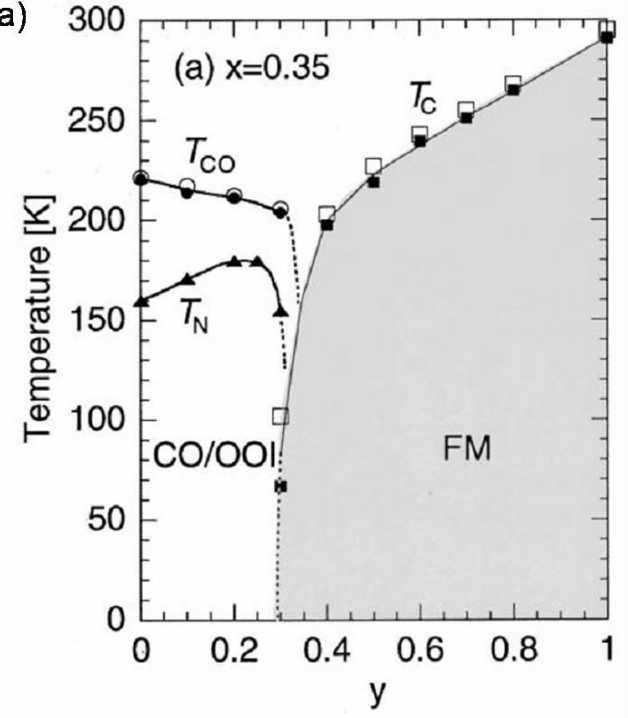

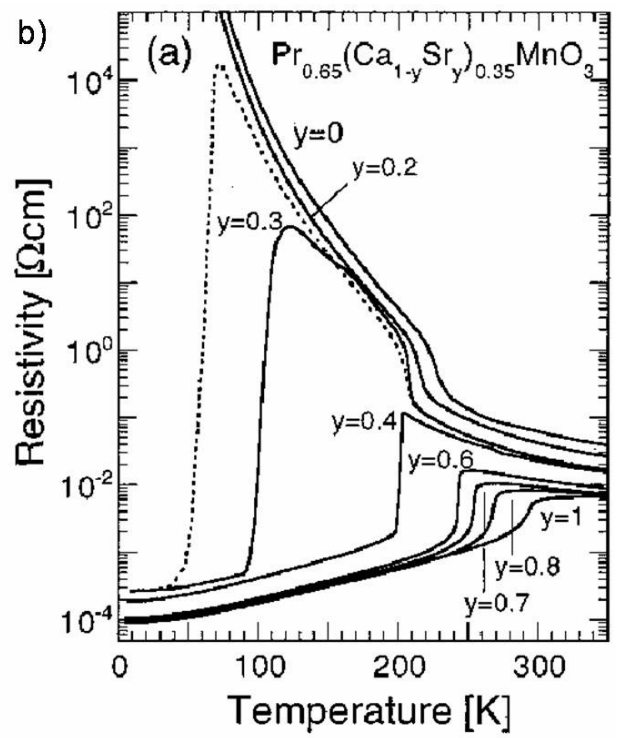

Abbildung 4.6: a) Phasendiagramm PCSMO über Sr-Dotierung; b) R(T)-Kurven für verschiedene Sr-Dotierungen [84].

\section{Spannungen und herstellungsbedingte Defekte}

Die nachträgliche thermische Behandlung hat zunächst einen großen Einfluss auf die mittels Röntgendiffraktometrie ermittelten Dehnungen im Film. Für die Proben PLD-1 und PLD-2 ist die Änderung der Reflexlagen über die Auslagerungszeit in Abbildung 4.7 aufgetragen. In Abbildung 4.8 sind die auf EinkristallGitterkonstanten bezogenen Dehnungen eingetragen. Ausgehend von einem reinen Fehlpassungsmodell würde man für PCMO auf STO in der Filmebene eine Zugspannung von $1,4 \%$ erwarten $^{3}$. Bei prinzipiell gleichen Herstellungsparametern lassen sich Filme mit Dehnungen in einem Bereich von $-0,15 \%<\epsilon_{004}<0,6 \%$ im wie hergestellten Zustand bzw. nach Kurzzeitauslagerung herstellen. Durch die nachträgliche Auslagerung treten zum Teil deutliche Änderungen in den Dehnungen auf, jedoch ohne dass eine klare Systematik erkennbar ist. Vergleicht man diese Ergebnisse mit denen für ein reines Fehlpassungsmodell, so lassen sich diese nicht mit dem Modell erklären. Auch ein einfaches defektorientiertes Modell, indem zum Beispiel intrinsische Spannungen [90] als Folge depositionsbedingter Defekte auftreten, scheint nicht geeignet zu sein. Es ist schwer zu verstehen, warum kleine subtile Veränderungen in den Depositionsbedingungen das Vorzeichen der - nicht gerade kleinen - Dehnungen verändern können. Durch das Ausheilen der Defekte infolge der Langzeitauslagerung bei sehr hohen Temperaturen würde man

\footnotetext{
${ }^{3}$ ausgehend von c-Achsen orientieten Wachstum
} 


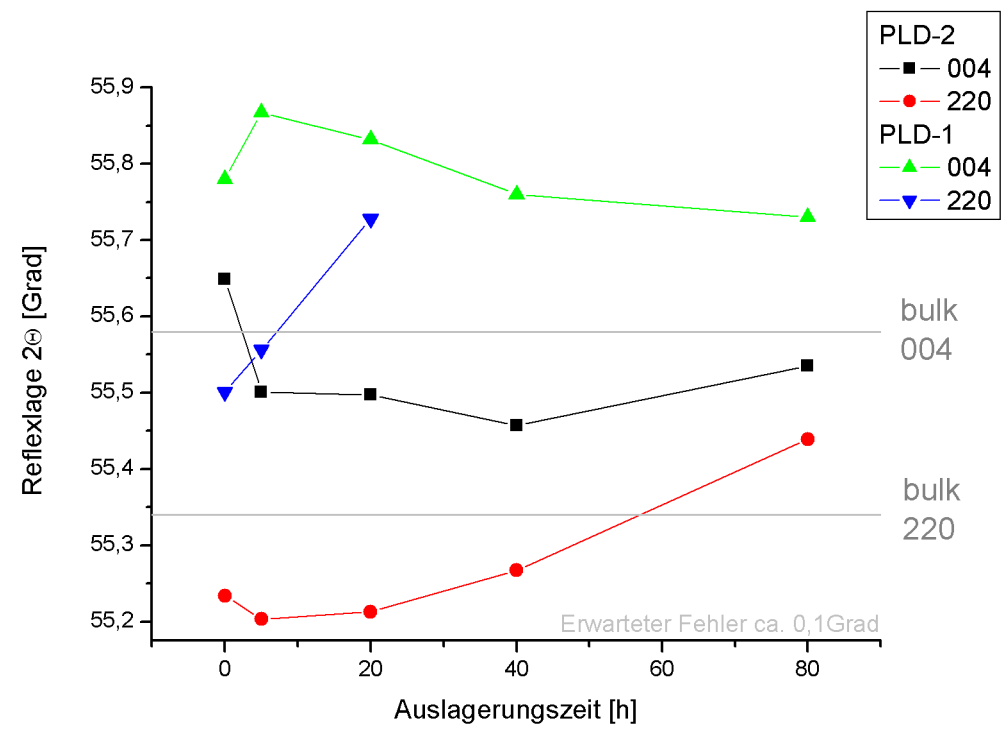

Abbildung 4.7: Entwicklung der (220) und (004) Reflexe über die Auslagerungszeit für die Proben PLD-1 und PLD-2.

erwarten, dass die Unterschiede zwischen den verschiedenen Filmen sich deutlich verringern. Dies wird jedoch nicht beobachtet. Betrachtet man nun weiter die Metall-Isolator-Übergangstemperaturen, so konnte ebenfalls keine einfache Korrelation zu den Dehnungen festgestellt werden (siehe Abb. 4.8). Es können sowohl zug- wie auch druckverspannte Filme einen CMR-Übergang aufweisen. An Hand der zwei eingezeichneten Auslagerungsserien ist vielmehr zu erkennen, dass sich die Übergangstemperatur systematisch zu höheren Temperaturen verschiebt, wohingegen für die Dehnungen keine Systematik zu erkennen ist. Wie im Folgenden aber deutlich werden wird, weisen auch die elektrischen Transportmessungen auf eine Form von Unordnung hin, die am ehesten als lokale Störungen im oktaedrischen Verkippsystem gedeutet werden können. Das aber mit der Auslagerung signifikante Änderungen in der Mikrostruktur verbunden sind, haben Untersuchungen mittels Transmissionselektronenmikroskopie (TEM) in Abschnitt 3.1.3 gezeigt.

Die untersuchten Filme wiesen unterschiedliche Zwillingskonfigurationen auf, die sich sowohl in ihrer räumlichen Ausdehnung (Abb. 3.6), als auch in der Dichte unterschieden. Nanozwillinge traten dabei nur in wie hergestellten Filmen auf. 


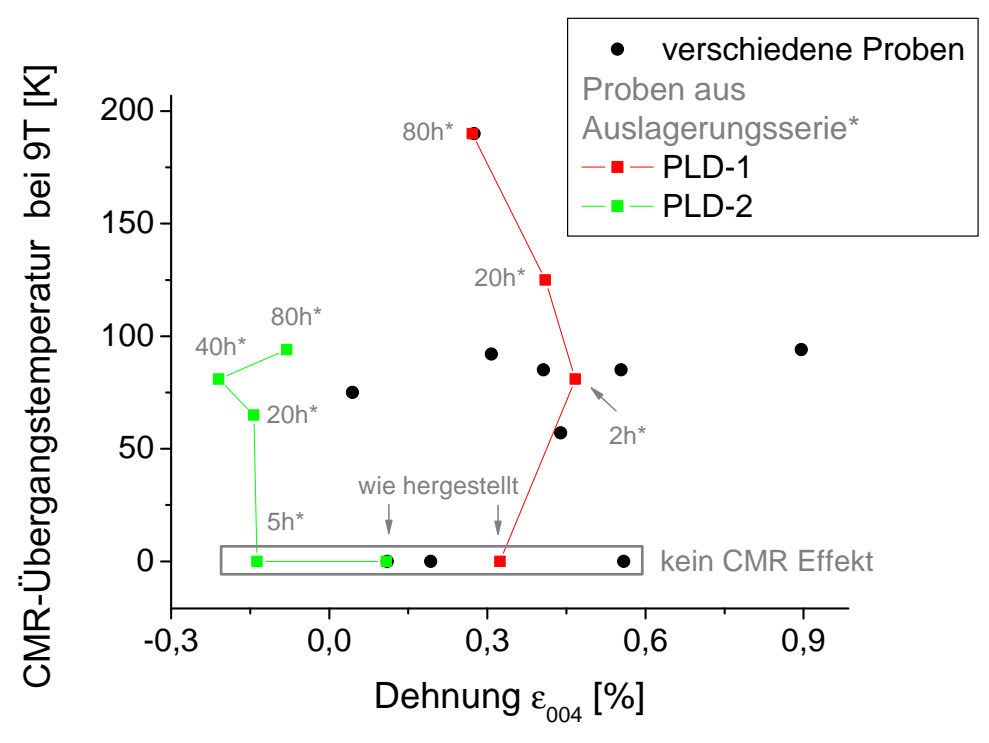

Abbildung 4.8: Übergangstemperatur zu Dehnung für verschiedene Proben sowie für die Proben aus der Auslagerungsserie.

Durch eine thermische Nachbehandlung kam es zum Wachstum des c-Achsen orientierten Domänen auf Kosten solcher mit der (110)-Fehlorientierung. Betrachtet man die Probe PLD-1 aus der Auslagerungsserie in Abb. 3.5, so ist im wie hergestellten Zustand eine ausgeprägte Verzwilligung zu sehen. Nach einer Auslagerungszeit von $80 \mathrm{~h}$ bei $900{ }^{\circ} \mathrm{C}$ an Luft liegt der Film in einer reinen c-Achsen Orientierung vor, siehe auch Abb. 3.6. Dem gegenüber zeigt die Probe PLD- $2_{80 h}$ nach gleicher Auslagerung noch immer eine Verzwillingung.

Die starke Verzwillingung in den Manganaten wird häufig als Konsequenz des strukturellen Phasenübergangs von der kubischen Hochtemperaturphase in die orthorombische Tieftemperatur-Phase gedeutet [6, 35, 48]. Im relevanten Dotierungsbereich liegen für PCMO für diesen Phasenübergang keine Übergangstemperaturdaten vor. Nach den Daten für die anderen Manganate können die Temperaturen aber im Bereich der typischen Depositionstemperaturen für die Filmherstellung bzw. für die typischen Auslagerungstemperaturen liegen. Dies kann eine Ursache für die deutlichen Abhängigkeit der ermittelten Dehnungen von den exakten Depositionsbedingungen sein.

Da die Größe der Zwillingsdomänen aber im Bereich einiger $10 \mathrm{~nm}$ liegt, erscheint es zweifelhaft, dass die Verzwillingung alleine die Ursache für die deutlichen Unterschiede in den MI-Übergangstemperaturen ist. Letztendlich muss man sich die Defekte wohl eher als eine Form der Unordnung im Kippsystem der (im We- 
sentlichen als starr angenommenen) Sauerstoff-Oktaeder vorstellen. Defekte, wie die Zwillingsgrenzen und die Sauerstoffleerstellen, aber auch Zwangsbedingungen, wie starre Kopplung des Films an das Substrat, können Störungen induzieren, die in dem verknüpften Verkippsystem nicht mehr einen lokalen, sondern eher einen langreichweitigen Charakter haben. Hinweise darauf, dass Spannungsfelder auf einer mesoskopischen Längenskala wesentlich sind, werden im Kapitel 4.4 in den vizinalen Substraten aufgeführt.

\subsubsection{Einfluss von Defekten auf die Aktivierungsenergie}

Das am Anfang dieses Kapitels diskutierte thermisch-aktivierte Polaronen (TAP)Modell beschreibt die Polaronenbeweglichkeit als Diffusion in einem streng periodischen Potential, vernachlässigt also die Defekte. Der andere Grenzfall ist das "variable-range-hopping" (VRH)-Modell, dass einen Transport in einem statistischen - durch die Defekte modulierten - Potential entspricht [5, 11]. Nach [88, 79] sowie eigenen Anpassungen stellt das TAP-Modell die bessere Näherung für unsere Schichten dar. Defekte sollten im Sinne von „Trappingcentern" eher zu einer

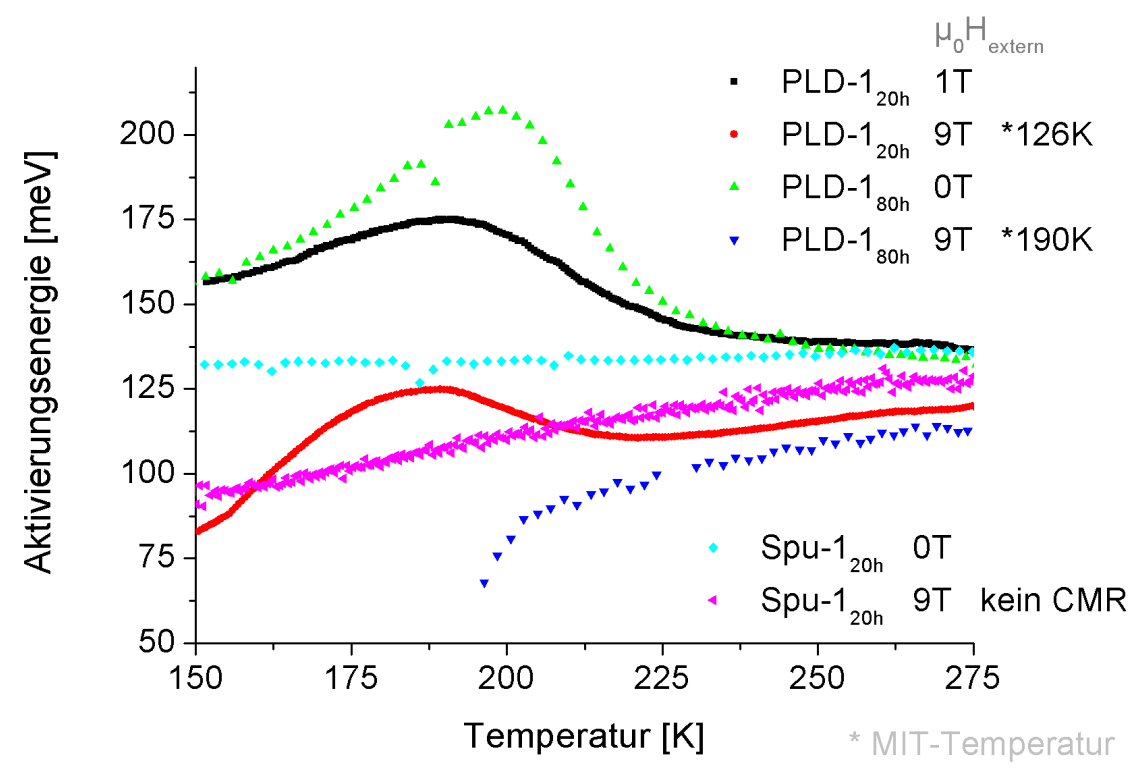

Abbildung 4.9: Aktivierungsenergie über Temperatur.

Erhöhung der Aktivierungsenergie führen [5]. Für eine solche Fragestellung ist es besser die temperaturabhängige differentielle Aktivierungsenergie zu betrachten (Abb. 4.9). Die Aktivierungsenergie für hohe Temperaturen nimmt bei Ausla- 
gerung für die Probe PLD-1 nun wie erwartet im Nullfeld $a b^{4}$. Auch wird an diesem Bild klar, dass, wenn man eine Mittelung über die Temperatur durch eine Arrhenius-Auftragung durchführt, man fälschlicherweise den Eindruck erhält, dass die Aktivierungsenergie mit der Auslagerung zunimmt.

Betrachtet man die Defekte als Verankerungszentren für die Zener-Polaronen, so ist die Abhängigkeit der Aktivierungsenergie über die Auslagerungszeit, also dem Ausheilen von Defekten, verständlich. Defekte unterdrücken die Ausbildung der geordneten Phase, behindern daher sicherlich auch die Nahordnung, was bedeutet, dass die Aktivierungsenergie über die Temperatur sich nicht oder nur wenig ändert.

Inzwischen kann man einige Regeln für den Zusammenhang zwischen der Aktivierungsenergie bei höheren Temperaturen und der MI-Übergangstemperatur aufstellen. Wie der Begriff „Regeln“ hier andeutet, gibt es sehr wohl Abweichungen von diesem Verhalten.

1. Proben, welche keinen CMR aufweisen, zeigen kein oder ein nur sehr schwach ausgeprägtes Ordnungsmaximum.

2. Die unter eins genannten Proben weisen im Wesentlichen eine lineare Abnahme der Aktivierungsenergie mit der Temperatur auf, wobei die Änderung im Magnetfeld nur sehr klein sind.

Proben mit ausgeprägten CMR zeigen oberhalb des Ordnungsmaximums eine temperaturunabhängige differentielle Aktivierungsenergie und deutliche Feldabhängigkeiten.

3. Durch die Auslagerung sinkt die benötigte Aktivierungsenergie im Bereich der Raumtemperatur.

4. Proben, die auch nach Langzeitauslagerungen keinen CMR zeigen, weil sie zum Beispiel bei zu niedrigen Temperaturen hergestellt wurden, zeigen nur eine leichte Absenkung der Aktivierungsenergie durch die Auslagerung, wobei sich die Steigung $\frac{d E_{a}}{d T}$ kaum ändert.

Betrachtet man nun den mittleren Temperaturbereich im Hinblick auf die Magnetfeldabhängigkeit, so unterscheiden sich die Magnetisierungskurven (Abb. 3.9) für deutlich unterschiedliche Metall-Isolator-Übergangstemperaturen nicht dramatisch. Dies ändert sich, wenn man die tiefen Temperaturen betrachtet. Hier finden sich besonders kleine Widerstände nur in Proben, deren Magnetisierungskurven einen Übergang zum Ferromagnetismus anzeigen. Die enge Korrelation von Struktur, Magnetismus und Leitfähigkeit kommt hier wieder zum Ausdruck. Defekte verschieben den metamagnetischen Übergang zu tieferen Temperaturen

\footnotetext{
${ }^{4}$ Niedrige Magnetfelder haben noch keinen Einfluss auf die Aktivierungsenergie (siehe z. B. Abb. 3.15), daher kann für die Probe PLD- $1_{20 h}$ die 1 T Messung als Nullfeldmessung angenommen werden
} 
(Abb. 3.9). In dem bisher verfolgten Szenario sind die starken Abhängigkeiten des Widerstandes und der Aktivierungsenergie bei hohen und mittleren Temperaturen die Folge der magnetisch vermittelten Wechselwirkungen zwischen den Zener-Polaronen. Hier greifen die Defekte an, sie behindern diese Wechselwirkungen. Bei einer hinreichend hohen Dichte an Defekten unterdrücken diese die Ausbildung einer Ladungsordnung. Damit verbunden bildet sich die geordnete Tieftemperaturphase nicht aus. Ein solches von der Unordnung abhängiges Stabilisierungskriterium für kleine Polaronen hat z. B. D. Emin [21] vorgeschlagen. Allerdings schließt eine solche Unterdrückung der Fernordnung Nahordnungen nicht aus. Das in der Literatur diskutierte „glasartige“ Verhalten des Polaronensystems oberhalb der Ordnungstemperatur [2, 16, 58, 91], spiegelt sich, speziell am Beispiel des CER-Effektes, auch in Proben wieder, die keinen CMR zeigen [79]. Was die Natur der Polaronen bei tiefen Temperaturen in einem starken Magnetfeld angeht, wobei es hier sogar zu einer Strukturveränderung kommt, wird im letzten Abschnitt dieses Kapitels diskutiert.

\subsection{CMR- und CER-Effekt}

Bislang wurde für die Bestimmung der Transporteigenschaften - sowie der davon abgeleiteten Aktivierungsenergie - davon ausgegangen, dass das elektrische Feld und damit der elektrische Strom nur zu einem ohmschen Verhalten Anlass gibt. Dem ist jedoch nicht so, gerade im Übergangsbereich von der geordneten Phase in die ferromagnetisch-metallartige Phase kann das elektrische Feld eine große Rolle spielen. Für hohe Ströme kommt es über den gesamten untersuchten Temperaturbereich zu Nichtlinearitäten in den Strom-Spannungs-Kennlinien, wobei der Widerstand teilweise um einige Größenordnungen einbrechen kann (Kolossaler Elektrowiderstandseffekt, CER-Effekt). Der CER induziert sogar einen Ordnungs-Unordnungsübergang [42]. Das bisher diskutierte Zener-Polaronen Modell gilt nur für kleine Ströme. Daher muss beachtet werden, in welchem Zustand sich die Probe befindet.

\subsubsection{Zustandsdiagramm}

Aus den Strom-Spannungs-Kennlinien (Abb. 3.19) kann ein Zustandsdiagramm erstellt werden (siehe Abb. 4.10), indem man die charakteristischen Punkte der Spannungs-Strom-Kennlinien (gemessen als Strom) gegen die Temperatur aufträgt. Die Ströme definieren dabei die „cross-over"-Bereiche, in denen sich die lokalen Ableitungen der Kennlinien gravierend verändern. Die Stromdichten bzw. die elektrischen Felder in Kombination mit den angelegten magnetischen Feldern können für die jeweiligen Übergänge stark von der Defektdichte der Probe abhängig sein. Dies ist schon aus den unterschiedlichen Metall-Isolator-Übergangstem- 


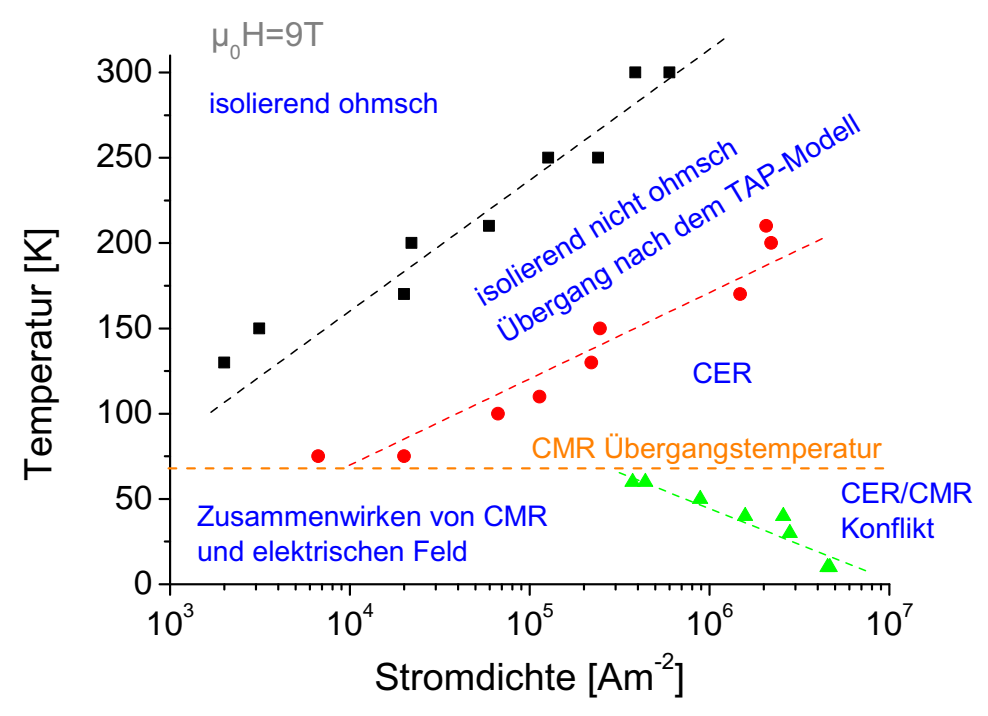

Abbildung 4.10: Zustandsdiagramm aus den UI-Kurven in Abb. 3.19, Probe Spu- $2_{a p}$.

peraturen für die verschiedenen Defektdichten ersichtlich. In diesem Sinne sollte man das Zustandsdiagramm als probenspezifisch auffassen.

Folgende Regeln gelten aber für alle Proben:

1. Bei kleineren Temperaturen werden niedrigere Stromdichten benötigt, um den ohmschen Bereich und damit den Gültigkeitsbereich für das TAPModell zu verlassen.

2. Auch die charakteristischen Stromdichten für den Übergang in den Zustand des CER-Effektes werden mit sinkender Temperatur kleiner.

3. Unterhalb der Metall-Isolator-Übergangstemperatur gibt es einen Bereich, in dem das Magnetfeld und das elektrische Feld zusammen die Leitfähigkeit erhöhen (Abb. 3.20). Aber auch unterhalb der Übergangstemperatur kommt es für hohe Stromdichten zum Übergang in den Zustand des CEREffektes. Dabei zeigt sich ein entgegengesetztes Verhalten. Je niedriger die Temperatur ist, umso höher sind die benötigten Stromdichten. Es besteht dort eine Konkurrenz zwischen CER und CMR-Effekt. 


\subsubsection{CMR- und CER-Effekt als konkurrierende Effekte}

Wie aus dem Zustandsdiagramm zu erkennen ist, tritt der CER-Effekt bereits bei wesentlich höheren Temperaturen als der CMR-Effekt auf. Ein zentraler Unterschied zwischen dem CER- und dem CMR-Effekt ist, dass der CER-Effekt keine geordnete Phase erfordert. Befindet sich die Probe im Zustand des CER-Effektes, so ist der Widerstand nahezu magnetfeldunabhängig (siehe Abb. 4.11). Befindet

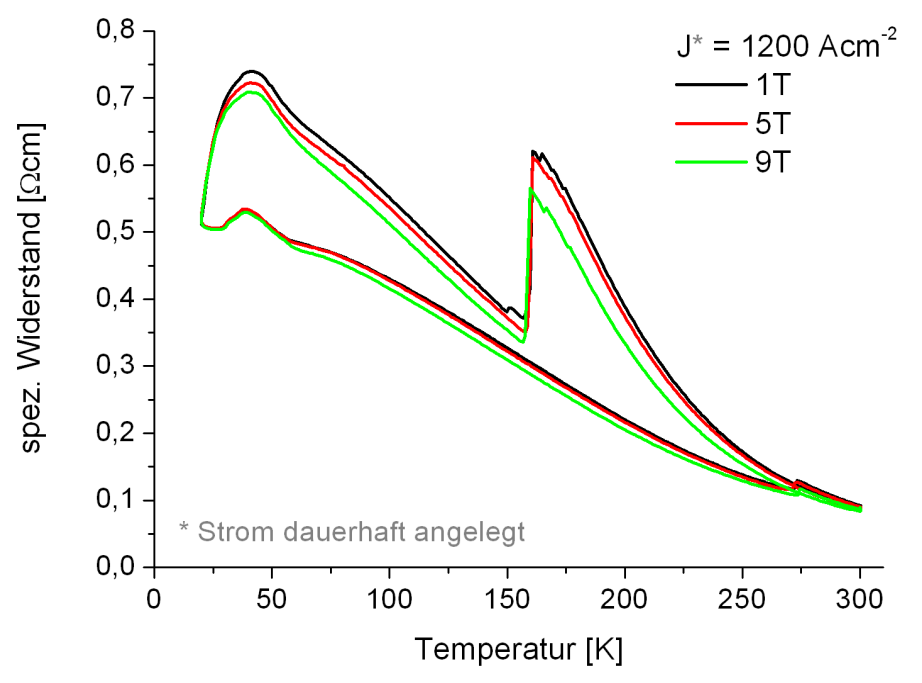

Abbildung 4.11: Widerstandsverlauf im CER-Effekt; das Magnetfeld hat annähernd keinen Einfluss; Probe Spu- $2_{a p}$.

sich die Probe hingegen im Zustand des CMR-Effektes, so führt ein hinreichend starker Strom zum CER-Effekt und damit zu einem Ordnungs-Unordnungsübergang. Je nach Defektdichte der Probe kann dabei der Widerstand im Magnetfeld kleiner oder auch größer als im CER-Zustand sein. An Hand der Abb. 3.12 ist die Entwicklung des erreichten Endwiderstandes bei tiefen Temperaturen gut zu erkennen, dieser nimmt trotz gleichbleibenden Magnetfeld mit zunehmender Ausheilung der Defekte ab. Ein solches Verhalten ist für den CER nicht gefunden worden [79]. In kombinierten Feldern ist die Leitfähigkeit magnetfeldunabhängig bzw. nimmt ein Maximum an, je nachdem, ob die alleine im Magnetfeld erreichte Leitfähigkeit kleiner oder größer als im CER-Zustand ist.

Die wesentlichen Konsequenzen aus dieser Betrachtung sind, dass die Abnahme des Widerstandes im Magnetfeld bei tiefen Temperaturen (die Nichtlinearität der Spannungs-Strom-Kennlinie) eher auf Polaronen als auf freie Ladungsträger hindeutet. Dem entgegen verändert sich die Natur der Ladungsträger im 
CER-Effekt. In der elektrisch induzierten leitfähigen Phase koppelt die Leitfähigkeit kaum noch an das Magnetfeld an, es fehlen damit die typischen Aspekte der magnetischen Korrelation zwischen den Zener-Polaronen. Im hochgetriebenen Zustand des CER-Effektes könnten die Ladungsträger weitestgehend vom Gitter entkoppelt sein [21].

Des Weiteren brechen starke elektrische Ströme die Korrelation zwischen den Zener-Polaronen und können so zu einer Widerstandserhöhung führen. Die muss jedoch mit Vorsicht betrachtet werden. Nach [25] bilden sich stromführende Pfade im CER-Zustand aus, dessen Querschnitte unbekannt sind. Der über die Probendimensionen bestimmte scheinbare spezifische Widerstand kann damit deutlich größer sein als der reale spezifische Widerstand der stromtragenden Filamente. Wenn man den Ladungstransport im Zustand des CMR als polaronisch, im Zustand des CER als eher frei auffasst, so scheint sich bei höheren Temperaturen bzw. nicht zu geringen Defektdichten der CER durchzusetzen. Für Proben mit sehr hohen Leitfähigkeiten im Magnetfeld konnten bei tiefen Temperaturen nicht so große elektrische Felder angelegt werden, um einen CER-Effekt zu ermöglichen. Es scheint aber nicht unplausibel, dass der polaronische Transport generell eine Instabilität bezüglich hoher elektrischer Felder aufweist. Dies macht auch das Beispiel der elektrisch leitfähigen Remanenz deutlich.

\subsubsection{Die elektrisch leitfähige Remanenz}

Die elektrisch leitfähige Remanenz ist ein weiteres Beispiel für die enge Korrelation zwischen Struktur und Leitfähigkeit. Betrachtet man das Phasendiagramm in Abb. 2.7, so erstreckt sich der Übergangsbereich für niedrige Temperaturen bis zum Nullfeld. Dies weist darauf hin, dass die Struktur der ferromagnetisch leitfähigen Phase auch im Nullfeld erhalten bleibt, was auch die in Abb. 4.2 zitierten Experimente bestätigen. Der metastabile Zustand der elektrisch leitfähigen Remanenz ist dabei zeitlich gesehen stabil. Durch eine Temperaturerhöhung zerfällt die gute Leitfähigkeit kontinuierlich, wobei auch diese Zwischenzustände metastabil sind (Abb. 3.23). Ein irreversibler Übergang aus einem isolierenden in einen leitfähigen Zustand bzw. der umgekehrte Übergang durch Anlegen des elektrischen Feldes wurde in Abb. 3.20 und Abb. 3.24 gezeigt. In der Abb. 3.24 liegt bei $60 \mathrm{~K}$ noch eine hohe Leitfähigkeit im remanenten Zustand vor. Sie steigt zunächst moderat im elektrischen Feld. Mit weiterer Steigung erfolgt ein Übergang in einen isolierenden Zustand, dessen Leitfähigkeit aber noch höher ist als bei einer reinen Nullfeldkühlung. Noch bei $70 \mathrm{~K}$ ist der Unterschied zwischen der remanenten und der Nullfeldleitfähigkeit deutlich kleiner. Nun können in dem remanenten Zustand durch das elektrische Feld deutliche Erhöhungen in der Leitfähigkeit induziert werden, ohne das ein irreversibler Übergang erfolgt. Diese Änderungen in remanenten Zuständen finden aber bei elektrischen Feldern statt, die zu klein sind, um nach einer Nullfeldkühlung signifikante Änderungen 
in der Leitfähigkeit hervorrufen (vergleiche offene Symbole in Abb. 3.24). Bei tiefen Temperaturen ist der remanente Zustand instabil bezüglich Stimulanzen wie Temperaturerhöhung und elektrischen Feldern, die die Polaronenmobilität erhöhen. Über die Mobilisierung der Polaronen können Strukturänderungen induziert werden. Bei hohen Temperaturen weist der remanente Zustand immer mehr Ähnlichkeiten mit dem CER-Verhalten auf. Ohne Magnetfeld zeigt der CER-Zustand starke Vorgeschichtsabhängigkeiten. Induziert man über das elektrische Feld eine hohe Leitfähigkeit, so verbleibt eine Excess-Leitfähigkeit nach Abschalten des Feldes und deutliche Änderungen der Leitfähigkeit können nun durch viel kleinere elektrische Felder induziert werden [79]. Genau dieses Verhalten zeigt Abb. 3.24. Mit der Erhöhung der Temperatur geht der remanente Zustand in einen Zustand über, der dem ähnelt, den man nach einer Nullfeldkühlung nach dem Induzieren hoher Leitfähigkeiten auch ohne Magnetfeld erreicht.

\subsection{Vizinale Substrate}

Die bisherigen Untersuchungen wurden an Proben mit isotroper Defektstruktur gemacht. Ein Ziel dieser Arbeit stellte die gezielte Beeinflussung der Struktur mittels vizinaler Substrate dar. Für eine anisotrope Defektstruktur würde man Unterschiede in den Transporteigenschaften erwarten, ähnlich wie in Hochtemperatursupraleitern auf vizinalen Substraten [33]. Die auf vizinalen Substraten hergestellten Schichten zeigen ein geändertes Wachstum (vom Insel- zum „stepflow"-Wachstum), siehe auch Abb. 3.3. Betrachtet man die Oberfläche der Filme für hohe Verkippwinkel, so weist die Anordnung der parallel zu den Substratstufen verlaufenden Oberflächenwellen bereits auf die anisotrope Defektdichte hin. Die weiteren Defekte in der Struktur (z. B. herstellungsbedingte Defekte) sind dabei isotrop über die Probe verteilt. Dies kann aus den fast identischen spezifischen Widerständen sowie den nahezu gleichen Aktivierungsenergien in der ungeordneten Phase entnommen werden (Abb. 3.15).

Die für Filme, welche auf vizinalen Substraten abgeschieden wurden, erwarteten periodischen Antiphasengrenzen (siehe Abschnitt 2.2.3), wurden in PCMO nur vereinzelt in großen Abständen gefunden. Damit können Anitphasengrenzen eigentlich keinen dominanten Einfluss auf das Transportverhalten haben.

Die Grenzen in der Verwendung von vizinalen Substraten sollen an dieser Stelle nicht unerwähnt bleiben. Diese liegen in der benötigten nachträglichen Auslagerung zur Verringerung der herstellungsbedingten Defekte. Für einen gut ausgeprägten CMR-Effekt ist diese nachträgliche thermische Behandlung nötig. Sie beseitigt aber nicht nur die herstellungsbedingten Defekte, sondern auch die durch die vizinalen Substrate eingebrachten Defekte. Somit ist eine Optimierung der Richtungsanisotropie auf vizinalen Filmen durch eine nachträgliche Auslagerung nur in Grenzen möglich. 
Ein deutlicher Unterschied in den Schichten auf vizinalen Substraten gegenüber denen auf planaren Substraten ist in den Dehnungen zu finden. Eine erste Serie

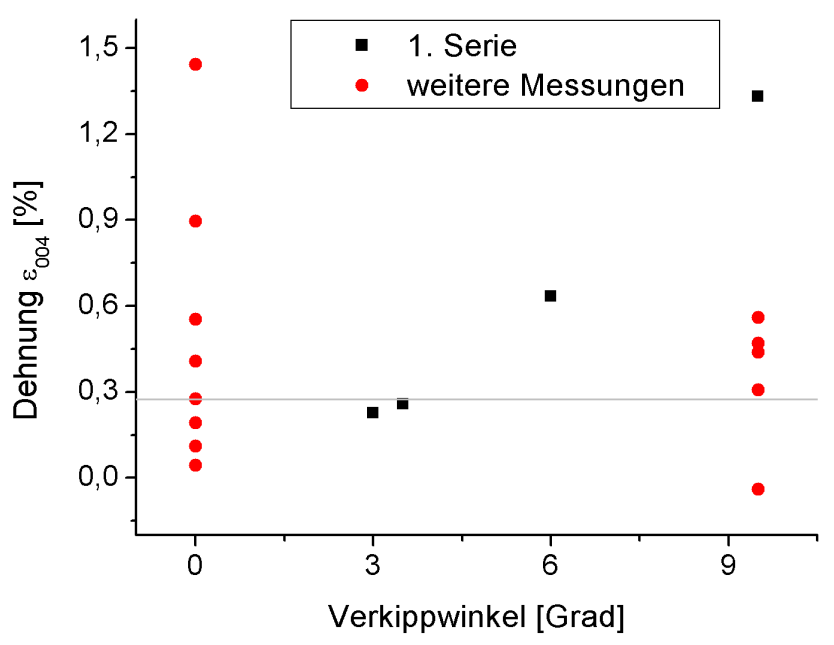

Abbildung 4.12: Dehnung in der Substratebene über den Verkippwinkel für eine erste Serie (Proben hintereinander hergestellt), sowie für weitere später hergestellte Proben.

zeigte eine Abhängigkeit der Dehnungen im Film vom Verkippwinkel der Substrate. Diese konnte jedoch für später hergestellte Filme nicht bestätigt werden (Abb. 4.12), wobei man das oben beschriebene komplizierte Wachstum bedenken muss. Für Filme mit Inselwachstum wird eine anisotrope Verspannung theoretisch nicht erwartet, im Gegensatz dazu in Filmen mit step-flow Wachstum schon. Dabei ist zunächst zu berücksichtigen, dass in Abb. 4.12 die Dehnung infolge der Querkontraktion bestimmt wurde. Um Aussagen über die Anisotropie der Spannungen in der Filmebene zu treffen, muss man Reflexe vermessen, die bezüglich ihrer kristallographischen Richtung eine Komponente parallel zum Substrat aufweisen. Röntgendiffraktogramme auf einen Flächendetektor zeigten für Filme auf planaren Substraten keine Anisotropie in der Dehnung. Filme auf vizinalen Substraten wiesen hingegen anisotrope Dehnungen auf. In Abbildung 4.13 links ist ein Röntgendiffraktogramm auf einen Flächendetektor dargestellt. Der mit 3 gekennzeichnete Reflex ist der (110)-STO Reflex. Über diesen Reflex wurde die Skala geeicht, wodurch eine Bestimmung der Netzebenenabstände möglich wurde. Die Reflexe 1 und 2 gehören zur Schar der (111)-PCMO Reflexe und 1 wird mit (111) sowie 2 mit (11̄1) indiziert. Der Winkel zwischen den beiden Reflexen beträgt auf dem Flächendetektor $90^{\circ}$, was bedeutet, dass sie Gitterebenen angehören, die senkrecht aufeinander stehen. Reflex 1 hat mit einem Netzebenenabstand von 3,49 eine Dehnung von ca. 1,2\%, Reflex 2 mit 3,52 Netzebenenabstand eine Deh- 


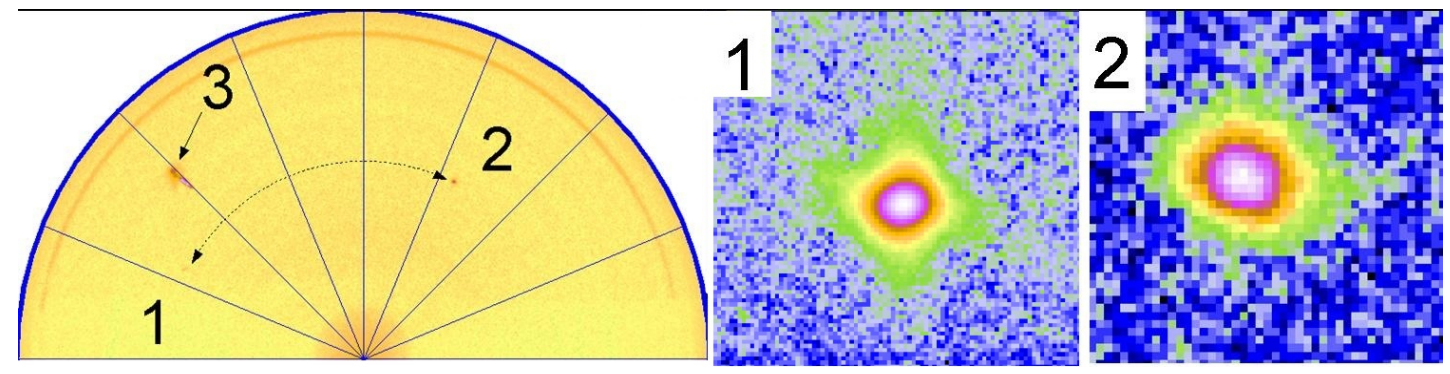

Abbildung 4.13: links: Röntgendiffraktogramm auf Flächendetektor, Gitter zur Orientierung, rechts: Reflexe 1 und 2 vergrößert; Probe Viz-3 $3 h$ (auf vizinalem Substrat).

nung von ca. 2\%. Beide Reflexe sind kreisrund und weisen damit keine zusätzliche Verteilung der Halbwertsbreite in eine Raumrichtung auf. Der Unterschied in den Gitterparametern der (111) und (111) Reflexe lässt auf eine anisotrope Dehnung in der Ebene schließen. Auf Grund der Verzwillingung der Filme können keine Rückschlüsse auf die Richtung der Anisotropie gezogen werden.

Zusammenfassend kann gesagt werden, dass vizinale Substrate eine anisotrope Defektstruktur in Form von anisotropen Spannungen in der Filmebene einbringen. Diese ist aber nicht dramatisch, wie aus den sehr ähnlichen Aktivierungsenergien (Abb. 3.15) sowie spezifischen Widerständen in der ungeordneten Phase zu schließen ist. Betrachtet man die Transporteigenschaften der Filme auf vizinalen Substraten in Hinblick auf eine Anisotropie (Abb. 3.16, Abb. 3.27), so stellt man fest, dass eine Richtungsanisotropie im Transportverhalten im Übergangsbereich zu finden ist. Wie man Abb. 3.14 entnehmen kann, können die Richtungsanisotropien in diesem Bereich beträchtlich werden. Da eine isotrope Verteilung der herstellungsbedingten Defekte sowie wenige Antiphasengrenzen vorliegen, sind die anisotropen Spannungen in der Filmebene sehr wahrscheinlich verantwortlich für die Richtungsanisotropien in den Transporteigenschaften. Dies ist insofern auf planaren Substraten konsistent mit den Ergebnissen, bei denen Filme mit unterschiedlicher Dehnung unterschiedliche MI-Übergangstemperaturen aufweisen, auch wenn es keine systematische Korrelation gab. Die anisotropen Spannungen haben wahrscheinlich Auswirkungen auf die Struktur, z. B. in dem die Verkippungen der $\mathrm{MnO}_{6}$ Oktaeder, und damit die Größe der Mn-O-Mn Bindungswinkel in der Filmebene verändert werden.

Ein enger Zusammenhang der Spannung mit der Übergangstemperatur sowie dem benötigten Magnetfeld wird auch in der Literatur diskutiert [94, 73]. In einem starken elektrischen Feld, wo man eher von mobilen Polaronen ausgehen muss, spielen die Spannungen weniger eine Rolle, so dass die Anisotropie verschwindet (Abb. 3.27). 


\subsection{Ladungsträger in der ferromagnetisch-me- tallartigen Phase}

Nach den Ausführungen in den vorherigen Abschnitten muss man davon ausgehen, dass sich in der ferromagnetisch-metallartigen Phase ein polaronischer Transport vorliegt. Das am Anfang der Diskussion skizzierte Zener-Polaronen Szenario kann in dieser Phase im Prinzip zu einem bandartigen Transport kleiner Polaronen führen, wie es in [100] für LCMO diskutiert wird. Aber auch ein Übergang zu großen Polaronen erscheint möglich.

Dies soll durch das Anpassen der Messdaten an Modelle für kleine Polaronen (Abschnitt 2.3.2) und große Polaronen (Abschnitt 2.3.3) herausgefunden werden (siehe Abb. 4.14 a). Um etwas über die Güte der Anpassungen der Messdaten an
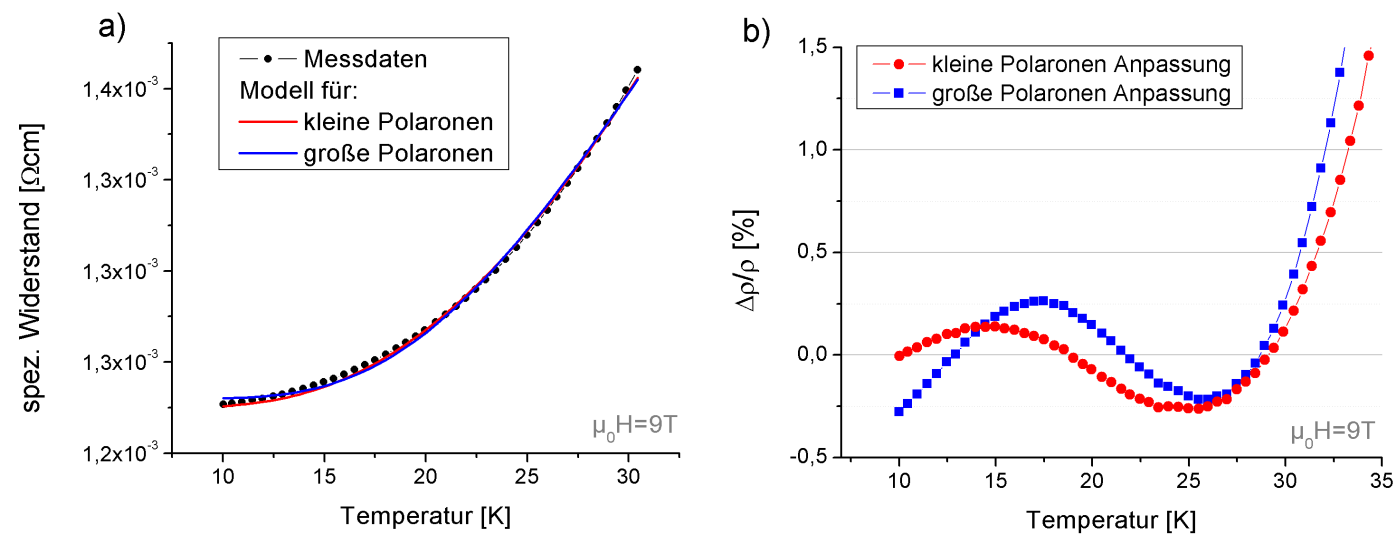

Abbildung 4.14: a: Anpassen von polaronischen Transportmodellen an die Messdaten; b: sich ergebender Fehler der Anpassungen an die Messdaten.

die Modelle aussagen zu können, ist in Abb. 4.14 b der prozentuale Unterschied zwischen den Messdaten und den Anpassungen aufgetragen. Unterhalb von 30 K stellen beide Modelle eine akzeptable Anpassung dar, insbesondere wenn man berücksichtigt, dass andere Streubeiträge wie an Magnonen nicht berücksichtigt sind (vergleiche [100]). Eine klare Tendenz für ein Modell (große bzw. kleine Polaronen) ist ausgehend von der Güte der Anpassungen aber nicht zu finden. Aus beiden Modellen erhält man die Phononenfrequenzen $\omega_{L O}$ (Abb. 4.15), die im Bereich der Mode $\left(1,4 \cdot 10^{13} \mathrm{~Hz}\right)$ liegen, die man im PCMO der Q2 Jahn-TellerMode zuordnet [92]. Auch dies spricht dafür, dass es sich bei den Ladungsträgern für den elektrischen Transport um Polaronen handelt, ohne das man entscheiden kann, welches Modell präferiert wird. Sehr beachtlich ist in diesem Zusammenhang auch die Tatsache, dass für Proben mit höherer Defektdichte - also größerem 


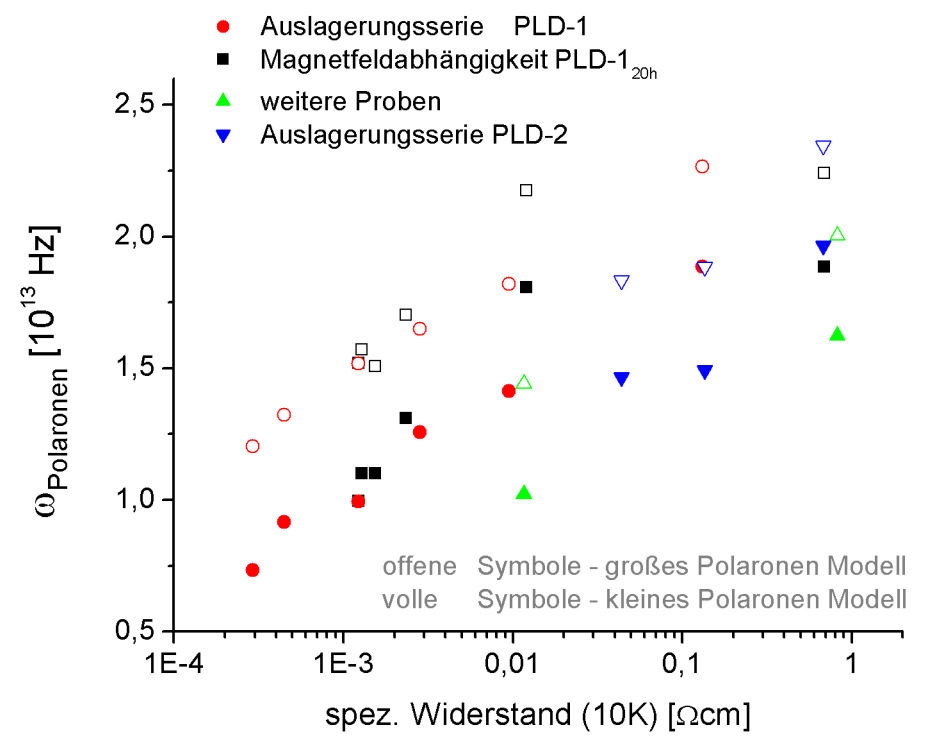

Abbildung 4.15: Aus den Modellanpassungen bestimmte Phononenfrequenzen über den spezifischen Widerstand bei sehr tiefen Temperaturen (10 K).

spezifischen Widerstand - höhere Frequenzen aus den Anpassungen hervorgehen. Ein größerer Anteil an geordneter Phase, Grundlage für die ferromagnetischmetallartige Phase, ist in defektärmeren Filmen zu finden. Ebenso ist der Anteil an ferromagnetisch-metallartiger Phase für höhere Magnetfelder größer. Eine stärker ausgebildetete ferromagnetisch-metallartige Phase bedeutet größere Delokalisierung der Ladungsträger, was mit dem Einfrieren der hochfrequenteren Softmoden einhergeht.

Betrachtet man in der Anpassung für kleine Polaronen nach Abschnitt 2.3.2 den Vorfaktor $C_{M}$, so ist dieser nach [24] proportional zur effektiven Masse des Polarons. Nimmt man den Widerstand bei $10 \mathrm{~K}$ als Maß für den Restwiderstand, so sollte diese Größe im Sinne der relevanten Streuprozesse ebenfalls proportional zur effektiven Polaronenmasse sein. Trägt man $C_{M}$ gegen den spezifischen Widerstand bei $10 \mathrm{~K}$ doppeltlogarithmisch auf, so ergibt sich über viele Größenordnungen ein nahezu linearer Zusammenhang (siehe Abb. 4.16). Dieser lineare Zusammenhang besteht aber nicht nur für unterschiedliche Magnetfelder, sondern auch für unterschiedliche Auslagerungszeiten und damit unterschiedliche Defektdichten. Mehr noch, alle Punkte liegen auf einer Masterkurve, was eine direkte Abhängigkeit des spezifischen Widerstandes bei sehr tiefen Temperaturen mit der effektiven Polaronenmasse widerspiegelt. Im Gegensatz zum vorherigen Modell für kleine Polaronen tritt im Modell nach Feynman [24] (Abschnitt 2.3.3) eine Kopplungskonstante explizit auf. Betrachtet man nach der Anpassung dieses Mo- 


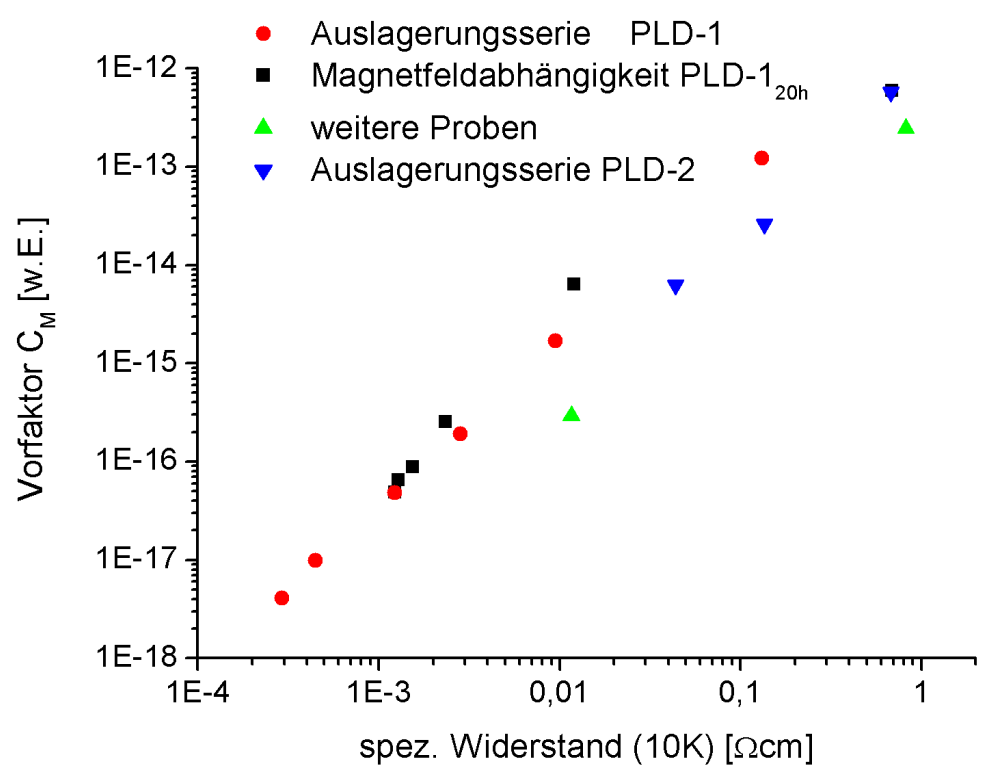

Abbildung 4.16: Aus dem Transportmodell für kleine Polaronen (Formel 2.7) bestimmter Vorfaktor $C_{M}$, der proportional zur effektiven Masse des Polarons ist.

dells den Vorfaktor, welcher von der Kopplungskonstanten $\alpha$ und den davon abgeleiteten Funktionen $v, w$ sowie der Masse der Polaronen abhängt, so ergibt sich ein sehr ähnliches Bild wie für die Anpassung nach dem kleinen Polaronenmodell (siehe Abb. 4.17). Da auch die Kopplungskonstante $\alpha$ in die Masse des Polarons eingeht, kann man sagen, dass für Filme mit größeren spezifischen Widerständen bei sehr tiefen Temperaturen auch die Polaronenmasse größer ist. Als Konsequenz kann man sagen, dass die Ladungsträger in der ferromagnetisch-metallartigen Phase Polaronen sind. Mit steigender Delokalisierung der Ladungsträger, egal ob durch ein größeres Magnetfeld oder defektärmere Filme, kommt es zur Verringerung der Masse der Polaronen. Das Feynman-Modell ist hier in seinem ursprünglichen Sinn (Modellsystem für ein reales Polaron), verwendet worden, in der die Kopplungskonstanten als effektiv vom Magnetfeld und der Dichte abhängige Größen betrachtet wurden. Auch wenn man im Rahmen der verwendeten Modelle das kleine Polaronen-Modell nicht ausschließen kann, so deuten diese Systematiken doch darauf hin, dass beim Übergang in die ferromagnetisch-metallartige Phase sich der Charakter der Ladungsträger von einem kleinen, stark an das Gitter gekoppelten Polaron zu einem großen, schwach an das Gitter gebundenen Polaron ändert und die damit verbundene Delokalisierung des Ladungsträger mit einer Verstärkung des Doppelaustausches und damit eine ferromagnetische Ordnung einhergeht. Dieses Szenario wird für Manganate durch optische Untersuchungen 


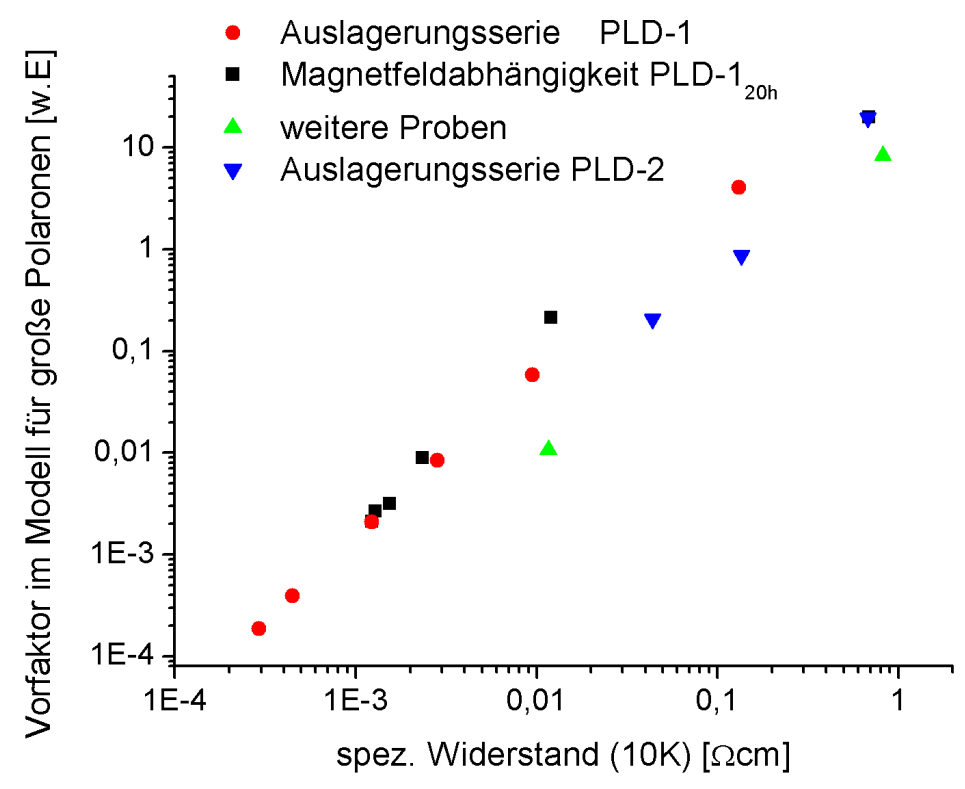

Abbildung 4.17: Aus dem Transportmodell für große Polaronen (Formel 2.8) bestimmter Vorfaktor.

$[34,44,46,54,55,59,60,65,69,70,95]$ gestützt, wobei sich die spektralen Dichten $\mathrm{zu}$ niedriegeren Energien und zu asymmetrischer Form beim Übergang von kleinen zu großen Polaronen verschieben [22, 78]. 


\section{Kapitel 5}

\section{Zusammenfassung}

In der vorliegenden Arbeit wurde das Transportverhalten von PCMO in Abhängigkeit der Defektdichte untersucht. Durch thermische Auslagerungen konnte das Transportverhalten systematisch verändert werden. Dabei wurde festgestellt, dass eine zu hohe Dichte an Defekten zu einer Unterdrückung des CMR-Effektes bzw. zu einer Reduzierung der Übergangstemperatur führt. Dies ist auf die Unterdrückung der geordneten Phase durch die Defekte zurückzuführen, die für die Ausbildung der ferromagnetisch-metallartigen Phase eine notwendige Voraussetzung ist. Ein einfacher Zusammenhang der Übergangstemperatur zur Dehnung konnte in den Filmen auf planaren Substraten nicht festgestellt werden.

Des Weiteren ist es gelungen epitaktische PCMO-Dünnfilme auf vizinalen Substraten abzuscheiden. Dabei ist es zu einem Übergang vom Insel- zum „step-flow“Wachstum gekommen. Es wurde in diesen Filmen eine anisotrope Defektstruktur erzeugt, die sich hauptsächlich als anisotrope Dehnungen in der Filmebene bemerkbar macht. Diese führte zu Richtungsanisotropien in der Leitfähigkeit von Filmen auf vizinalen Substraten.

Die Leitfähigkeit wurde auch durch das Wechselspiel von elektrischem und magnetischem Feld beeinflusst. Hierbei stimulieren sowohl ein elektrisches als auch ein magnetisches Feld einzeln betrachtet den Transport, was zu einer Reduzierung des Widerstandes führt. Dabei werden mit sinkender Temperatur niedrigere Stromdichten benötigt, um sowohl den ohmschen Bereich zu verlassen, wie auch die Probe in den Zustand des CER-Effekt zu überführen. Bei der Kombination der beiden Felder kann das elektrische Feld unterhalb der MI-Übergangstemperatur den Übergang in die leitfähige Phase im Sinne einer erhöhten Polaronenmobilität unterstützen. Wird durch das elektrische Feld ein zu großer elektrischer Strom induziert, so kann es aber auch zum gegenteiligen Effekt kommen, die gute Leitfähigkeit der ferromagnetisch-metallartigen Phase wird zerstört. Dabei sind die benötigten Stromdichten umso höher, je niedriger die Temperatur ist. Befindet sich die Probe im Zustand des CER-Effektes, so hat ein Magnetfeld keinen Einfluss mehr auf das Transportverhalten. Besonders deutlich wurden diese Effekte 
bei tiefen Temperaturen, wo je nach der Vorgeschichte der Abkühlung metastabile isolierende und leitfähige Zustände erreicht werden. Eine Erhöhung der Polaronenmobilität durch elektrische Felder führt hier zu irreversiblen Übergängen in Richtung des durch Magnetfeld und Temperatur festgelegten Gleichgewichtszustandes.

Betrachtet man die Transportmessungen in Hinsicht auf die beteiligten Ladungsträger, so lassen sich die Experimente konsistent in einem Szenario beschreiben, in dem Kompositpolaronen vom Zener-Typ die Ladungsträger sind. Die Aktivierungsenergie verkleinerte sich mit steigender Magnetisierung, wie für magnetische Polaronen erwartet. Die Erhöhung der differentiellen Aktivierungsenergie bei Temperaturen um $190 \mathrm{~K}$ (das „Ordnungsmaximum“) spricht für ein glasartiges Verhalten der magnetischen Ordnung der Polaronen. Diese Fernordnung im Polaronensystem kann durch Defekte unterdrückt werden, wobei eine Nahordnung noch möglich ist. Dabei lassen sich die relevanten Defekte wohl nicht im Bilde einzelner kristallographischer Defekte beschreiben. Vielmehr scheint es sich um eine Unordnung im Kippsystem der Oktaeder zu handeln, die, wie es gerade das Beispiel der vizinalen Substrate deutlich macht, über die resultierenden Spannungen die elektronischen Eigenschaften subtil verändern.

Beim Magnetfeld getriebenen Metall-Isolator-Übergang in die ferromagnetischmetallartige Phase vollzieht sich ein struktureller Übergang. Damit verbunden kommt es in der ferromagnetisch-metallartigen Phase zum scheinbar kontinuierlichen Verkleinern der Polaronenmasse mit steigendem Magnetfeld bzw. mit abnehmendem Grad der Defektdichte. Auch wenn man aus den vorliegenden Transportdaten keine Präferenz für ein kleines- oder großes-Polaronenszenario ableiten kann, beide Modelle erlauben letztendlich akzeptable Anpassungen, so legen es aber die Systematiken nahe, den CMR-Effekt als einen Übergang von kleinen zu großen Polaronen zu beschreiben. 


\section{Anhang A}

\section{Experimentelles}

\section{A.1 Schichtherstellung}

Die untersuchten Dünnfilme wurden mittels gepulster Laserdeposition gefertigt. Das hierfür benötigte Target wurde zuvor in einem Sinterprozess hergestellt. Des Weiteren wird im Folgenden die Substratvorbehandlung sowie die Vorbereitungen des Films für die elektrische Charakterisierung erläutert.

\section{A.1.1 Gepulste Laserdeposition}

Für die Schichtherstellung kam die Herstellungsmethode der gepulsten Laserdeposition (im weiteren kurz PLD) [51, 64, 89] zum Einsatz. Das aus dem Target

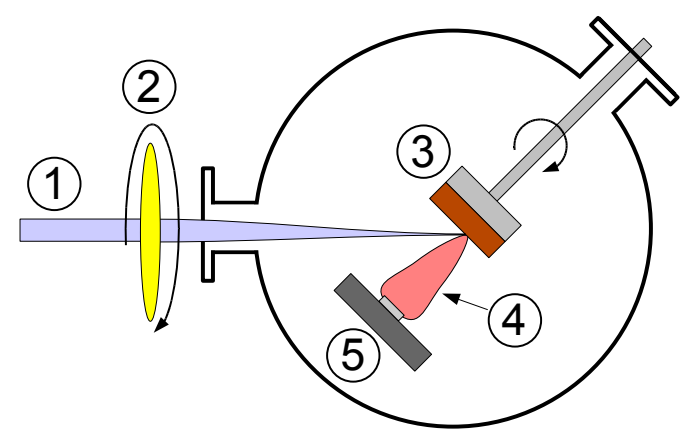

Abbildung A.1: Schemaskizze Eigenbau PLD Anlage.

durch einen Laserpuls ablatierte Material wird auf der Oberfläche des Substrates 
deponiert. Der verwendete Laser ist ein KrF-Excimerlaser von der Firma Lambda Physik des Typs LPX 110i mit einer Wellenlänge von $\lambda=248 \mathrm{~nm}$ bei einer Pulslänge von 30 ns. Die Energiedichte auf dem Target entsprach 1,5 J/ $\mathrm{cm}^{2}$ bei einer Repititionsrate von $6 \mathrm{~Hz}$. Die Herstellungstemperatur betrug $750^{\circ} \mathrm{C}$ bei einem Sauerstoffdruck von 0,2 mbar. Einer der großen Vorteile des PLD ist der Stöchiometrieübertrag vom Target auf das Substrat. Da sich der Laser außerhalb der Depositionskammer befindet, sind zudem relativ hohe Sauerstoffdrücke zur Herstellung möglich.

In Abbildung A.1 ist eine Schemaskizze der verwendeten Kammer abgebildet. Der gepulste Laserstrahl (1) geht durch die asymmetrisch gelagerte rotierende Linse (2), wodurch der fokussierte Laserstrahl das Target (3) abrastert. Dies dient der Erhöhung der Depositionsfläche. Das Target befindet sich auf einem rotierenden Halter, was das gleichmäßige Abtragen fördert, die lokale Überhitzung verringert, sowie zur Vermeidung von Droplets beiträgt. In der Plasmakeule (4) wird das aus dem Target gesprengte Material in stöchiometrischer Zusammensetzung auf das Substrat gebracht. Das Substrat ist auf einem speziell hergestellten Heizer aufgebracht. Dieser wurde für eine homogene Substrattemperatur optimiert, die während der Herstellung mittels Temperatursensoren ständig kontrolliert wurde.

\section{A.1.2 Target}

Das Target wurde aus den Ausgangspulvern $\mathrm{Pr}_{6} \mathrm{O}_{11}, \mathrm{CaCO}_{3}$ und $\mathrm{Mn}_{2} \mathrm{O}_{3}$ hergestellt. Die Ausgangspulver wurden unter Argon-Atmosphäre zuerst ausgeheizt, um mögliche Anlagerungen von Wasser zu beseitigen und anschließend im richtigen stöchiometrischen Verhältnis abgewogen. Danach folgte jeweils dreimal ein Kalzinier- und Mahlprozess. Die Kalzinierung wurde für jeweils 12 Stunden bei $1050{ }^{\circ} \mathrm{C}$ an Luft durchgeführt. Während des Mahlprozesses in einer Kugelmühle wurde das Pulver für jeweils 30 min zermörsert und durchmischt. Im Anschluss wurde aus dem Pulver ein Target gepresst, welches noch einmal bei $1050{ }^{\circ} \mathrm{C}$ für 12 Stunden gesintert wurde. Mittels Röntgendiffraktometrie wurde die Phasenreinheit des PCMO-Targets bestimmt, dass heißt nach diesem Prozess zeigten sich

nur noch die charakteristischen Röntgenreflexe der gewünschten orthorombischen PCMO-Phase.

\section{A.1.3 Substrate}

Als Substrat wurde einkristallines Strontiumtitanat $\mathrm{SrTiO}_{3}($ kurz STO) der Firma Crystek verwendet. STO ist ein einfach kubisches Perowskit mit einer Gitterkonstante von $a=0,3905 \mathrm{~nm}$. Für einige spezielle Experimente wurden diese Substrate in einem definierten Winkel zur (100)-Orientierung schief geschnitten und anschließend poliert. Dieser Winkel $\gamma$ (im Folgenden Verkippwinkel genannt) betrug 3 Grad, 6 Grad bzw 9,5 Grad. Die entstehenden Stufen haben die Höhe 


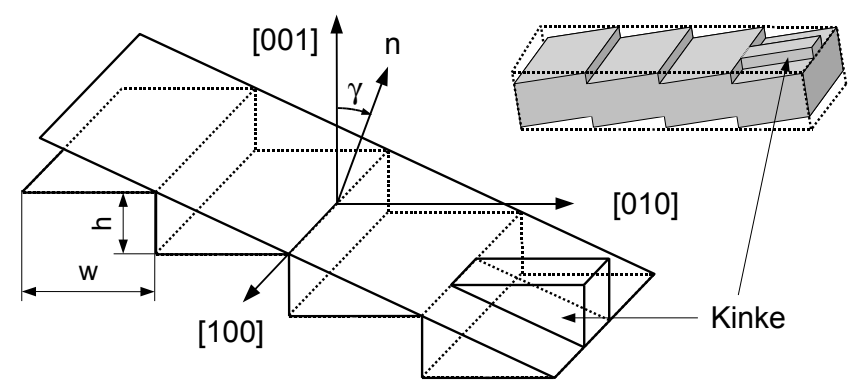

Abbildung A.2: Skizze eines vizinalen Substrates mit Kinke.

$h$ einer STO Einheitszelle sowie die sich aus der Verkippung ergebende Stufenbreite von $w=\frac{h}{\sin (\gamma)}$. Zum Teil sind die Stufenkanten nicht durchgängig gerade, dass heißt sie zeigen sogenannte Kinken. Um eine gleichmäßige Anordnung der Stufen möglichst ohne Kinken zu erhalten, wurden die nochmals gereinigten Substrate vor der Schichtherstellung bei $900{ }^{\circ} \mathrm{C}$ an Luft ausgelagert [32]. Durch die Auslagerung entstehen an der Oberfläche Strontiumoxide [49]. Diese wurden im Ultraschallbad in destilliertem Wasser bei einer Temperatur von $55^{\circ} \mathrm{C}$ für die Dauer von 10 min [68] wieder entfernt.

\section{A.1.4 Strukturierung}

Für die Messungen an den vizinalen Substraten war eine Strukturierung der Filme nötig. Im linken Bild der Abbildung A.3 ist ein PCMO Film auf einem STO

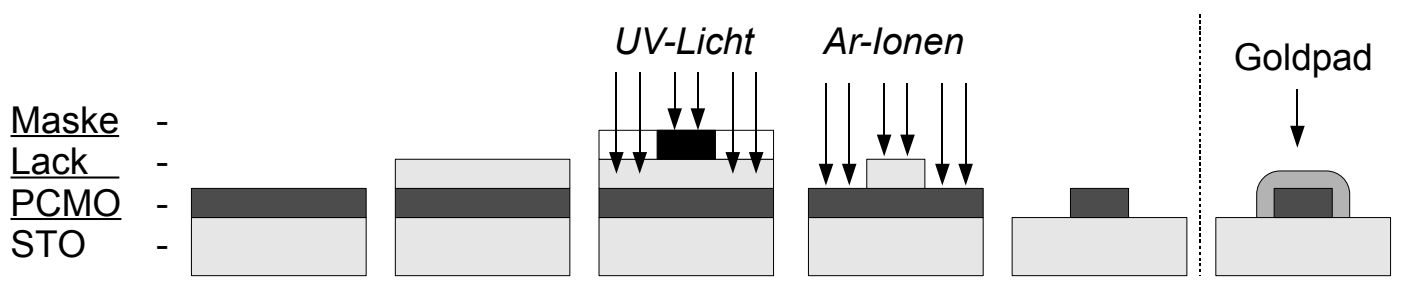

Abbildung A.3: Ablauf der Film-Strukturierung, Goldkontakte für die elektrische Charakterisierung.

Substrat skizziert. Auf diesen wurde photoresistiver Lack AR-P 3510 der Firma Allresist aufgebracht, welcher mittels optischer Lithographie strukturiert wurde. Als Masken für die Strukturierung des Lackes wurden Folien eines Filmbelichters (Firma Santec in Göttingen) verwendet. Die anschließende Strukturierung des 
Films geschah durch einen Sputterätzprozess mit Argon-Ionen. Der Arbeitsdruck betrug dabei 1E-4 mbar. Der Strahlstrom betrug $20 \mathrm{~mA}$ bei einer Beschleunigungsspannung von 700V. Die Ätzrate lag bei 7nm/min. Abschließend wurde im Aceton-Ultraschallbad der Lack wieder entfernt.

\section{A.1.5 Goldkontakte}

Für die elektrische Charakterisierung wurden mittels einer Goldsputteranlage (BIO-RAID Polaron Division) Goldkontakte in verschiedenen Geometrien aufgebracht. Diese sind mit Größenangaben in Abbildung A.4 zu finden. Für planare Substrate war die gesamte Oberfläche des Substrates mit einem PCMO-Dünnfilm bedeckt. Für die vizinalen Substrate musste vor der Kontaktierung der Film strukturiert werden. Die Stegbreiten der Kreuze betrugen nach der Strukturierung bei den Messungen in dieser Arbeit 500 $\mu \mathrm{m}$. Die Masken, durch die das Gold deponiert wurde, sind mittels Funkenerosion aus Aluminium hergestellt wurden.
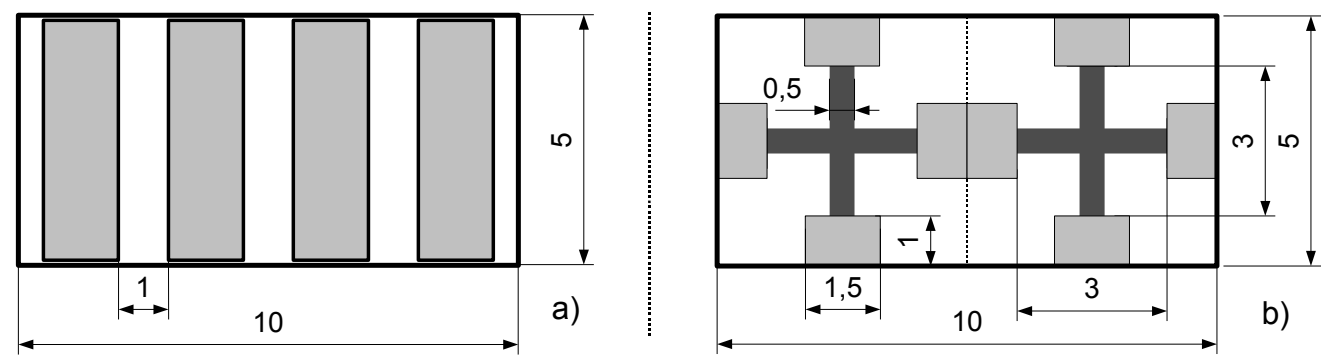

Abbildung A.4: Geometrie der Messkontakte, alle Angaben in mm, a) für planare Substrate, b) für vizinale Substrate.

\section{A.2 Strukturelle Charakterisierung}

\section{A.2.1 Röntgendiffraktometrie}

Für die Messungen in dieser Arbeit wurde ein Vier-Kreis-Diffraktometer X'Pert der Firma Siemens genutzt (siehe Skizze in Abb. A.5 a). Neben dem Messkreis des $2 \Theta$-Winkels kann hier die Probe um drei Achsen (Winkel $\omega, \varphi, \phi$ ) jeweils separat gedreht werden. Der monochromatische Röntgenstrahl der Co-Röhre hatte eine 


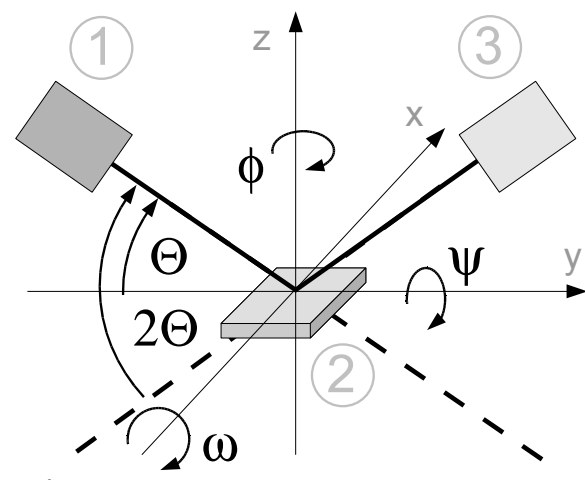

a)

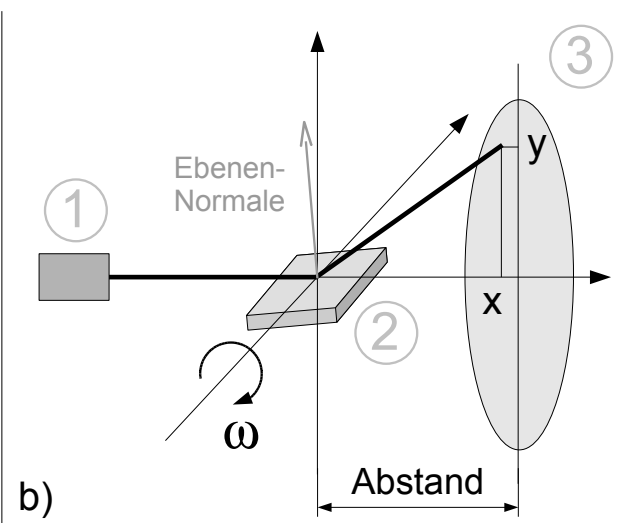

Abbildung A.5: a) 4-Kreis-Diffraktometer mit 0D Detektor b) Diffraktometer mit Flächendetektor; In beiden Skizzen ist 1) die Röntgenquelle, 2) die Probe sowie 3) der Detektor.

Wellenlänge von $\lambda_{C_{o_{K \alpha}}} 0,17902 \mathrm{~nm}$. Die $\Theta-2 \Theta$-Messungen wurden in BraggBrentano-Geometrie durchgeführt. Nach der Bragg-Gleichung ergibt sich aus dem Winkel $\Theta$ der Netzebenenabstand $d$ :

$$
n \lambda_{C o_{K \alpha}}=2 d \sin (\Theta) .
$$

Zudem kann aus der gemessenen Reflexlage die Verspannung $\epsilon$ des Filmes bestimmt werden:

$$
\epsilon=\frac{d_{\text {unverspannt }}-d_{\text {verspannt }}}{d_{\text {unverspannt }}} .
$$

Die Verkippung der (001)-Richtung gegenüber der Oberflächennormale bei vizinalen Substrate wurde durch Rockingkurven bestimmt. Dabei wird für den (200)-Reflex von STO die Beugungsbedingung ( $\Theta \Theta$ Winkel) konstant gehalten und durch Variation des Einfallswinkel $\Theta$ (Drehung der Probe um den Winkel $\omega)$ das Intensitätsmaximum bestimmt. Für die $\Theta-2 \Theta$-Messungen wurde der so bestimmte Verkippwinkel des STO Reflexes als Offset genutzt, was eine Vergleichbarkeit des PCMO Reflexe und somit der Verspannungen ermöglichte.

Eine weitere in dieser Arbeit genutzte Röntgendiffraktometrie basiert auf einem 2D Detektor (siehe Skizze in Abb. A.5 b)). Dies geschah in Zusammenarbeit mit dem Max-Planck-Institut für biophysikalische Chemie in Göttingen. Die genutzte Wellenlänge betrug 0,062 nm. Bei dieser Messmethode wird die Probe in den ansonsten gerade auf die Mitte des Flächendetektor fallenden Röntgenstrahl gebracht. Variable in diesem Messaufbau sind der Probenabstand vom Detektor, 
sowie die Verkippung der Probe um den Winkel $\omega$. Aus der Position der Reflexe auf dem Flächendetektor (in Abb. A.5 b) mit x und y angedeutet), sowie dem Abstand $\left(a_{\overline{P S}}\right)$ der Probe vom Detektor kann man den Winkel $\Theta$ bestimmen:

$$
\Theta=\arctan \left(\frac{\sqrt{x^{2}+y^{2}}}{a_{\overline{P S}}}\right) .
$$

woraus sich wieder mit Formel A.1 der Netzebenenabstand berechnen lässt.

\section{A.2.2 Oberflächenuntersuchung}

Für die Oberflächenuntersuchungen kamen zwei Techniken zum Einsatz, Rasterkraftmikroskopie (Atomic-Force-Microscopy - AFM) und Rasterelektronenmikroskopie (REM). Das AFM war ein NanoScope MultiMode SPM, welches im TappingMode betrieben wurde. Beim REM handelt es sich um ein Philips SEM 515. Die Auflösungsgrenze liegt bei etwa $5 \mathrm{~nm}$, wobei Vergrößerungen von 10 bis 160000 fach möglich sind.

\section{A.2.3 Magnetisierungsmessungen}

Die Magnetisierungsmessungen wurden teilweise in Zusammenarbeit mit der Physikalisch-Technischen Bundesanstalt (PTB) in Braunschweig gemacht. Ein weiterer Teil der Messungen wurde im 1. Physikalischen Institut der Universität Göttingen gemacht. Beide verwendeten SQUID-Magnetometer (Superconducting QUantum Interference Device - Supraleitende Quanteninterferenzeinheit) waren MPMS-5s-Magnetometer der Firma Quantum Design. Die maximal möglichen Magnetfelder für die Magnetesierungsmessungen waren 5T. Die Proben waren so eingebaut, dass das Magnetfeld senkrecht zum Substrat angelegt war.

\section{A.3 Elektronische Charakterisierung}

\section{A.3.1 Messungen im elektrischen Feld}

Für die Messungen im elektrischen Feld in Abhängigkeit von der Temperatur kam ein Kompressionskühler Kryo-Refrigerator RW 2 mit Kaltkopf RGD 210 [88] zum Einsatz. Eine Schemazeichnung des Gifford McMahon Kompressionskühlerkopfes ist in Abbildung A.6 skizziert. Der Temperaturbereich erstreckt sich von 300K bis $35 \mathrm{~K}$. Als Konstantstromquelle wurde ein Keithley 227 CURRENT SOURCE genutzt. Für die Bestimmung der Spannung sowie für die Kontrolle des Stromes kamen K2000 Multimeter der Firma Keithley zum Einsatz. Über das zusätzliche Thermometer konnte die Temperatur in Probennähe bestimmt werden. 


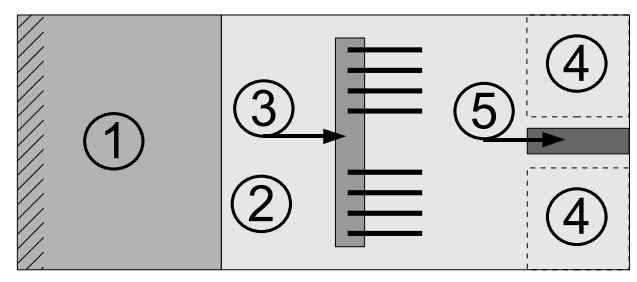

Abbildung A.6: Schemabild des Kühlkopfes, 1) Kühleinheit mit Temperaturreglung, 2) Kühlfinger aus Kupfer, 3) Anschlüsse für elektrische Messungen, bis zu dieser Stelle geschirmte Zuleitungen, 4) Probenposition, 5) Zusätzliches Thermometer in Probennähe.

\section{A.3.2 Messungen im Magnetfeld}

Die elektrische Charakterisierung im Zusammenhang mit einem Magnetfeld erfolgte im physical-properties-measurement-system (im Folgenden kurz PPMS) der Firma Quantum Design. Erweitert wurde das PPMS um ein Elektrometer Keithley 6517A und ein umgebauter multi-function-probe-holder (Multifunktionaler Probenhalter) der Firma Quantum Design (QD-P450A). Die Steuerungssoftware des PPMS MultiVu wurde im Rahmen dieser Arbeit um ein Programm erweitert, was die Schnittstelle zu den zusätzlichen Keithley bot. Damit ist es möglich, synchron die benötigten elektrischen Messdaten sowie die Umgebungsvariablen (Temperatur, Magnetfeld, Position) automatisiert aufzunehmen.

\section{PPMS}

Das PPMS bietet die Möglichkeit in einem Temperaturbereich von 400K bis unter $4 \mathrm{~K}$ zu messen. Das maximal mögliche Magnetfeld beträgt $\pm 9 \mathrm{~T}$. Für alle Messungen in dieser Arbeit wurde der Innenraum auf 4Torr abgepumpt. Neben einem Standartmesskopf bietet das PPMS noch die Möglichkeit mittels eines Probenhalters (Rotator; QD-P310A) zu messen, welcher die Probe im Magnetfeld um eine Achse dreht. Für diese beiden Methoden kamen jedoch nur die integrierten schlecht abgeschirmte Kabel zum Einsatz, was sich als ungeeignet für die Messung hoher Widerstände erwies.

\section{Multi-Funktions-Proben-Halter}

Der Multi-Funktions-Proben-Halter MFPH wurde für die Messung sehr kleiner Ströme angepasst. Für die Zuleitungen wurde das Spezialkabel Kapton Wire (311- 
KAP M-060-COAX) der Firma Allectra verwendet. Dieses ist sehr gut geschirmt sowie für tiefe Temperaturen geeignet. Die Schirmung der Kabel war bis $1 \mathrm{~cm}$ vor der Probe gegeben. Über einen umgebauten Probenhalteraufsatz für den MFPH wurden die Messungen im Magnetfeld senkrecht zum Substrat durchgeführt.

\section{Keithleys}

Möchte man sehr hohe Widerstände messen, so muss man beachten, dass das Spannungsmessgerät parallel zum Widerstand (Probe) geschaltet ist, was einen entsprechend hohen Innenwiderstand des Messgerätes erfordert. Normale Keithley Spannungsmessgeräte, wie zum Beispiel das K2000, haben eine Impedanz von $10^{7} \Omega$. Die untersuchten Proben weisen Widerstände bis $10^{10} \Omega$ auf. Um auch diese messen zu können, wurde ein Elektrometer Keithley K6517A mit einer Impedanz von $2 \cdot 10^{12} \Omega$ eingesetzt. Mit dem K6517A sind nur 2 Punkt Messungen möglich, bei sehr hochohmigen Proben ist der Unterschied zu 4-Punkt Messungen aber vernachlässigbar.

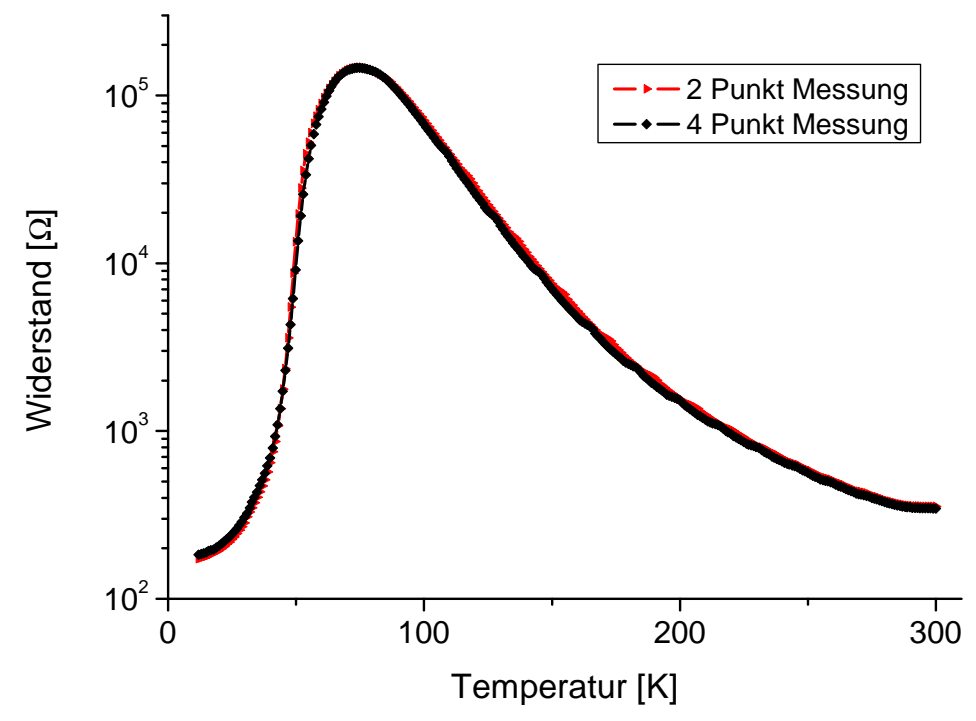

Abbildung A.7: Vergleich einer 4 Punkt sowie einer 2 Punkt Widerstandsmessung.

\section{Messprogramme Erweiterung zu MultiVu}

Das PPMS wird über die Software MultiVu von Quantum Design gesteuert. Das hier kurz vorgestellte Programm dient als Schnittstelle zu den zusätzlichen Keit- 
hleys. In Abbildung A.8 ist ein Screenshot der in Zusammenhang mit dieser Arbeit von mir entwickelten Software zu sehen. Da sowohl das PPMS, wie auch

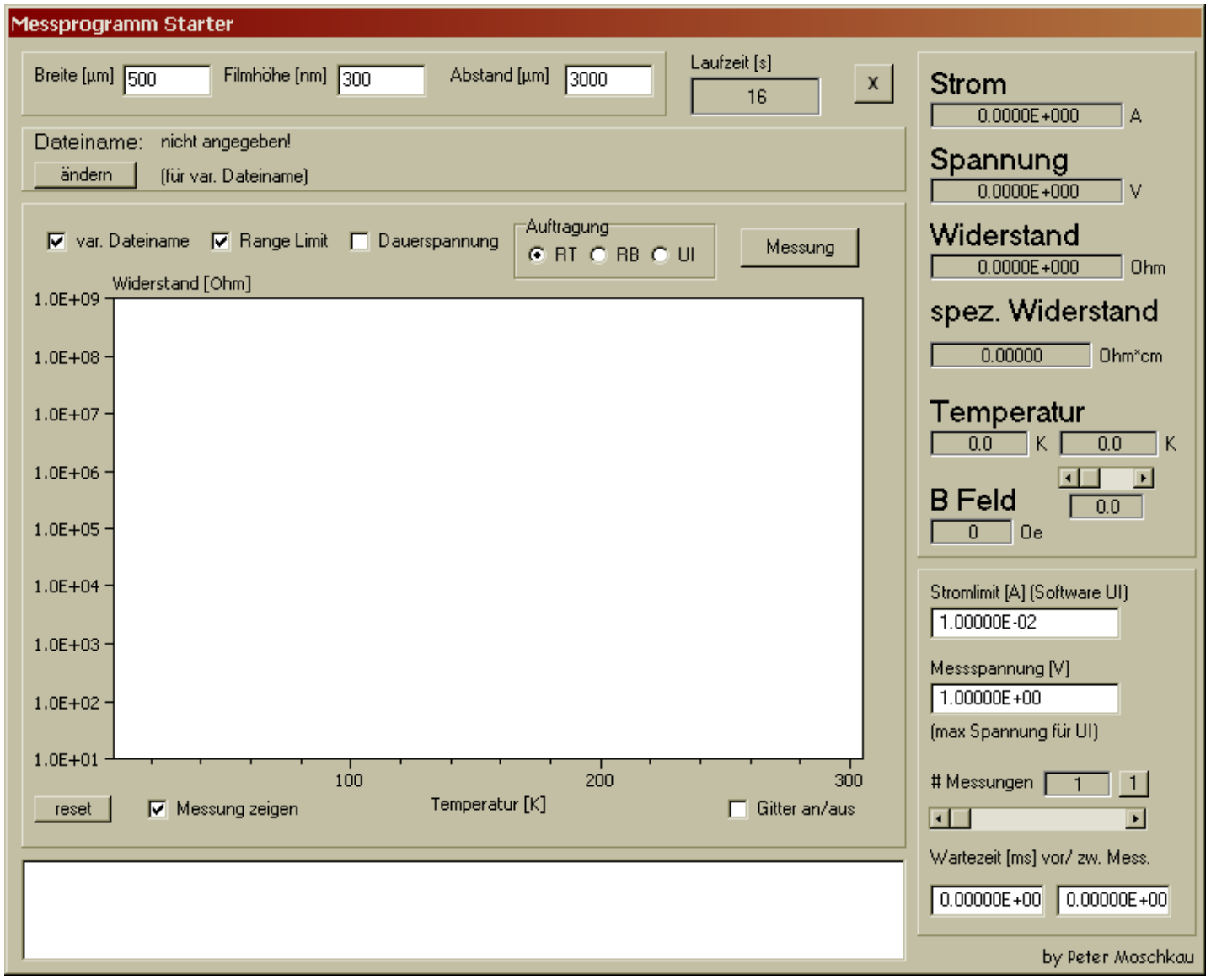

Abbildung A.8: Messprogramm als Erweiterung für MultiVu zur Ansteuerung von zusätzlichen Keithleys.

die Messkeithleys über einen GPIB Port im PC angesprochen wurden, konnte LabView nicht eingesetzt werden, sondern es musste eine eigene Schnittstelle programmiert werden. Das Messprogramm in Abbildung A.8 kann Spannungs-Strom Kennlinien (UI-Kurven), Widerstand über Magnetfeld Kurven (RB-Kuren) sowie Widerstand über Temperatur (RT-Kurven) automatisiert und von der MultiVu Software ferngesteuert aufnehmen. UI-Kurven sowie Messpunkte mit vorgegebener Spannung können auch manuell gemessen werden. Des Weiteren können auch zeitabhängige Messungen mit konstanter Spannung durchgeführt werden. Die Zeit bei angelegter Spannung bis zur Widerstandsmessung kann hierfür variiert werden, sowie die Zeit, die zwischen zwei Messungen liegt. Somit kann die Zeit für Relaxation bestimmt werden, um zum Beispiel Aufwärmeffekte zu 
unterdrücken. Für einen Messpunkt wird für ca. 200 ms Spannung angelegt, insofern die Zeiten im Programm auf $0 \mathrm{~ms}$ gesetzt sind. Länger kann es dauern, wenn es zu einer Messbereichsumschaltung am K6715A kommt. Die Bestimmung des Stromes durch das Keithley erfolgt innerhalb der 200ms nach $100 \mathrm{~ms}$. Der Widerstand wurde aus der vorgegebenen Spannung und dem gemessenen Strom berechnet. Des Weiteren wird ein zusätzliches Keithley K2000 angesteuert, welches den Widerstand eines in direkter Nähe zur Probe angebrachten zusätzlichen Temperatursensor ausliest. Dies gibt die zweite Temperatur im Programm.

\section{A.3.3 Zeitaufgelöste Widerstandsmessungen}

Für die zeitaufgelösten Messungen kam ein Oszillator der Firma Agiland mit 1Ghz Samplingrate zum Einsatz. Hierbei lag der Hauptaugenmerk nicht auf einer absoluten Bestimmung des Widerstandes, sondern auf dem qualitativen zeitliche Verhalten im Messintervall. Der Innenwiderstand des Oszillators $\left(10^{6} \Omega\right)$ wurde für die Messungen bei hohen Widerständen als Shunt genutzt. Als Spannungsquelle für die Messung diente wieder das Keithley K6715A. 


\section{Literaturverzeichnis}

[1] M. Abrashev, V. J. Backstrom, L. Borjesson, M. Pissas, N. Kolev, and M. N. Iliev. Raman spectroscopy of the charge- and orbital-ordered state in lcmo. Phys. Rev. B, 64(14):144429, 2001.

[2] D. N. Argyriou, J. W. Lynn, R. Osborn, B. Campbell, J. F. Mitchell, U. Ruett, H. N. Bordallo, A. Wildes, and C. D. Ling. Glass transition in the polaron dynamics of colossal magnetoresistive manganites. Phys. Rev. Lett., 89(3):036401, Jul 2002.

[3] A. Asamitsu, Y. Tomioka, H. Kuwahara, and Y. Tokura. Current switching of resistive states in magnetoresistive manganites. Nature, 388:3, 1997.

[4] T.L. Aselage, D. Emin, S. S. McCready, E. L. Venturini, M. A. Rodriguez, J. A. Voigt, and T. J. Headley. Metal-semiconductor and magnetic transitions in compensated polycrystalline $\mathrm{LaCaMnO}_{3}$. Phys. Rev. B, 68:134448, 2003.

[5] I.G. Austin and N.F. Mott. Polarons in crystalline and non-crystalline materials. Advances in Physics, 18:71:41-102, 1969.

[6] E.S. Bozin, X. Qiu, M. Schmidt, G. Paglia, J.F. Mitchell, P.G. Radaelli, Th. Proffen, and S.J.L. Billinge. Local structural aspects of the orthorhombic to pseudo-cubic phase transformation in $\mathrm{La}_{1-x} \mathrm{Ca}_{x} \mathrm{MnO}_{3}$. Physica B: Condensed Matter, 385-386(Part 1):110-112, November 2006.

[7] J. Brötz, H. Fuess, T. Haage, J. Zegenhagen, Ch. Jooss, A. Forkl, and R. Warthmann. Anisotropic defect structure and transport properties of YBCO films on vicinal $\mathrm{SrTiO}_{3}(001)$. Journal of Applied Physics, 85(1):635637, 1999.

[8] H. Böttger and V. V. Bryksin. Hopping Conduction in Solids. VCH Verlagsgesellschaft mbH, 1985.

[9] E. Cappelluti and S. Ciuchi. Magnetic and lattice polaron in the holstein t-j model. Phys. Rev. B, 66:165102, 2002. 
[10] J. M. D. Coey. Mixed-valence manganites. Advances in Physics, 48(2):167293, 1999.

[11] J. M. D. Coey, M. Viret, L. Ranno, and K. Ounadjela. Electron localization in mixed-valence manganites. Phys. Rev. Lett., 75(21):3910-3913, 1995.

[12] J. L. Cohn, C. Chiorescu, and J. J. Neumeier. Polaron transport in the paramagnetic phase of electron-doped manganites. Phys. Rev. B, 72:024422, 2005.

[13] C.S.Nelson, J. P. Hill, Doon Gibbs, M. Rajeswari, A. Biswas, S. Shinde, R. L. Greene, T. Venkatesan, A. J. Millis, F. Yokaichiya, C. Giles, D. Casa, C. T. Venkataraman, and T. Gog. Substrate-induced strain effects on $\mathrm{Pr}_{0.6} \mathrm{Ca}_{0.4} \mathrm{MnO}_{3}$ films. J. Phys.: Condens. Matter, 16:13-27, 2004.

[14] Congwu Cui and Trevor A. Tyson. Pressure effects on charge, spin, and metal-insulator transitions in the narrow bandwidth manganite $\mathrm{Pr}_{1-x} \mathrm{Ca}_{x} \mathrm{MnO}_{3}$. Physical Review B (Condensed Matter and Materials Physics), 70(9):094409, 2004.

[15] E Dagotto. Nanoscale Phase Separation and Colossal Magnotoresistance. Springer, Berlin, 1. aufl. edition, 2003.

[16] Pengcheng Dai, J. A. Fernandez-Baca, N. Wakabayashi, E. W. Plummer, Y. Tomioka, and Y. Tokura. Short-Range Polaron Correlations in the Ferromagnetic $L a_{1-x} C a_{x} \mathrm{MnO}_{3}$. Phys. Rev. Lett., 85(12):2553-2556, Sep 2000.

[17] A. Daoud-Aladine, J. Rodríguez-Carvajal, L. Pinsard-Gaudart, M. T. Fernández-Díaz, and A. Revcolevschi. Zener polaron ordering in half-doped manganites. Phys. Rev. Lett., 89(9):097205, 2002.

[18] P. G. de Gennes. Effects of double exchange in magnetic crystals. Phys. Rev., 118(1):141-154, 1960.

[19] V. Dediu, C. Ferdeghini, F. C. Matacotta, P. Nozar, and G. Ruani. Jahnteller dynamics in charge-ordered manganites from raman spectroscopy. Phys. Rev. Lett., 84(19):4489-4492, 2000.

[20] Emin. McGraw Hill Encyclopedia of Science and Technology, volume 14. McGraw, 10 edition, 2007.

[21] D. Emin. Current-driven threshold switching of a small polaron semiconductor to a metastable conductor. Phys. Rev. B, 74:035206, 2006.

[22] David Emin. Optical properties of large and small polarons and bipolarons. Phys. Rev. B, 48(18):13691-13702, Nov 1993. 
[23] D. Feinberg, S.Ciuchi, and F. dePasquale. Squeezing phenomena in interacting electron-phonon systems. Int. J. Mod. Phys. B, 4:1317-1367, 1990.

[24] R. P. Feynman, R. W. Hellwarth, C. K. Iddings, and P. M. Platzman. Mobility of slow electrons in a polar crystal. Phys. Rev., 127(4):1004, 1962.

[25] M. Fiebig, K. Miyano, Y. Tomioka, and Y. Tokura. Visualization of the Local Insulator-Metal Transition in $\mathrm{Pr}_{0.7} \mathrm{Ca}_{0.3} \mathrm{MnO}_{3}$. Science, 280(5371):19251928, 1998.

[26] Masayuki Fujimoto, Hiroshi Koyama, Yuji Nishi, Toshimasa Suzuki, Shinji Kobayashi, Yukio Tamai, and Nobuyoshi Awaya. Crystallographic Domain Structure of an Epitaxial $\left(\mathrm{Pr}_{0.7} \mathrm{Ca}_{0.3}\right) \mathrm{MnO}_{3}$ Thin Film Grown on a SrTiO Single Crystal Substrate. J. Am. Ceram. Soc., 90(7):2205-2209, 2007.

[27] J.L. Garcia-Munoz, M. Fontcuberta Suaaidi, and X Obradors. Bandwidth narrowing in bulk lamno magnetoresistive oxides. J. Phys.: Condens Matter, 8:L787-L793, 1996.

[28] J. B. Goodenough. Magnetism and chemical bond. Interscience Publisher, New York, 1. aufl. edition, 1963.

[29] John B. Goodenough. Theory of the Role of Covalence in the PerovskiteType Manganites [La, M(II)] $\mathrm{MnO}_{3}$. Phys. Rev., 100(2):564-573, 1955.

[30] O.Yu. Gorbenko, I.E. Graboy, A.R. Kaul, and H.W. Zandbergen. Hrem and xrd characterization of epitaxial perovskite manganites. Journal of Magnetism and Magnetic Materials, 211:97-104, 2000.

[31] S. Grenier, J. P. Hill, Doon Gibbs, K. J. Thomas, M. v. Zimmermann, C. S. Nelson, V. Kiryukhin1, Y. Tokura, Y. Tomioka, D. Casa, T. Gog, and C. Venkataraman. Resonant x-ray diffraction of the magnetoresistant perovskite pcmo. Phys. Rev. B, 69(13):134419, 2004.

[32] T. Haage. Untersuchung zu dem Wachstum, der mikroskopischen Struktur und den Transporteigenschaften dünner YBCO-Schichten auf gestufen Substratoberflächen. PhD thesis, Max-Planck-Institut für Festkörperforschung Stuttgart, 1997.

[33] A.-M. Haghiri-Gosnet and J.-P. Renard. Cmr manganites: physics, thin films and devices. Journal of Physics D: Applied Physics, 36(8):R127-R150, 2003.

[34] Ch. Hartinger, F. Mayr, A. Loidl, and T. Kopp. Polaronic excitations in colossal magnetoresistance manganite films. Physical Review B (Condensed Matter and Materials Physics), 73(2):024408, 2006. 
[35] Jiaqing He, Renhui Wang, Jianian Gui, and Cheng Dong. Orthorhombic to Cubic Phase Transition in LCMO Perovskites. Phys. Stat. Sol. B, 229(3):1145-1154, 2002.

[36] M. Hervieu, A. Barnabé, C. Martin, A. Maignan, and B. Raveau. Charge disordering induced by electron irradiation in colossal magnetoresistant manganites. Phys. Rev. B, 60(2):R726-R729, 1999.

[37] J.P. Hill, C.S. Nelson, M. v. Zimmermann, Y.-J.Kim, D.Gibbs, D.Casa, B.Keimer, Y. Murakami, C. Venkataraman, T.Gog, Y.Tomioka, Y. Tokura, V. Kiryukhin, T.Y.Koo, and S.-W.Cheong. Orbital correlations in doped manganites. Applied Physics A, 73:723-730, 2001.

[38] T. Holstein. Studies of polaron motion : Part ii. the small polaron. Annals of Physics, 8:343-389, 1959.

[39] M.F. Hundley, J.J. Neumeier, R.H. Heffner, Q.X. Jia, X.D. Wu, and J.D. Thompson. Interplay between electronic transport and magnetic order in ferromagnetic magnetic manganite thin films. In Spring Meeting - Materials Research Society, 1997.

[40] H. A. Jahn and E. Teller. Stability of Polyatomic Molecules in Degenerate Electronic States. I. Orbital Degeneracy. Royal Society of London Proceedings Series A, 161:220-235, 1937.

[41] Z. Jirak, S. Krupicka, Z. Simsa, M. Dlouha, and S. Vratislav. Neutron diffraction study of $\mathrm{Pr}_{1-\mathrm{x}} \mathrm{Ca}_{\mathrm{x}} \mathrm{MnO}_{3}$ perovskites. Journal of Magnetism and Magnetic Materials, 53:153-166, 1985.

[42] Ch. Jooß, L. Wu, T. Beetz, R. F. Klie, M. Beleggia, M. Schofield, S. Schramm, J. Hoffmann, and Y. Zhu. Polaron melting and ordering as key mechanisms for colossal resistance effects in manganites. PNAS, 104(34):13597-13602, August 2007.

[43] Ch. Jooss, R. Warthmann, and H. Kronmüller. Pinning mechanism of vortices at antiphase boundaries in $\mathrm{YBa}_{2} \mathrm{Cu}_{3} \mathrm{O}_{7-\delta}$. Phys. Rev. B, 61(18):1243312446, May 2000.

[44] S. G. Kaplan, M. Quijada, H. D. Drew, D. B. Tanner, G. C. Xiong, R. Ramesh, C. Kwon, and T. Venkatesan. Optical Evidence for the Dynamic Jahn-Teller Effect in $\mathrm{Nd}_{0.7} \mathrm{Sr}_{0.3} \mathrm{MnO}_{3}$. Phys. Rev. Lett., 77(10):2081-2084, Sep 1996.

[45] Khomskii. Spin Electronics. Springer-Verlag Berlin and Heidelberg GmbH \& Co. K, 2001. 
[46] K. H. Kim, J. H. Jung, D. J. Eom, T. W. Noh, Jaejun Yu, and E. J. Choi. Scaling behavior of spectral weight changes in perovskite manganites lcmo. Phys. Rev. Lett., 81(22):4983-4986, Nov 1998.

[47] V. Kiryukhin, D. Casa, J. P. Hill, B. Keimer, A. Vigliante, Y. Tomioka, and Y. Tokura. An X-ray-induced insulator-metal transition in a magnetoresistive manganite. Nature, 386:813-815, 1997.

[48] K. Knizek, J. Hejtmanek, , and Z. Jirak. Structure, Magnetism, and Transport Properties of $\mathrm{Pr}_{1-x} \mathrm{Sr}_{x} \mathrm{MnO}_{3}$ (x=0.45-0.75) up to $1200 \mathrm{~K}$. Chem. Mater., 16:1104-1110, 2004.

[49] D. Kobayashi, R. Hashimoto, and A. CHIKAMATSU. Sr surface segregation and water cleaning for atomically controlled sto (001) substrates studied by photoemission spectroscopy. Journal of electron spectroscopy and related phenomena, 144:443-446, 2005.

[50] I. Komissarov, Y. Zhang, G. J. Nieuwenhuys, E. Morenzoni, T. Prokscha, A. Suter, and J. Aarts. Investigating the occurrence of magnetic order in strained thin films of $\mathrm{Pr}_{0.5} \mathrm{Ca}_{0.5} \mathrm{MnO}_{3}$ by muon spin relaxation. EPL (Europhysics Letters), 83(4):47013 (6pp), 2008.

[51] H.-U. Krebs and O. Bremert. Pulsed laser deposition of thin metallic alloys. Applied Physics Letter, 62:2341, 1993.

[52] K. I. Kugel and D. I. Khomskii. The jahn-teller effect and magnetism: transition metal compounds. Sov. Phys. Usp., 25(231):231-256, 1982.

[53] L. G. Lang and Yu. A. Firsov. Mobility of small polarons at low temperatures. Soviet Physics - solid states, 5(10):2049-2060, 1964.

[54] H. J. Lee, J. H. Jung, Y. S. Lee, J. S. Ahn, T. W. Noh, K. H. Kim, and S-W. Cheong. Optical properties of a $\mathrm{Nd}_{0.7} \mathrm{Sr}_{0.3} \mathrm{MnO}_{3}$ single crystal. Phys. Rev. B, 60(8):5251-5257, Aug 1999.

[55] Y. S. Lee, T. Arima, S. Onoda, Y. Okimoto, Y. Tokunaga, R. Mathieu, X. Z. Yu, J. P. He, Y. Kaneko, Y. Matsui, N. Nagaosa, and Y. Tokura. Correlation of electronic structure and ordered charge and orbital patterns for singlelayered manganites in a wide hole-doping range $(0<=\mathrm{x}<=1)$. Physical Review B (Condensed Matter and Materials Physics), 75(14):144407, 2007.

[56] M. R. Lees, J. Barratt, C. D. Dewhurst, G. Balakrishnan, C. V. Tomy, and D. McK. Paul. Field induced insulator-metal transition in $\operatorname{Pr}_{1-\mathrm{x}} \mathrm{Ca}_{\mathrm{x}} \mathrm{MnO}_{3}$. Physica B: Condensed Matter, 223-224:532 - 534, 1996. Proceedings of the International Conference on Strongly Correlated Electron Systems. 
[57] A. Levstik, C. FilipiC, V. Bobnar, A. PotoCnik, D. ArCon, S. Drnovsek, J. Holc, and Z. JagliCic. Ordering of polarons in the charge-disordered phase of $\mathrm{Pr}_{0.7} \mathrm{Ca}_{0.3} \mathrm{MnO}_{3}$. Physical Review $B$ (Condensed Matter and Materials Physics), 79(15):153110, 2009.

[58] J. W. Lynn, D. N. Argyriou, Y. Ren, Y. Chen, Y. M. Mukovskii, and D. A. Shulyatev. Order and dynamics of intrinsic nanoscale inhomogeneities in manganites. Physical Review B (Condensed Matter and Materials Physics), 76(1):014437, 2007.

[59] A. Machida, Y. Moritomo, and A. Nakamura. Spectroscopic evidence for formation of small polarons in doped manganites. Phys. Rev. B, 58(8):R4281-R4284, Aug 1998.

[60] F. Mayr, C. Hartinger, M. Paraskevopoulos, A. Pimenov, J. Hemberger, A. Loidl, A. A. Mukhin, and A. M. Balbashov. High-frequency conductivity and phonon properties of $\mathrm{La}_{7 / 8} \mathrm{Sr}_{1 / 8} \mathrm{MnO}_{3}$. Phys. Rev. B, 62(23):1567315679, Dec 2000.

[61] A. J. Millis. Lattice effects in magnetoresistive manganese perovskites. Nature, 392(6672):147-150, March 1998.

[62] A. J. Millis, P. B. Littlewood, and B. I. Shraiman. Double Exchange Alone Does Not Explain the Resistivity of $\mathrm{La}_{1-\mathrm{x}} \mathrm{Sr}_{\mathrm{x}} \mathrm{MnO}_{3}$. Phys. Rev. Lett., 74(25):5144-5147, 1995.

[63] K. Miyano, T. Tanaka, Y. Tomioka, and Y. Tokura. Photoinduced insulator-to-metal transition in a perovskite manganite. Phys. Rev. Lett., 78(22):4257-4260, 1997.

[64] A. Morimoto and T. Shimizu. Handbook Of Thin Film Process Technology. Institute Of Physics, 1995.

[65] Y. Moritomo, A. Machida, K. Matsuda, M. Ichida, and A. Nakamura. Magnetization-dependent behaviors of interband transitions between the exchange-split bands in doped manganite films. Phys. Rev. B, 56(9):50885091, Sep 1997.

[66] P. Moschkau, S. Schramm, J. Hoffmann, J. Fladerer, Ch. Jooss, L. Wu, and Y. Zhu. Electronic phase separation and the CMR effect in $\operatorname{Pr}_{1-x} \mathrm{Ca}_{x} \mathrm{MnO}_{3}$ films on (0 01 1) vicinal $\mathrm{SrTiO}_{3}$ substrates. Materials Science and Engineering: $B, 144(1-3): 78$ - 82, 2007. EMRS 2007, Symposium H: Nanoscale Tailoring of Defect Structures for Optimized Functional and Multifunctional Oxide Films. 
[67] X. Obradors, L. M. Paulius, M. B. Maple, J. B. Torrance, A. I. Nazzal, J. Fontcuberta, and X. Granados. Pressure dependence of the metal-insulator transition in the charge-transfer oxides. Phys. Rev. B, 47(18):12353-12356, May 1993.

[68] T. Ohnishi, K. Shibuya, M. Lippmaa, D. Kobayashi, H. Kumigashira, M. Oshima, and H. Koinuma. Preparation of thermally stable tioterminated sto (100) substrate surfaces. Applied Physics Letter, 85(2):272, 2004.

[69] Y. Okimoto, T. Katsufuji, T. Ishikawa, A. Urushibara, T. Arima, and Y. Tokura. Anomalous variation of optical spectra with spin polarization in double-exchange ferromagnet: la1-xsrxmno3. Phys. Rev. Lett., 75(1):109-112, Jul 1995.

[70] Y. Okimoto, Y. Tomioka, Y. Onose, Y. Otsuka, and Y. Tokura. Optical study of pcmo $(\mathrm{x}=0.4)$ in a magnetic field: Variation of electronic structure with charge ordering and disordering phase transitions. Phys. Rev. B, 59(11):7401, 1999.

[71] S. Parashar, E. E. Ebenso, A. R. Raju, and C. N. R. Rao. Insulator-metal transitions induced by electric and magnetic fields, in thin films of chargeordered $\mathrm{Pr}_{1-\mathrm{x}} \mathrm{Ca}_{\mathrm{x}} \mathrm{MnO}_{3}$. Solid State Communications, 114:295-199, 2000.

[72] V. Ponnambalam, S. Parashar, A. R. Raju, and C. N. R. Rao. Electricfield-induced insulator metal transitions in thin films of charge-ordered rareearth manganates. Applied Physics Letters, 74(2):3, 1999.

[73] W. Prellier, A. M. Haghiri-Gosnet, B. Mercey, Ph. Lecoeur, M. Hervieu, Ch. Simon, and B. Raveau. Spectacular decrease of the melting magnetic field in the charge-ordered state of $\operatorname{Pr}_{0.5} \mathrm{Ca}_{0.5} \mathrm{MnO}_{3}$ films under tensile strain. Applied Physics Letter, 77(7):1023, 2000.

[74] W. Prellier, Ch. Simon, A. M. Haghiri-Gosnet, B. Mercey, and B. Raveau. Thickness dependence of the stability of the charge-ordered state in $\mathrm{Pr}_{0.5} \mathrm{Ca}_{0.5} \mathrm{MnO}_{3}$ thin films. Phys. Rev. B, 62(24), 2000.

[75] W. Prellier, Ch. Simon, B. Mercey, M. Hervieu, A. M. Haghiri-Gosnet, D. Saurel, Ph. Lecoeur, and B. Raveau. Stress-induced metallic behavior under magnetic field in PCMO ( $\mathrm{x}=0.5$ and 0.4$)$ thin films (invited). $J$. Appl. Phys., 89:6612, 2001.

[76] V. G. Prokhorov, G. G. Kaminsky, V. S. Flis, and Young Pak Lee. Temperature dependence of resistance of $\operatorname{Pr}_{0.65} \mathrm{Ca}_{0.35} \mathrm{MnO}_{3}$ films prepared by pulsed laser deposition. Low Temperature Physics, 25(10):792-796, 1999. 
[77] Y. H. Ren, H. B. Zhao, G. Lüpke, C. S. Hong, N. H. Hur, Y. F. Hu, and Q. Li. Ultrafast photoinduced reflectivity transients in doped manganite. J Chem Phys, 121(1):436-40, 2004.

[78] O.F. Schirmer. persönliche Mitteilung von Hr. O.F. Schirmer.

[79] S. Schramm. Transport und Ordnung von Polaronen im Manganatsystem mit kolossalem Elektrowiderstand. PhD thesis, Materialphysik Universität Göttingen, 2006.

[80] S. Schramm, J. Hoffmann, and Ch. Jooss. Transport and ordering of polarons in cer manganites prcamno. J. Phys.: Condens. Matter, 20:395231, 2008.

[81] S. K. Singh, S. B. Palmer, D. McK. Paul, and M. R. Lees. Growth, transport, and magnetic properties of $\mathrm{Pr}_{0.67} \mathrm{Ca}_{0.33} \mathrm{MnO}_{3}$ thin films. Applied Physics Letters, 69(2):263-265, 1996.

[82] N. Takubo, I. Onishi, K. Takubo, T. Mizokawa, and K. Miyano. Photoinduced metal-to-insulator transition in a manganite thin film. Phys. Rev. Lett., 101(17):177403, 2008.

[83] Y. Tomioka, A. Asamitsu, H. Kuwahara, Y. Moritomo, and Y. Tokura. Magnetic-field-induced metal-insulator phenomena in $\mathrm{Pr}_{1-\mathrm{x}} \mathrm{Ca}_{\mathrm{x}} \mathrm{MnO}_{3}$ with controlled charge-ordering instability. Phys. Rev. B, 53(4):R1689-R1692, 1996.

[84] Y. Tomioka and Y. Tokura. Bicritical features of the metal-insulator transition in bandwidth-controlled manganites: Single crystals of pcsmo. PRB, 66:104416, 2002.

[85] B. B. van Aken, T. T. M. Palstra, A. Filippetti, and N. A. Spaldin. The origin of ferroelectricity in magnetoelectric ymno. Nature Materials, 3:164170, 2004.

[86] M. Viret. Transport properties of mixed-valence manganites. In M. J. Thornton and M. Ziese, editors, Spin Electronics, chapter 6, pages 117158. Springer, Berlin, 1. aufl. edition, 2001.

[87] W. Westhäuser, S. Schramm, J. Hofmann, and Ch. Jooss. Comparative study of magnetic and electric field induced insulator-metal-transitions in pcmo films. European Physical Journal B, 53:323-331, 2006.

[88] Wilko Westhäuser. Transporteigenschaften von Manganat-Filmen in magnetischen und elektrischen Feldern am Beispiel von $\mathrm{Pr}_{0,68} \mathrm{Ca}_{0,32} \mathrm{MnO}_{3}$. Diplomarbeit, Institut für Materialphysik, Universität Göttingen, 2004. 
[89] P. R. Willmot and J.R. Huber. Pulsed laser vaporization and deposition. Rev. Mod. Phys., 72:315 - 328, 2000.

[90] H. Windischmann. Intrinsic stress in sputter-deposited thin films. Criticul Reviews in Solid State und Materials Sciences, 17(6):547-596, 1992.

[91] Hyungje Woo, Trevor A Tyson, Mark Croft, and Sang-Wook Cheong. Evidence for spin clusters and glassy behaviour in $\mathrm{Bi}_{1-\mathrm{x}} \mathrm{Ca}_{\mathrm{x}} \mathrm{MnO}_{3}$ (x 0,875). Journal of Physics: Condensed Matter, 16(15):2689-2705, 2004.

[92] P. M. Woodward. Octahedral Tilting in Perovskites. II. Structure Stabilizing Forces. Acta Crystallographica Section B, 53(1):44-66, 1997.

[93] L. Wu, R. F. Klie, Y. Zhu, and Ch. Jooss. Experimental confermation of zener-polaron-type charge and orbital ordering in pcmo. Phys. Rev. B, $76: 174210,2007$.

[94] Z. Q. Yang, Y. Q. Zhang, J. Aarts, M.-Y. Wu, and H. W. Zandbergen. Enhancing the charge ordering temperature in thin films of $\operatorname{Pr}_{0.5} \mathrm{Ca}_{0.5} \mathrm{MnO}_{3}$ by strain. Applied Physics Letter, 88:072507, 2006.

[95] S. Yoon, H. L. Liu, G. Schollerer, S. L. Cooper, P. D. Han, D. A. Payne, S.-W. Cheong, and Z. Fisk. Raman and optical spectroscopic studies of small-to-large polaron crossover in the perovskite manganese oxides. Phys. Rev. B, 58(5):2795-2801, Aug 1998.

[96] H. Yoshizawa, R. Kajimoto, H. Kawano, Y. Tomioka, and Y. Tokura. Bandwidth-control-induced insulator-metal transition in $\mathrm{Pr}_{0.65}\left(\mathrm{Ca}_{1-\mathrm{y}} \mathrm{Sr}_{\mathrm{y}}\right)_{0.35} \mathrm{MnO}_{3}$ and $\mathrm{Pr}_{0.7} \mathrm{Ca}_{0.3} \mathrm{MnO}_{3}$. Phys. Rev. B, 55(5):27292732, 1997.

[97] H. Yoshizawa, H. Kawano, Y. Tomioka, and Y. Tokura. Neutrondiffraction study of the magnetic-field-induced metal-insulator transition in $\mathrm{Pr}_{0.7} \mathrm{Ca}_{0.3} \mathrm{MnO}_{3}$. Phys. Rev. B, 52(18):R13145-R13148, Nov 1995.

[98] H. w. Zandbergen, J. Jansen, S. Freisem, T. Nojimats, , and J. Aartst. Atomic structure and microstructure of very thin films of $\mathrm{La}_{0.73} \mathrm{Ca}_{0.27} \mathrm{MnO}_{3}$ on $\mathrm{SrTi0}_{3}$. Phil. Mag. A, 80:337-350, 2000.

[99] C. Zener. Interaction between the $d$-Shells in the Transition Metals. II. Ferromagnetic Compounds of Manganese with Perovskite Structure. Phys. Rev., 82(3):403-405, 1951.

[100] G. Zhao, H. Keller, and W. Prellier. Unusual electrical transport mechanism in the ferromagnetic state of the magnetoresistive manganites. J. Phys.: Condens. Matter, 12:L361-L366, 2000. 
[101] M. v. Zimmermann, C. S. Nelson, J. P. Hill, Doon Gibbs, M. Blume, D. Casa, B. Keimer, Y. Murakami, C.-C. Kao, C. Venkataraman, T. Gog, Y. Tomioka, and Y. Tokura. X-ray resonant scattering studies of orbital and charge ordering in $\mathrm{Pr}_{1-\mathrm{x}} \mathrm{Ca}_{\mathrm{x}} \mathrm{MnO}_{3}$. Phys. Rev. B, 64(19):195133, 2001.

[102] Liang-Jian Zou, H. Q. Lin, and Q.-Q. Zheng. Composite polaron mechanism for colossal magnetoresistance in perovskite manganites. Journal of Applied Physics, 83(11):7363-7365, 1998. 


\section{Danksagung}

Ich bedanke mich herzlich bei allen, die zum Entstehen und Gelingen dieser Arbeit beigetragen haben. Insbesondere bei

Herrn Prof. Dr. Christian Jooß für die Vergabe der Arbeit,

Herrn PD Dr. Vasily Moshnyaga für die Übernahme des Korreferates,

Dr. Jörg Hoffmann für seine hilfreiche Unterstützung und wertvollen Ratschläge, den Technikern der Materialphysik für die technische Unterstützung, meinen Arbeitsgruppenkollegen für die kollegiale Zusammenarbeit,

Kathrin Bornemann, Sven Schnittger, Simon Reich und Alexandra Lüttich für die Durchsicht des Manuskripts,

Anna Hartje für ein familiäres Zuhause während dieser Arbeit

und meinen Eltern und Großeltern für ihre lebenslange Unterstützung in allen Bereichen. 


\section{Lebenslauf}

\section{Persönliche Daten}

Name:

Vorname:

Geburtsdatum:

Geburtsort:

Nationalität:

Familienstand:

\section{Schulbildung}

09/83 - 07/88

$09 / 88-07 / 93$

08/93-07/96

Ersatzdienst

08/96 - 09/97

\section{Ausbildung}

$11 / 97$

04/01

$11 / 04$

$01 / 05$
Moschkau

Peter

01. 07.1976

Bad Salzungen

deutsch

ledig

POS Wilhelm Pick (Bad Salzungen)

Realschule Erich Mäder (Eisenach)

Berufliches Gymnasium Ludwig Erhard (Seebach)

ASB Rettungsdienst Eisenach

Begin des Studiums der Physik an der

Georg-August-Universität Göttingen

Diplom-Vorprüfung in Physik

Abschluss als Diplom-Physiker

Begin der Promotion an der

Georg-August-Universität Göttingen 